\title{
BLOW UP FOR THE CRITICAL GKDV EQUATION. II: MINIMAL MASS DYNAMICS
}

\author{
YVAN MARTEL, FRANK MERLE, AND PIERRE RAPHAËL
}

\begin{abstract}
We consider the mass critical (gKdV) equation $u_{t}+\left(u_{x x}+u^{5}\right)_{x}=0$ for initial data in $H^{1}$. We first prove the existence and uniqueness in the energy space of a minimal mass blow up solution and give a sharp description of the corresponding blow up soliton-like bubble. We then show that this solution is the universal attractor of all solutions near the ground state which have a defocusing behavior. This allows us to sharpen the description of near soliton dynamics obtained in 33.
\end{abstract}

\section{Introduction}

1.1. Setting of the problem. We continue the study of the mass critical generalized Korteweg-de Vries equation:

$$
(\operatorname{gKdV}) \begin{cases}u_{t}+\left(u_{x x}+u^{5}\right)_{x}=0, & (t, x) \in[0, T) \times \mathbb{R}, \\ u(0, x)=u_{0}(x), & x \in \mathbb{R},\end{cases}
$$

initiated in Part I [33]. The Cauchy problem is locally well posed in the energy space $H^{1}$ from Kenig, Ponce and Vega [15, 16], and given $u_{0} \in H^{1}$, there exists a unique 1 maximal solution $u(t)$ of (1.1) in $C\left([0, T), H^{1}\right)$ and

$$
T<+\infty \text { implies } \lim _{t \rightarrow T}\left\|u_{x}(t)\right\|_{L^{2}}=+\infty .
$$

The Cauchy problem for (1.1) is also locally well-posed in $L^{2}$ and given $u_{0} \in L^{2}$, there exists a unique maximal solution $u(t)$ of (1.1) in $C\left([0, T), L^{2}\right)$ with either $T=+\infty$ or

$$
T<+\infty \text { and then }\|u\|_{L_{x}^{5} L_{(0, T)}^{10}}=\infty .
$$

Moreover, $H^{1}$ solutions satisfy the conservation of mass and energy:

$$
M(u(t))=\int u^{2}(t)=M_{0}, \quad E(u(t))=\frac{1}{2} \int u_{x}^{2}(t)-\frac{1}{6} \int u^{6}(t)=E_{0} .
$$

The symmetry group of (1.1) is continuous in $H^{1}$ and given by

$$
\epsilon_{0} \lambda_{0}^{\frac{1}{2}} u\left(\lambda_{0}^{3}\left(t-t_{0}\right), \lambda_{0}\left(x-x_{0}\right)\right), \quad\left(\epsilon_{0}, \lambda_{0}, x_{0}, t_{0}\right) \in\{-1,1\} \times \mathbb{R}_{+}^{*} \times \mathbb{R} \times \mathbb{R} .
$$

In particular the scaling symmetry leaves the $L^{2}$ norm invariant and hence the problem is mass or $L^{2}$ critical.

Travelling wave solutions play a distinguished role in the analysis

$$
u(t, x)=Q(x-t)
$$

where $Q$ is the ground state solitary wave

$$
Q(x)=\left(\frac{3}{\cosh ^{2}(2 x)}\right)^{\frac{1}{4}}
$$

\footnotetext{
$1_{\text {in a certain sense }}$
} 
which attains the sharp Gagliardo-Nirenberg inequality, [55]:

$$
\forall v \in H^{1}, \quad \int|v|^{6} \leq \int v_{x}^{2}\left(\frac{\int v^{2}}{\int Q^{2}}\right)^{2} .
$$

The conservation of mass and energy and the blow up criterion (1.2) ensure that $H^{1}$ initial data with subcritical mass $\left\|u_{0}\right\|_{L^{2}}<\|Q\|_{L^{2}}$ generate global in time solutions.

1.2. The flow near the ground state. In the series of works [26, 27, 39, 28, 29], Martel and Merle obtain the first qualitative information on the flow for small super critical mass initial data $\|Q\|_{L^{2}}<\left\|u_{0}\right\|_{L^{2}}<\|Q\|_{L^{2}}+\alpha^{*}, 0<\alpha^{*} \ll 1$, in particular the existence of finite time blow up solutions for $E_{0}<0$ and the classification of $Q$ as the unique global attractor of all $H^{1}$ blow up solutions.

In Part I [33], we have revisited the blow up analysis in light of recent developments related to blow up for the mass critical Schrödinger equation [40, 41, 42, 43, [44, 45] and energy critical geometrical equations [51, 46, 52].

More precisely, let the set of initial data

$$
\mathcal{A}=\left\{u_{0}=Q+\varepsilon_{0} \text { with }\left\|\varepsilon_{0}\right\|_{H^{1}}<\alpha_{0} \text { and } \int_{y>0} y^{10} \varepsilon_{0}^{2}<1\right\},
$$

and consider the $L^{2}$ tube around the family of solitary waves

$$
\mathcal{T}_{\alpha^{*}}=\left\{u \in H^{1} \quad \text { with } \inf _{\lambda_{0}>0, x_{0} \in \mathbb{R}}\left\|u-\frac{1}{\lambda_{0}^{\frac{1}{2}}} Q\left(\frac{.-x_{0}}{\lambda_{0}}\right)\right\|_{L^{2}}<\alpha^{*}\right\} .
$$

In [33], we have proved the following (see Theorems 1.1 and 1.2 in [33] for more details).

Theorem 1.1 (Rigidity of the flow in $\mathcal{A}$, 33]). Let $0<\alpha_{0} \ll \alpha^{*} \ll 1$ and $u_{0} \in \mathcal{A}$. Let $u \in \mathcal{C}\left([0, T), H^{1}\right)$ be the corresponding solution to (1.1). Then, one of the following three scenarios occurs:

(Blow up): the solution blows up in finite time $0<T<+\infty$ in the universal regime

$$
\|u(t)\|_{H^{1}}=\frac{\ell\left(u_{0}\right)+o(1)}{T-t} \text { as } t \rightarrow T, \quad \ell\left(u_{0}\right)>0 .
$$

(Soliton): the solution is global $T=+\infty$ and converges asymptotically to a solitary wave.

(Exit): the solution leaves the tube $\mathcal{T}_{\alpha^{*}}$ at some time $0<t^{*}<+\infty$.

Moreover, the scenarios (Blow up) and (Exit) are stable by small perturbation of the data in $\mathcal{A}$.

Our aim in this paper is first to classify the minimal mass dynamics $\left\|u_{0}\right\|_{L^{2}}=$ $\|Q\|_{L^{2}}$ and then, from this classification, to complete the description obtained in Theorem 1.1 in the (Exit) regime. Indeed, we will show that for $\alpha_{0}$ small enough, the (Exit) case is directly connected to the understanding of minimal mass dynamics.

1.3. Minimal mass dynamics. The question of existence and possibly uniqueness of minimal blow up dynamics for dispersive and parabolic PDE's has motivated several works since the pioneering result by Merle [37] for the mass critical nonlinear Schrödinger equation:

$$
(\mathrm{NLS}) \quad \mathrm{i} \partial_{t} u+\Delta u+|u|^{\frac{4}{N}} u=0, \quad(t, x) \in \mathbb{R} \times \mathbb{R}^{N} .
$$


Let us recall that for (NLS), the pseudo conformal symmetry generates an explicit minimal mass blow up solution

$$
S_{\mathrm{NLS}}(t, x)=\frac{1}{t^{N / 2}} e^{-i \frac{|x|^{2}}{4 t}-\frac{i}{t}} Q_{\mathrm{NLS}}\left(\frac{x}{t}\right)
$$

where $Q_{\text {NLS }}$ is the ground state solution to

$$
\Delta Q_{\mathrm{NLS}}-Q_{\mathrm{NLS}}+Q_{\mathrm{NLS}}^{1+\frac{4}{N}}=0, \quad Q>0, \quad Q \in H^{1} .
$$

Merle proved in 37] that $S_{\mathrm{NLS}}$ is the unique (up to the symmetries of the equation) minimal mass blow up element in the energy space. The proof heavily relies on the pseudo conformal symmetry. Such minimal blow up dynamics have also been exhibited for the energy critical NLS and wave problems [11, [10, using the virial algebra and a fixed point argument. For the inhomogeneous mass critical (NLS) in dimension 2 :

$$
\mathrm{i} \partial_{t} u+\Delta u+k(x)|u|^{2} u=0,
$$

while Merle [38] derived sufficient conditions on $k(x)$ to ensure the nonexistence of minimal elements, Raphä̈l and Szeftel [53] introduced a more dynamical approach to existence and uniqueness under a necessary and sufficient condition on $k(x)$. A robust energy method is implemented to completely classify the minimal mass blow up, in regimes such that the inhomogeneity $k$ influences dramatically the bubble of concentration (1.6) - in contrast with direct perturbative methods developed in [3], [4], 1], see also 20] for existence in the one dimensional half wave problem.

Recall that for the mass critical (gKdV) problem (1.1), Martel and Merle [30] obtained the following global existence result for minimal mass solutions with decay on the right.

Theorem 1.2 (Global existence at minimal mass, [30]). Let $u_{0} \in H^{1}$ with $\left\|u_{0}\right\|_{L^{2}}=$ $\|Q\|_{L^{2}}$ and

$$
\sup _{x_{0}>0} x_{0}^{3} \int_{x>x_{0}} u_{0}^{2}(x) d x<+\infty .
$$

Then, the corresponding solution $u(t)$ of (1.1) is global for $t>0$.

In other words, minimal mass blow up is not compatible with the decay (1.7). This is in agreement with the analysis in [33] where the threshold dynamics for data in $\mathcal{A}$ between the stable (Blow up) and (Exit) regimes is proved to correspond to a solitary wave behavior - and not to a minimal blow up. We refer to [45] for a further discussion of threshold dynamics.

1.4. Statement of the result. The first main result of this paper is the existence and uniqueness in the energy space of a minimal mass blow up element:

Theorem 1.3 (Existence and uniqueness of the minimal mass blow up element).

(i) Existence. There exists a solution $S(t) \in \mathcal{C}\left((0,+\infty), H^{1}\right)$ to (1.1) with minimal mass $\|S(t)\|_{L^{2}}=\|Q\|_{L^{2}}$ which blows up backward at the origin:

$$
S(t, x)-\frac{1}{t^{\frac{1}{2}}} Q\left(\frac{x+\frac{1}{t}+\bar{c} t}{t}\right) \rightarrow 0 \text { in } L^{2} \text { as } t \downarrow 0
$$

at the speed

$$
\|S(t)\|_{H^{1}} \sim \frac{C^{*}}{t} \quad \text { as } t \downarrow 0
$$

for some universal constants $\bar{c}, C^{*}$. Moreover, $S$ is smooth and well localized to the right in space:

$$
\forall x \geq 1, \quad S(1, x) \leq e^{-C x} .
$$


(ii) Uniqueness. Let $u_{0} \in H^{1}$ with $\left\|u_{0}\right\|_{L^{2}}=\|Q\|_{L^{2}}$ and assume that the corresponding solution $u(t)$ to (1.1) blows up in finite time. Then

$$
u \equiv S
$$

up to the symmetries of the flow.

Observe that the minimal element blows up with speed (1.8) which is the same as in the (Blow up) regime obtained in Theorem 1.1. However, the case of (Blow up) in Theorem 1.1 is shown to be stable by small perturbation in $\mathcal{A}$, while minimal mass blow up is unstable by perturbation of the data $S(0) \rightarrow(1-\varepsilon) S(0), \varepsilon>0$, since the corresponding solution has subcritical mass and is thus global in time. This shows that the decay assumption to the right in Theorem 1.1 is essential and that the minimal blow up solution has slow decay to the left? 2 . The nature of the minimal blow up is different from the one of stable blow up.

We now relate the (Exit) case in Theorem 1.1 to the minimal mass blow up dynamics. We claim that at the (Exit) time, the solution is $L^{2}$ close up to renormalization to the unique minimal solution $S(t)$.

Theorem 1.4 (Description of the (Exit) scenario). Let $u(t, x)$ be a solution of (1.1) corresponding to the (Exit) scenario in Theorem 1.1 and let $t_{u}^{*} \gg 1$ be the corresponding exit time. Then there exist $\sigma^{*}=\sigma^{*}\left(\alpha^{*}\right)$ (independent of $u$ ) and $\left(\lambda_{u}^{*}, x_{u}^{*}\right)$ such that

$$
\left\|\left(\lambda_{u}^{*}\right)^{\frac{1}{2}} u\left(t_{u}^{*}, \lambda_{u}^{*} x+x_{u}^{*}\right)-S\left(\sigma^{*}, x\right)\right\|_{L^{2}} \leq \delta_{I}\left(\alpha_{0}\right),
$$

where $\delta_{I}\left(\alpha_{0}\right) \rightarrow 0$ as $\alpha_{0} \rightarrow 0$.

Note that uniqueness in Theorem 1.3 is an essential ingredient of the proof. In view of the universality of $S$ as attractor to all defocused solutions, and in continuation of Theorem 1.3, it is an important open problem to understand the behavior of $S(t)$ as $t \rightarrow+\infty$. For the mass critical (NLS), the explicit formula (1.6) ensures that $S_{\mathrm{NLS}}$ scatters as $t \rightarrow \infty$, and hence it is a connection from $+\infty$ to 0 . For $(\mathrm{gKdV})$, the decay in space (1.9) of $S(t, x)$ on the left, combined with Theorem 1.2, ensures that $S(t)$ is globally defined for $t>0$, but scattering as $t \rightarrow+\infty$ is an open problem 3 . We conjecture that $S(t)$ actually scatters, and because scattering is an open in $L^{2}$ property, [16], we obtain the corollary:

Corollary 1.5. Assume that $S(t)$ scatters as $t \rightarrow+\infty$. Then any solution in the (Exit) scenario is global for positive time and scatters as $t \rightarrow+\infty$.

Related rigidity theorems near the solitary wave were recently obtained by Nakanishi and Schlag [47, [48] for super critical wave and Schrödinger equations using the invariant set methods of Berestycki, Cazenave [2], the Kenig-Merle concentration compactness approach [14, the classification of minimal dynamics [10], [11, [12] and a further "no return" lemma in the (Exit) regime. This approach relies on the virial algebra which is not known for $(\mathrm{gKdV})$.

We expect the strategy of the proof of Theorem 1.4 reducing the dynamics of defocused solutions to the sole description of the minimal mass solution, to be quite general.

\footnotetext{
${ }^{2}$ remember that it blows up backwards in time.

${ }^{3}$ by scattering for $(\mathrm{gKdV})$, we mean that there exists a solution $v(t, x)$ to the Airy equation $\partial_{t} v+v_{x x x}=0$ such that $\lim _{t \rightarrow+\infty}\|S(t)-v(t)\|_{L^{2}}=0$.
} 
Aknowledgements: The authors would like to thank the anonymous referees for their very useful corrections and comments.

P.R is supported by the French ERC/ANR project SWAP. This work is also supported by the project ERC 291214 BLOWDISOL. This work was completed when P.R was visiting the MIT Mathematics Department, Boston, which he would like to thank for its kind hospitality.

Notation: We introduce the generator of $L^{2}$ scaling:

$$
\Lambda f=\frac{1}{2} f+y f^{\prime} .
$$

We note the $L^{2}$ scalar product:

$$
(f, g)=\int_{\mathbb{R}} f(x) g(x) d x .
$$

Let the linearized operator close to $Q$ be:

$$
L f=-f^{\prime \prime}+f-5 Q^{4} f .
$$

For a given generic small constant $0<\alpha^{*} \ll 1, \delta\left(\alpha^{*}\right)$ denotes a generic positive small constant with

$$
\delta\left(\alpha^{*}\right) \rightarrow 0 \text { as } \alpha^{*} \rightarrow 0 .
$$

Given $I$ an interval of $\mathbb{R}$, we let $\mathbf{1}_{I}$ denote the characteristic function of $I$.

1.5. Strategy of the proof. Let us give a brief insight into the strategy of the proof of Theorem 1.3 and Theorem 1.4 .

step 1 Modified blow up profiles. We construct the minimal element using a variation of the compactness argument used for the construction of non dispersive objects in [36], [25], [6], [53]. This solution will admit near blow up time a decomposition

$$
u(t, x)=\frac{1}{\lambda^{\frac{1}{2}}(t)}\left(Q_{b(t)}+\varepsilon\right)(s, y) \text { with } \frac{d s}{d t}=\frac{1}{\lambda^{3}}, \quad y=\frac{x-x(t)}{\lambda(t)},
$$

and

$$
\varepsilon(t) \rightarrow 0 \text { in } H^{1} \text { as } t \downarrow 0 .
$$

Here $Q_{b}$ is the slow modulated deformation of the ground state constructed in [33] which formally leads to the dynamical system

$$
\left\{\begin{array} { l } 
{ b _ { s } + 2 b ^ { 2 } = 0 } \\
{ - \frac { \lambda _ { s } } { \lambda } = b }
\end{array} \text { i.e. } \left\{\begin{array}{c}
\lambda(t)=\ell^{*} t \\
b(t)=-\ell^{*} \lambda^{2}(t)
\end{array}\right.\right.
$$

and hence the blow up speed (1.8).

step 2 The formal argument. Following [36, [53, we could build the minimal element by considering the solution $u_{n}(t)$ to $(\mathrm{gKdV})$ with data

$$
u\left(t_{n}\right)=\frac{1}{\lambda\left(t_{n}\right)^{\frac{1}{2}}} Q_{b\left(t_{n}\right)}\left(\frac{x-x\left(t_{n}\right)}{\lambda\left(t_{n}\right)}\right) \text { with } \lambda\left(t_{n}\right)=\ell^{*} t_{n}, \quad b\left(t_{n}\right)=-\ell^{*} \lambda^{2}\left(t_{n}\right)
$$

and show that there exists a time $t_{0}>0$ independent of $n$ such that

$$
\left\|u_{n}\left(t_{0}\right)\right\|_{H^{1}} \lesssim 1 \text { as } t_{n} \downarrow 0 \text {. }
$$

Such an estimate is the heart of the proof and would be a consequence of the fine monotonicity properties exhibited in [33]. Passing to the limit $t_{n} \rightarrow 0$ automatically produces the expected blow up element. 
We will argue slightly differently and propose a scheme adapted to the proof of both Theorem 1.3 and Theorem 1.4 and which as in [45] illustrates the fact the minimal element can be obtained as limiting sequences of defocusing solutions. Indeed, we pick a sequence of well prepared initial data

$$
u_{n}(0)=Q_{b_{n}(0)}, \quad b_{n}(0)=-\frac{1}{n}
$$

which by construction have sub critical mass

$$
\left\|u_{n}(0)\right\|_{L^{2}}=\|Q\|_{L^{2}}-\frac{c}{n}+o\left(\frac{1}{n}\right) .
$$

Such solutions are automatically in the (Exit) regime of Theorem 1.1. Moreover, we have from [33] a complete description of the flow for $t \in\left[0, t_{n}^{*}\right]$ i.e. the solution admits a decomposition

$$
u_{n}(t, x)=\frac{1}{\lambda_{n}^{\frac{1}{2}}(t)}\left(Q_{b_{n}(t)}+\varepsilon_{n}\right)\left(t, \frac{x-x_{n}(t)}{\lambda_{n}(t)}\right)
$$

where to leading order the modulation equations for $\left(b_{n}, \lambda_{n}\right)$ are given by

$$
\frac{b_{n}(t)}{\lambda_{n}^{2}(t)} \sim b_{n}(0)=-\frac{1}{n}, \quad\left(\lambda_{n}\right)_{t} \sim-b_{n}(0)
$$

i.e.

$$
\lambda_{n}(t) \sim 1-b_{n}(0) t, \quad b_{n}(t) \sim b_{n}(0) \lambda_{n}^{2}(t) .
$$

The (Exit) time $t_{n}^{*}$ is the one for which the solution moves strictly away from the solitary wave which in our setting is equivalent to

$$
b_{n}\left(t_{n}^{*}\right)=-\alpha^{*}
$$

independent of $n$. This in particular allows us to compute $t_{n}^{*}$ and show using (1.12) that the solution defocuses:

$$
\lambda_{n}^{2}\left(t_{n}^{*}\right) \sim \frac{b_{n}\left(t_{n}^{*}\right)}{b_{n}(0)} \sim n \alpha^{*} \text { as } n \rightarrow+\infty .
$$

We therefore renormalize the flow at $t_{n}^{*}$ and consider the solution to $(\mathrm{gKdV})$ with data at $t_{n}^{*}$ given by the renormalized $u_{n}$ at $t_{n}^{*}$, explicitly:

$$
v_{n}(\tau, x)=\lambda_{n}^{\frac{1}{2}}\left(t_{n}^{*}\right) u_{n}\left(t_{\tau}, \lambda_{n}\left(t_{n}^{*}\right) x+x_{n}\left(t_{n}^{*}\right)\right), \quad t_{\tau}=t_{n}^{*}+\tau \lambda_{n}^{3}\left(t_{n}^{*}\right) .
$$

Then $v_{n}$ admits from direct check a decomposition

$$
v_{n}(\tau, x)=\frac{1}{\lambda_{v_{n}}(\tau)^{\frac{1}{2}}}\left(Q_{b_{v_{n}}}+\varepsilon_{v_{n}}\right)\left(\tau, \frac{x-x_{v_{n}(\tau)}}{\lambda_{v_{n}(\tau)}}\right)
$$

with from the symmetries of the flow

$$
\lambda_{v_{n}}(\tau)=\frac{\lambda_{n}\left(t_{\tau}\right)}{\lambda_{n}\left(t_{n}^{*}\right)}, \quad x_{v_{n}}(\tau)=\frac{x_{n}\left(t_{\tau}\right)-x_{n}\left(t_{n}\right)}{\lambda_{n}\left(t_{n}^{*}\right)}, \quad b_{v_{n}}(\tau)=b_{n}\left(t_{\tau}\right), \quad \varepsilon_{v_{n}}(\tau)=\varepsilon_{n}\left(t_{\tau}\right) .
$$

The renormalized parameters can be computed approximatively using (1.12):

$$
\begin{aligned}
\lambda_{v_{n}}(\tau) & \sim \frac{\lambda_{n}\left(t_{n}^{*}+\tau \lambda_{n}^{3}\left(t_{n}^{*}\right)\right)}{\lambda_{n}\left(t_{n}^{*}\right)} \sim \frac{1}{\lambda_{n}\left(t_{n}^{*}\right)}\left[1-b_{n}(0)\left(t_{n}^{*}+\tau \lambda_{n}^{3}\left(t_{n}^{*}\right)\right)\right] \\
& \sim \frac{1}{\lambda_{n}\left(t_{n}^{*}\right)}\left[\lambda_{n}\left(t_{n}^{*}\right)-\tau b_{n}(0) \lambda_{n}^{3}\left(t_{n}^{*}\right)\right]=1-\tau b_{n}(0) \lambda_{n}^{2}\left(t_{n}^{*}\right) \\
& =1-\tau b_{n}\left(t_{n}^{*}\right)=1+\tau \alpha^{*} .
\end{aligned}
$$

Letting $n \rightarrow+\infty$, we therefore expect to extract a weak limit $v_{n}(0) \rightarrow v(0)$ such that the corresponding solution $v(\tau)$ to $(\mathrm{gKdV})$ has minimal mass $\|v(0)\|_{L^{2}}=\|Q\|_{L^{2}}$ 
and blows up backwards at some finite time $\tau^{*} \sim-\frac{1}{\alpha^{*}}$ with the blow up speed $\lambda_{v}(\tau) \sim \tau-\tau^{*}$ i.e (1.8).

The extraction of the weak limit now requires sharp controls on the remaining radiation $\varepsilon_{v_{n}}$. Here an essential use is made of the fact that the set of data $u_{n}(0)$ is well prepared as this induces uniform bounds for $\varepsilon_{v_{n}}(0)=\varepsilon_{u_{n}}\left(t_{n}^{*}\right)$ in $H^{1}$ and allow us to use the $H^{1}$ weak continuity of the flow in the limiting process.

step 3 Solutions in the (Exit) regime. The proof of Theorem 1.4 follows similarly considering sequences of data $\left(u_{0}\right)_{n}$ with $\left\|\left(u_{0}\right)_{n}\right\|_{L^{2}} \rightarrow\|Q\|_{L^{2}}$ such that the corresponding solution to $(\mathrm{gKdV})$ is in the (Exit) regime. We write explicitly the solution at the (Exit) time in the form (1.11), renormalize the flow and now aim at extracting a weak limit as $n \rightarrow+\infty$. The architecture of the proof is similar, except that we have lost the fact that the data is well prepared which destroys the uniform $H^{1}$ bound on $v_{n}(0)$. We therefore use two new tools: a concentration compactness argument on sequences of solutions in the critical $L^{2}$ space in the spirit of [14 using the tools developed in [18, which allows us to extract a non trivial weak limit with suitable dynamical controls; refined local $H^{1}$ bounds on $v_{n}(\tau)$ in order to ensure that the $L^{2}$ limit is in fact also in $H^{1}$. Hence the weak limit is a minimal mass $H^{1}$ blow up element.

step 4 Uniqueness. It remains to prove the uniqueness in $H^{1}$ of the minimal element. This is a delicate problem and here we adapt the direct dynamical approach developed in [53]. The first step is to show that any $H^{1}$ minimal blow up element blows up with the blow up speed (1.8). Here the proof relies first on exponential decay estimates of minimal elements proved in [28] which allow us in a second time to enter the monotonicity machinery developed in [33. Once the blow up speed is known, one may integrate the flow backwards from the singularity and show that the blow up element is close in a strong sense to the $S(t)$ minimal element previously constructed. It remains to show that the difference is exactly zero. This requires revisiting the monotonicity properties for the difference of two such solutions, and showing that the previously obtained a priori bounds on the solution implies that the difference is exactly zerd4. Let us insist that as in [53], [45], we are forced to work with a finite order approximation of the solution 5 and therefore this step is always delicate.

\section{Nonlinear profiles and decomposition close to the soliton}

We collect in this section a number of tools which can be explicitly found in the literature and which we will use in the proof of the main results. We start with recalling the status of scattering theory and profiles decomposition in the critical $L^{2}$ space for $(\mathrm{gKdV})$. We then recall the nonlinear decomposition of the flow for data near the ground state, and the main monotonicity formula at the heart of the analysis in [33] and which will play again a distinguished role in the analysis.

2.1. Cauchy problem and scattering from [15]. We use in the paper the terminology strong solution in the sense of Kenig, Ponce, Vega [15]. For $u_{0} \in L^{2}$, we denote by $v(t)=W(t) u_{0}$ the solution of the Airy equation $v_{t}+v_{x x x}=0$ with

\footnotetext{
${ }^{4}$ This equivalently means that the integration of the flow from blow up time defining the minimal blow up element is a contraction mapping in a suitable function space.

5 and not arbitrarily degenerate as in 3 for example.
} 
$v(0)=v_{0}$. The following space-time Strichartz type estimate proved in [15] is essential in the resolution of the Cauchy problem for (1.1) in $L^{2}$ and $H^{1}$ :

$$
\left\|W(t) v_{0}\right\|_{L_{x}^{5} L_{t}^{10}} \lesssim\left\|v_{0}\right\|_{L^{2}}
$$

We recall the following classical results.

Theorem 2.1 (Kenig, Ponce, Vega [15]).

(i) $L^{2}$ theory. The Cauchy problem (1.1) is locally well-posed in $L^{2}$ : for all $u_{0} \in L^{2}$, there exists a unique $L^{2}$ solution of (1.1) defined on a maximal interval of existence $[0, T)$. There is continuous dependance on the data in $L^{2}$, and there holds the blow up alternative:

$$
T<+\infty \text { implies }\|u\|_{L_{x}^{5} L_{T}^{10}}=+\infty .
$$

Moroever, there exists $\delta>0$ such that $\left\|u_{0}\right\|_{L^{2}}<\delta$ implies that the solution is global with $\|u\|_{L_{x}^{5} L_{\infty}^{10}}<+\infty$.

(ii) $H^{1}$ theory. The Cauchy problem (1.1) is locally well-posed in $H^{1}$ : for all $u_{0} \in$ $H^{1}$, there exists a unique $H^{1}$ solution of (1.1) defined on a maximal interval of existence $[0, T)$. There is continuous dependance on the data in $H^{1}$, and there holds the blow up alternative:

$$
T<+\infty \text { implies } \lim _{t \uparrow T}\left\|\partial_{x} u\right\|_{L^{2}}=+\infty .
$$

(iii) Scattering and stability of scattering. Let $u(t)$ be a global $L^{2}$ solution of (1.1). If $\|u\|_{L_{x}^{5} L_{\infty}^{10}}<+\infty$, then the solution $u(t)$ scatters at $+\infty$ i.e. there exists $v_{0}^{+} \in L^{2}$ such that

$$
\lim _{t \rightarrow \infty}\left\|u(t)-W(t) v_{0}^{+}\right\|_{L^{2}}=0
$$

The set $\mathcal{S}=\left\{u_{0} \in L^{2}\right.$ such that $u(t)$ is global and scatters at $\left.+\infty\right\}$ is open in $L^{2}$.

Point (iii) of Theorem 2.1 follows from [15] and standard arguments (see e.g. [14] for similar arguments in the case of nonlinear Schrödinger equation), and means that scattering is a stable regime without any assumption of size on the solution.

We now recall the known results from [54] on profile decomposition in the critical space of sequences of solutions to the Airy equation which describes the lack of compactness of the Strichartz estimate (2.1). For any $x_{0} \in \mathbb{R}$ and $\lambda>0$, define the operator $g_{x_{0}, \lambda}: L^{2}(\mathbb{R}) \rightarrow L^{2}(\mathbb{R})$

$$
\left[g_{x_{0}, \lambda} f\right](x):=\lambda^{-\frac{1}{2}} f\left(\lambda^{-1}\left(x-x_{0}\right)\right) .
$$

Definition 2.2. For $j \neq k, \Gamma_{n}^{j}=\left(\lambda_{n}^{j}, \xi_{n}^{j}, x_{n}^{j}, t_{n}^{j}\right)_{n \geq 1}$ and $\Gamma_{n}^{k}=\left(\lambda_{n}^{k}, \xi_{n}^{k}, x_{n}^{k}, t_{n}^{k}\right)_{n \geq 1}$ in $(0, \infty) \times \mathbb{R}^{3}$ are orthogonal if one of the following holds

$$
\begin{aligned}
& \text { - } \lim _{n \rightarrow \infty}\left(\frac{\lambda_{n}^{j}}{\lambda_{n}^{k}}+\frac{\lambda_{n}^{k}}{\lambda_{n}^{j}}+\lambda_{n}^{j}\left|\xi_{n}^{j}-\xi_{n}^{k}\right|\right)=\infty \\
& \text { - }\left(\lambda_{n}^{j}, \xi_{n}^{j}\right)=\left(\lambda_{n}^{k}, \xi_{n}^{k}\right) \text { and } \\
& \lim _{n \rightarrow \infty}\left(\frac{\left|t_{n}^{k}-t_{n}^{j}\right|}{\left(\lambda_{n}^{j}\right)^{3}}+\frac{3\left|\left(t_{n}^{k}-t_{n}^{j}\right) \xi_{n}^{j}\right|}{\left(\lambda_{n}^{j}\right)^{2}}+\frac{\left|x_{n}^{j}-x_{n}^{k}+3\left(t_{n}^{j}-t_{n}^{k}\right)\left(\xi_{n}^{j}\right)^{2}\right|}{\lambda_{n}^{j}}\right)=\infty .
\end{aligned}
$$

Lemma 2.3 (Profile decomposition [54]). Let $\left\{u_{n}\right\}_{n \geq 1}$ be a sequence of real-valued functions bounded in $L^{2}$. Then, after passing to a subsequence if necessary, there exist (complex) $L^{2}$ functions $\left\{\phi^{j}\right\}_{j \geq 1}$, and a family of orthogonal sequences $\Gamma_{n}^{j}=$ $\left(\lambda_{n}^{j}, \xi_{n}^{j}, x_{n}^{j}, t_{n}^{j}\right) \in(0, \infty) \times \mathbb{R}^{3}$ such that for all $J \geq 1$,

$$
u_{n}=\sum_{1 \leq j \leq J} e^{-t_{n}^{j} \partial_{x}^{3}}\left(g_{x_{n}^{j}, \lambda_{n}^{j}}\left[\operatorname{Re}\left(e^{i x \xi_{n}^{j} \lambda_{n}^{j}} \phi^{j}\right)\right]\right)+w_{n}^{J},
$$


where $\xi_{n}^{j}$ satisfy the following property: for any $1 \leq j \leq J$, either $\xi_{n}^{j}=0$ for all $n \geq 1$, or $\xi_{n}^{j} \lambda_{n}^{j} \rightarrow \infty$ as $n \rightarrow \infty$. Here, $w_{n}^{J} \in L^{2}(\mathbb{R})$ is real-valued and

$$
\lim _{J \rightarrow \infty} \limsup _{n \rightarrow \infty}\left\{\left\|\left|\partial_{x}\right|^{1 / 6} e^{-t \partial_{x}^{3}} w_{n}^{J}\right\|_{L_{t, x}^{6}(\mathbb{R} \times \mathbb{R})}+\left\|e^{-t \partial_{x}^{3}} w_{n}^{J}\right\|_{L_{x}^{5} L_{t}^{10}(\mathbb{R} \times \mathbb{R})}\right\}=0 .
$$

Moreover, for any $J \geq 1$,

$$
\lim _{n \rightarrow \infty}\left\{\left\|u_{n}\right\|_{L^{2}}^{2}-\sum_{1 \leq j \leq J}\left\|\operatorname{Re}\left[e^{i x \xi_{n}^{j} \lambda_{n}^{j}} \phi^{j}\right]\right\|_{L^{2}}^{2}-\left\|w_{n}^{J}\right\|_{L^{2}}^{2}\right\}=0 .
$$

Using this lemma for the study of the nonlinear flow (1.1) requires a suitable perturbation theory:

Lemma 2.4 ( $L^{2}$ perturbation theory [18]). Let $I$ be an interval of $\mathbb{R}, 0 \in I$, and let $\tilde{u}$ be an $L^{2}$ solution of

$$
u_{t}+\left(u_{x x}+u^{5}\right)_{x}=e_{x}
$$

on $I \times \mathbb{R}$ for some function e. Assume that

$$
\|\tilde{u}\|_{L_{t}^{\infty} L_{x}^{2}(I \times \mathbb{R})}+\|\tilde{u}\|_{L_{x}^{5} L_{t}^{10}(I \times \mathbb{R})} \leq M
$$

for some $M>0$. Let $u(0) \in L^{2}$ be such that

$$
\begin{gathered}
\|u(0)-\tilde{u}(0)\|_{L^{2}} \leq M^{\prime}, \\
\left\|e^{-t \partial^{3}}(u(0)-\tilde{u}(0))\right\|_{L_{x}^{5} L_{t}^{10}(I \times \mathbb{R})}+\|e\|_{L_{x}^{1} L_{t}^{2}(I \times \mathbb{R})} \leq \epsilon,
\end{gathered}
$$

for $M^{\prime}>0$ and for some small $0<\epsilon<\epsilon_{0}\left(M, M^{\prime}\right)$. Then, the solution $u(t)$ of (1.1) corresponding to $u\left(t_{0}\right)$ is defined on $I$ and there holds the bound:

$$
\|u-\tilde{u}\|_{L_{x}^{5} L_{t}^{10}(I \times \mathbb{R})}+\|u-\tilde{u}\|_{L_{t}^{\infty} L_{x}^{2}(I \times \mathbb{R})} \leq C\left(M, M^{\prime}\right) \epsilon .
$$

2.2. Approximate self similar profiles. We recall the existence of suitable approximate self similar solutions which give the leading order profile of solutions with data near $Q$. The specific sutrcture of these profiles drives both the blow speed in the (Blow up) regime and the speed of defocalization in the (Exit) regime. Let $\mathcal{Y}$ be the set of functions $f \in \mathcal{C}^{\infty}(\mathbb{R}, \mathbb{R})$ such that

$$
\forall k=0,1,2 \ldots, \exists C_{k}, r_{k}>0, \forall y \in \mathbb{R}, \quad\left|f^{(k)}(y)\right| \leq C_{k}(1+|y|)^{r_{k}} e^{-|y|} .
$$

Let $\chi \in \mathcal{C}^{\infty}(\mathbb{R})$ be such that $0 \leq \chi \leq 1, \chi^{\prime} \geq 0$ on $\mathbb{R}, \chi \equiv 1$ on $[-1,+\infty), \chi \equiv 0$ on $(-\infty,-2]$. Define

$$
\chi_{b}(y)=\chi\left(|b|^{\gamma} y\right), \quad \gamma=\frac{3}{4} .
$$

Lemma 2.5 (Approximate self-similar profiles $Q_{b}$, [33]). There exists a unique smooth function $P$ such that $P^{\prime} \in \mathcal{Y}$ and

$$
\begin{gathered}
(L P)^{\prime}=\Lambda Q, \quad \lim _{y \rightarrow-\infty} P(y)=\frac{1}{2} \int Q, \quad \lim _{y \rightarrow+\infty} P(y)=0, \\
(P, Q)=\frac{1}{16}\left(\int Q\right)^{2}>0, \quad\left(P, Q^{\prime}\right)=0 .
\end{gathered}
$$

Moreover, the localized approximate profile:

$$
Q_{b}(y)=Q(y)+b \chi_{b}(y) P(y),
$$


satisfies:

(i) Estimates on $Q_{b}$ : For all $y \in \mathbb{R}$,

$$
\begin{aligned}
& \left|Q_{b}(y)\right| \lesssim e^{-|y|}+|b|\left(\mathbf{1}_{[-2,0]}\left(|b|^{\gamma} y\right)+e^{-\frac{|y|}{2}}\right), \\
& \left|Q_{b}^{(k)}(y)\right| \lesssim e^{-|y|}+|b| e^{-\frac{|y|}{2}}+|b|^{1+k \gamma} \mathbf{1}_{[-2,-1]}\left(|b|^{\gamma} y\right), \quad \text { for } k \geq 1,
\end{aligned}
$$

(ii) Equation of $Q_{b}$ : let

$$
-\Psi_{b}=\left(Q_{b}^{\prime \prime}-Q_{b}+Q_{b}^{5}\right)^{\prime}+b \Lambda Q_{b}
$$

then, for all $y \in \mathbb{R}$,

$$
\begin{aligned}
& \left|\Psi_{b}(y)\right| \lesssim|b|^{1+\gamma} \mathbf{1}_{[-2,-1]}\left(|b|^{\gamma} y\right)+b^{2}\left(e^{-\frac{|y|}{2}}+\mathbf{1}_{[-2,0]}\left(|b|^{\gamma} y\right)\right), \\
& \left|\Psi_{b}^{(k)}(y)\right| \lesssim|b|^{1+(k+1) \gamma} \mathbf{1}_{[-2,-1]}\left(|b|^{\gamma} y\right)+b^{2} e^{-\frac{|y|}{2}}, \quad \text { for } k \geq 1 .
\end{aligned}
$$

(iii) Mass and energy properties of $Q_{b}$ :

$$
\begin{aligned}
& \left|\int Q_{b}^{2}-\left(\int Q^{2}+2 b \int P Q\right)\right| \lesssim|b|^{2-\gamma}, \\
& \left|E\left(Q_{b}\right)+b \int P Q\right| \lesssim b^{2} .
\end{aligned}
$$

2.3. Geometrical decomposition of the flow. Let $u \in \mathcal{C}^{0}\left(\left[0, t_{0}\right], H^{1}\right)$ be a solution of (1.1) close in $L^{2}$ to the manifold of solitary waves i.e., we assume that there exist $\left(\lambda_{1}(t), x_{1}(t)\right) \in \mathbb{R}_{+}^{*} \times \mathbb{R}$ and $\varepsilon_{1}(t)$ such that

$$
\forall t \in\left[0, t_{0}\right], \quad u(t, x)=\frac{1}{\lambda_{1}^{\frac{1}{2}}(t)}\left(Q+\varepsilon_{1}\right)\left(t, \frac{x-x_{1}(t)}{\lambda_{1}(t)}\right)
$$

with

$$
\forall t \in\left[0, t_{0}\right], \quad\left\|\varepsilon_{1}(t)\right\|_{L^{2}}+\left(\int\left(\partial_{y} \varepsilon_{1}\right)^{2} e^{-\frac{|y|}{2}} d y\right)^{\frac{1}{2}} \leq \kappa_{0}
$$

for some small enough universal constant $\kappa_{0}>0$. This decomposition is refined using the $Q_{b}$ profiles and a standard modulation argument.

Lemma 2.6 (Decomposition and $H^{1}$ properties, 33]). Assume (2.19).

(i) Decomposition: There exist $\mathcal{C}^{1}$ functions $(\lambda, x, b):\left[0, t_{0}\right] \rightarrow(0,+\infty) \times \mathbb{R}^{2}$ such that

$$
\forall t \in\left[0, t_{0}\right], \quad \varepsilon(t, y)=\lambda^{\frac{1}{2}}(t) u(t, \lambda(t) y+x(t))-Q_{b(t)}(y)
$$

satisfies the orthogonality conditions

$$
(\varepsilon(t), y \Lambda Q)=(\varepsilon(t), \Lambda Q)=(\varepsilon(t), Q)=0 .
$$

and

$$
\|\varepsilon(t)\|_{L^{2}}+|b(t)| \lesssim \delta\left(\kappa_{0}\right), \quad\|\varepsilon(t)\|_{H^{1}} \lesssim \delta\left(\left\|\varepsilon_{1}(t)\right\|_{H^{1}}\right) .
$$

(ii) Equation of $\varepsilon$ : Let

$$
s=\int_{0}^{t} \frac{d t^{\prime}}{\lambda^{3}\left(t^{\prime}\right)} \quad \text { and } \quad s_{0}=s\left(t_{0}\right)
$$

For all $s \in\left[0, s_{0}\right]$,

$$
\begin{aligned}
\varepsilon_{s}-(L \varepsilon)_{y}+b \Lambda \varepsilon & =\left(\frac{\lambda_{s}}{\lambda}+b\right)\left(\Lambda Q_{b}+\Lambda \varepsilon\right)+\left(\frac{x_{s}}{\lambda}-1\right)\left(Q_{b}+\varepsilon\right)_{y} \\
& +\Phi_{b}+\Psi_{b}-\left(R_{b}(\varepsilon)\right)_{y}-\left(R_{\mathrm{NL}}(\varepsilon)\right)_{y},
\end{aligned}
$$


where $\Psi_{b}$ is defined in (2.13) and

$$
\begin{aligned}
& \Phi_{b}=-b_{s}\left(\chi_{b}+\gamma y\left(\chi_{b}\right)_{y}\right) P, \\
& R_{b}(\varepsilon)=5\left(Q_{b}^{4}-Q^{4}\right) \varepsilon, \quad R_{\mathrm{NL}}(\varepsilon)=\left(\varepsilon+Q_{b}\right)^{5}-5 Q_{b}^{4} \varepsilon-Q_{b}^{5} .
\end{aligned}
$$

(iii) Estimates induced by the conservation laws: on $\left[0, s_{0}\right]$, there holds

$$
\begin{gathered}
\|\varepsilon(s)\|_{L^{2}}^{2} \lesssim|b(s)|+\left|\int u_{0}^{2}-\int Q^{2}\right| \\
\left|2 \lambda^{2}(s) E_{0}+\frac{b(s)}{8}\|Q\|_{L^{1}}^{2}-\left\|\varepsilon_{y}(s)\right\|_{L^{2}}^{2}\right| \lesssim b^{2}(s)+\|\varepsilon(s)\|_{L^{2}}^{2}+\delta\left(\|\varepsilon\|_{L^{2}}\right)\left\|\varepsilon_{y}(s)\right\|_{L^{2}}^{2} .
\end{gathered}
$$

(iv) Rough modulation equations: on $\left[0, s_{0}\right]$,

$$
\begin{aligned}
& \left|\frac{\lambda_{s}}{\lambda}+b\right|+\left|\frac{x_{s}}{\lambda}-1\right| \lesssim\left(\int \varepsilon^{2} e^{-\frac{|y|}{10}}\right)^{\frac{1}{2}}+b^{2} \\
& \left|b_{s}+2 b^{2}\right| \lesssim|b|\left(\int \varepsilon^{2} e^{-\frac{|y|}{10}}\right)^{\frac{1}{2}}+|b|^{3}+\int \varepsilon^{2} e^{-\frac{|y|}{10}}
\end{aligned}
$$

(v) Minimal mass: if in addition, $\|u(t)\|_{L^{2}}=\|Q\|_{L^{2}}$ then $E_{0}=E\left(u_{0}\right) \geq 0$ and on $\left[0, s_{0}\right]$,

$$
b(s) \leq 0, \quad E_{0} \lambda^{2}(s) \lesssim|b(s)|+\|\varepsilon(s)\|_{H^{1}}^{2} \lesssim E_{0} \lambda^{2}(s) .
$$

The proof of Lemma 2.6] is given in [33], except (2.31) which we prove now.

Proof of (2.31). Using the decomposition (2.20), one has

$$
\int u^{2}=\int Q_{b}^{2}+\int \varepsilon^{2}+2 \int \varepsilon Q_{b}
$$

Since $(\varepsilon, Q)=0$, and $\chi_{b}(y)=\chi\left(|b|^{\frac{3}{4}} y\right)$,

$$
\left|\left(\varepsilon, Q_{b}\right)\right|=|b|\left|\left(\varepsilon, P \chi_{b}\right)\right| \lesssim|b|^{\frac{5}{8}}\left(\int \varepsilon^{2}\right)^{\frac{1}{2}} \lesssim|b|^{\frac{1}{8}} \int \varepsilon^{2}+|b|^{\frac{9}{8}} .
$$

Moreover, by (2.16),

$$
\int Q_{b}^{2}=\int Q^{2}+2 \int P Q+O\left(|b|^{\frac{5}{4}}\right) .
$$

Thus, we obtain in general

$$
\int u^{2}=\int Q^{2}+2 b \int P Q+\int \varepsilon^{2}+O\left(|b|^{\frac{1}{8}}\right)\left(|b|+\int \varepsilon^{2}\right) .
$$

In particular, using the minimal mass assumption $\int u^{2}=\int Q^{2}$,

$$
2 b(P, Q)+\int \varepsilon^{2}=\delta\left(\kappa_{0}\right)\left(|b|+\int \varepsilon^{2}\right),
$$


which implies $b \leq 0$. Now we write the conservation of energy using $(\varepsilon, Q)=0$ and (2.17):

$$
\begin{aligned}
2 \lambda^{2} E_{0} & =2 E\left(Q_{b}\right)-2 \int \varepsilon\left(Q_{b}\right)_{y y}+\int \varepsilon_{y}^{2}-\frac{1}{3} \int\left(\left(Q_{b}+\varepsilon\right)^{6}-Q_{b}^{6}\right) \\
& =-2 b \int P Q+O\left(b^{2}\right)+\int \varepsilon_{y}^{2}-5 \int Q^{4} \varepsilon^{2}-\frac{1}{3} \int \varepsilon^{6} \\
& -2 \int \varepsilon\left[\left(Q_{b}-Q\right)_{y y}+\left(Q_{b}^{5}-Q^{5}\right)\right]+5 \int\left(Q^{4}-Q_{b}^{4}\right) \varepsilon^{2} \\
& -\frac{1}{3} \int\left[\left(Q_{b}+\varepsilon\right)^{6}-Q_{b}^{6}-6 Q_{b}^{5} \varepsilon-15 Q_{b}^{4} \varepsilon^{2}-\varepsilon^{6}\right] .
\end{aligned}
$$

We estimate the nonlinear terms using the Sobolev bound

$$
\|\varepsilon\|_{L^{\infty}} \lesssim\left\|\varepsilon_{y}\right\|_{L^{2}}^{\frac{1}{2}}\|\varepsilon\|_{L^{2}}^{\frac{1}{2}},
$$

and thus

$$
2 \lambda^{2} E_{0}=-2 b \int P Q+\int \varepsilon_{y}^{2}-5 \int Q^{4} \varepsilon^{2}+O\left(|b|^{\frac{1}{8}}+\|\varepsilon\|_{H^{1}}\right)\left(|b|+\int \varepsilon^{2}\right) .
$$

Combining with (2.34), we obtain:

$$
2 \lambda^{2} E_{0}=(L \varepsilon, \varepsilon)+\delta\left(\kappa_{0}\right)\left(|b|+\|\varepsilon\|_{H^{1}}^{2}\right) .
$$

The choice of orthogonality conditions on $\varepsilon$ ensures (see Lemma 2.1 in [33]) the coercivity of the linearized energy, i.e. $(L \varepsilon, \varepsilon) \gtrsim\|\varepsilon\|_{H^{1}}^{2}$ and thus

$$
\|\varepsilon\|_{H^{1}}^{2} \lesssim \lambda^{2} E_{0}+\delta\left(\kappa_{0}\right)|b|,
$$

which combined with (2.34) implies (2.31).

The modulation equations can be sharpened under an additional $L^{1}$ control of the solution.

Lemma 2.7 (Refined laws for $H^{1}$ solution with decay, [33]). Under the assumptions of Lemma 2.6, assume moreover the uniform $L^{1}$ control on the right:

$$
\forall t \in\left[0, t_{0}\right], \quad \int_{y>0}|\varepsilon(t)| \lesssim \delta\left(\kappa_{0}\right),
$$

then the quantities $J_{1}$ and $J_{2}$ below are well-defined and satisfy on $\left[0, t_{0}\right]$ :

- Law of $\lambda$ : let

$$
\rho_{1}(y)=\frac{4}{\left(\int Q\right)^{2}} \int_{-\infty}^{y} \Lambda Q, \quad J_{1}(s)=\left(\varepsilon(s), \rho_{1}\right),
$$

then for some universal constant $c_{1}$,

$$
\left|\frac{\lambda_{s}}{\lambda}+b+c_{1} b^{2}-2\left(\left(J_{1}\right)_{s}+\frac{1}{2} \frac{\lambda_{s}}{\lambda} J_{1}\right)\right| \lesssim \int \varepsilon^{2} e^{-\frac{|y|}{10}}+|b|\left(\int \varepsilon^{2} e^{-\frac{|y|}{10}}\right)^{\frac{1}{2}} .
$$

- Law of b: let

$$
\rho_{2}=\frac{16}{\left(\int Q\right)^{2}}\left(\frac{(\Lambda P, Q)}{\|\Lambda Q\|_{L^{2}}^{2}} \Lambda Q+P-\frac{1}{2} \int Q\right)-8 \rho_{1}, \quad J_{2}(s)=\left(\varepsilon(s), \rho_{2}\right),
$$

then for some universal constant $c_{2}$.

$$
\left|b_{s}+2 b^{2}+c_{2} b^{3}+b\left(\left(J_{2}\right)_{s}+\frac{1}{2} \frac{\lambda_{s}}{\lambda} J_{2}\right)\right| \lesssim \int \varepsilon^{2} e^{-\frac{|y|}{10}}+|b|^{4} .
$$


- Law of $\frac{b}{\lambda^{2}}$ : let

$$
\rho=4 \rho_{1}+\rho_{2}, \quad J=(\varepsilon, \rho)
$$

then $\rho \in \mathcal{Y}$ so that $|J| \lesssim\left(\int \varepsilon^{2} e^{-\frac{|y|}{10}}\right)^{\frac{1}{2}}$ and for $c_{0}=c_{2}-2 c_{1}$,

$$
\left|\frac{d}{d s}\left(\frac{b}{\lambda^{2}}\right)+\frac{b}{\lambda^{2}}\left(J_{s}+\frac{1}{2} \frac{\lambda_{s}}{\lambda} J\right)+c_{0} \frac{b^{3}}{\lambda^{2}}\right| \lesssim \frac{1}{\lambda^{2}}\left(\int \varepsilon^{2} e^{-\frac{|y|}{10}}+|b|^{4}\right) .
$$

2.4. Weak $H^{1}$ stability of the decomposition. The geometrical decomposition of Lemma 2.6 is stable by weak $H^{1}$ limits.

Lemma $2.8\left(H^{1}\right.$-weak stability and convergence of the parameters [26]). Let $u_{n}(0)$ be a sequence of $H^{1}$ initial data such that

$$
u_{n}(0) \rightarrow u(0) \in H^{1} \quad \text { as } n \rightarrow+\infty .
$$

Assume that for some $T_{1}>0$, for all $n$, the corresponding solution $u_{n}$ of (1.1) exists and satisfies (2.19) on $\left[0, T_{1}\right]$. Assume further that the decomposition $\left(\lambda_{n}, x_{n}, b_{n}\right)$ of $u_{n}$ given by Lemma 2.6 satisfies

$$
\forall t \in\left[0, T_{1}\right], \quad 0<c \leq \lambda_{n}(t)<C, \quad \lambda_{n}(0)=1, \quad x_{n}(0)=0 .
$$

Then, the $H^{1}$ solution $u(t)$ of (1.1) corresponding to $u(0)$ exists on $\left[0, T_{1}\right]$, satisfies (2.19) and its decomposition satisfies

$$
\forall t \in\left[0, T_{1}\right], \quad \varepsilon_{n}(t) \rightarrow \varepsilon(t), \quad \lambda_{n}(t) \rightarrow \lambda(t), \quad x_{n}(t) \rightarrow x(t), \quad b_{n}(t) \rightarrow b(t) .
$$

This lemma is similar to a result proved in Lemma 17 and Appendix D of [26], and therefore we omit its proof.

2.5. Main monotonicity functionals from [33]. We now recall the monotonicity formula at the heart of the analysis in [33] and on which we shall heavily rely again. We refer to [33] for a further introduction to the natures of these functionals and the associated ridigity of the flow implied by (2.57).

Let $\varphi, \psi \in C^{\infty}(\mathbb{R})$ be such that:

$$
\begin{gathered}
\varphi(y)=\left\{\begin{array}{l}
e^{y} \text { for } y<-1, \\
1+y \text { for }-\frac{1}{2}<y<\frac{1}{2} \quad, \quad \varphi^{\prime}(y)>0, \quad \forall y \in \mathbb{R}, \\
y^{2} \text { for for } y>2
\end{array}\right. \\
\psi(y)=\left\{\begin{array}{l}
e^{2 y} \text { for } y<-1, \\
1 \text { for } y>-\frac{1}{2}
\end{array}, \quad \psi^{\prime}(y) \geq 0 \quad \forall y \in \mathbb{R} .\right.
\end{gathered}
$$

For $B \geq 100$ to be fixed, let

$$
\psi_{B}(y)=\psi\left(\frac{y}{B}\right), \quad \varphi_{B}=\varphi\left(\frac{y}{B}\right),
$$

and define

$$
\mathcal{N}(s)=\int \varepsilon_{y}^{2}(s, y) \psi_{B}(y) d y+\int \varepsilon^{2}(s, y) \varphi_{B}(y) d y .
$$

Proposition 2.9 (Monotonicity formula, [33]). There exist $\mu>0$ and $0<\kappa^{*}<\kappa_{0}$ such that the following holds for $B>100$ large enough. Assume that $u(t)$ is a solution of (1.1) which satisfies (2.19) on $\left[0, t_{0}\right]$ and thus admits on $\left[0, t_{0}\right]$ a decomposition (2.20) as in Lemma [2.6, Let $s_{0}=s\left(t_{0}\right)$, and assume the following a priori bounds: $\forall s \in\left[0, s_{0}\right]$,

(H1) smallness:

$$
\|\varepsilon(s)\|_{L^{2}}+|b(s)|+\mathcal{N}(s) \leq \kappa^{*}
$$


(H2) comparison between $b$ and $\lambda$ :

$$
\frac{|b(s)|+\mathcal{N}(s)}{\lambda^{2}(s)} \leq \kappa^{*}
$$

(H3) $L^{2}$ weighted bound on the right:

$$
\int_{y>0} y^{10} \varepsilon^{2}(s, x) d x \leq 10\left(1+\frac{1}{\lambda^{10}(s)}\right) .
$$

Let for $j \in\{1,2\}$ :

$$
\mathcal{F}_{j}=\int\left[\varepsilon_{y}^{2} \psi_{B}+\varepsilon^{2}\left(1+\mathcal{J}_{j}\right) \varphi_{B}-\frac{1}{3}\left(\left(\varepsilon+Q_{b}\right)^{6}-Q_{b}^{6}-6 \varepsilon Q_{b}^{5}\right) \psi_{B}\right],
$$

with

$$
\mathcal{J}_{j}=\left(1-J_{1}\right)^{-4 j}-1
$$

Then the following bounds hold on $\left[0, s_{0}\right]$ :

(i) Scaling invariant Lyapounov control:

$$
\frac{d \mathcal{F}_{1}}{d s}+\mu \int\left(\varepsilon_{y}^{2}+\varepsilon^{2}\right) \varphi_{B}^{\prime} \lesssim|b|^{4}
$$

(ii) Scaling weighted $H^{1}$ Lyapounov control:

$$
\frac{d}{d s}\left\{\frac{\mathcal{F}_{2}}{\lambda^{2}}\right\}+\frac{\mu}{\lambda^{2}} \int\left(\varepsilon_{y}^{2}+\varepsilon^{2}\right) \varphi_{B}^{\prime} \lesssim \frac{|b|^{4}}{\lambda^{2}} .
$$

(iii) Pointwise bounds:

$$
\begin{gathered}
\left|J_{1}\right|+\left|J_{2}\right| \lesssim \mathcal{N}^{\frac{1}{2}} \\
\mathcal{N} \lesssim \mathcal{F}_{j} \lesssim \mathcal{N}, \quad j=1,2 .
\end{gathered}
$$

The integration of the modulation equations of Lemma 2.7 with the dispersive bounds of Proposition 2.9 implies the control of the flow by the sole parameter $b$ :

Lemma 2.10 (Control of the flow by $b,[33])$. Under the assumptions of Proposition 2.9, the following hold

(i) Control of the dynamics for $b$. For all $0 \leq s_{1} \leq s_{2}<s_{0}$,

$$
\begin{gathered}
\int_{s_{1}}^{s_{2}} b^{2}(s) d s \lesssim \int\left(\varepsilon_{y}^{2}\left(s_{1}\right) \psi_{B}+\varepsilon^{2}\left(s_{1}\right) \varphi_{B}^{\prime}\right)+\left|b\left(s_{2}\right)\right|+\left|b\left(s_{1}\right)\right|, \\
\left|\frac{b\left(s_{2}\right)}{\lambda^{2}\left(s_{2}\right)}-\frac{b\left(s_{1}\right)}{\lambda^{2}\left(s_{1}\right)}\right| \leq \frac{C^{*}}{10}\left[\frac{b^{2}\left(s_{1}\right)}{\lambda^{2}\left(s_{1}\right)}+\frac{b^{2}\left(s_{2}\right)}{\lambda^{2}\left(s_{2}\right)}+\frac{1}{\lambda^{2}\left(s_{1}\right)} \int\left(\varepsilon_{y}^{2}\left(s_{1}\right) \psi_{B}+\varepsilon^{2}\left(s_{1}\right) \varphi_{B}^{\prime}\right)\right],
\end{gathered}
$$

for some universal constant $C^{*}>0$.

(ii) Control of the scaling dynamics. Let $\lambda_{0}(s)=\lambda(s)\left(1-J_{1}(s)\right)^{2}$. Then on $\left[0, s_{0}\right)$,

$$
\left|\frac{\left(\lambda_{0}\right)_{s}}{\lambda_{0}}+b+c_{1} b^{2}\right| \lesssim \int \varepsilon^{2} e^{-\frac{|y|}{10}}+|b| \mathcal{N}^{\frac{1}{2}}+|b|^{3} .
$$

(iii) Dispersive bounds. For all $0 \leq s_{1} \leq s_{2}<s_{0}$,

$$
\begin{gathered}
\mathcal{N}\left(s_{2}\right)+\int_{s_{1}}^{s_{2}}\left[\int\left(\varepsilon_{y}^{2}+\varepsilon^{2}\right)(s) \varphi_{B}^{\prime}+|b|^{4}(s)\right] d s \lesssim \mathcal{N}\left(s_{1}\right)+\left(\left|b^{3}\left(s_{2}\right)\right|+\left|b^{3}\left(s_{1}\right)\right|\right) .(2.59) \\
\frac{\mathcal{N}\left(s_{2}\right)}{\lambda^{2}\left(s_{2}\right)}+\int_{s_{1}}^{s_{2}}\left[\int\left(\varepsilon_{y}^{2}+\varepsilon^{2}\right)(s) \varphi_{B}^{\prime}+|b|^{4}(s)\right] \frac{d s}{\lambda^{2}(s)} \lesssim \frac{\mathcal{N}\left(s_{1}\right)}{\lambda^{2}\left(s_{1}\right)}+\left[\frac{\left|b^{3}\left(s_{1}\right)\right|}{\lambda^{2}\left(s_{1}\right)}+\frac{\left|b^{3}\left(s_{2}\right)\right|}{\lambda^{2}\left(s_{2}\right)}\right] .
\end{gathered}
$$


2.6. Localization in space and decay properties of minimal mass solutions. Minimal mass blow up solutions have been studied in some details in [30] using tools developed in [28] and [29]. Recall that the main result of [30] is the nonexistence of minimal mass blow up solutions, assuming initial decay in space. In proving this result, several general properties of minimal mass blow up solutions were derived. We gather in the next lemma all useful information which can be deduced from [30] on general minimal mass blow up solutions. Note that at this stage, we do not know whether a minimal mass blow up solution should blow up in finite or infinite time. See Proposition 4.1 in Section 4 for refined information.

Lemma 2.11 (First properties of minimal mass blow up solutions [30]). Let $u(t)$ be a solution of (1.1) defined on $(T, 0]$, which blows up backwards in finite or infinite time $-\infty \leq T<0$. Assume

$$
\int u^{2}(0)=\int Q^{2}
$$

There exists $t_{1}>T$ close to $T$ such that for all $t \in\left(T, t_{1}\right], u(t)$ or $-u(t)$ admits a decomposition $(\lambda(t), x(t), b(t), \varepsilon(t))$ as in Lemma [2.6, with

$$
\lim _{t \rightarrow T} \lambda(s)=0,
$$

and for all $t \in\left(T, t_{1}\right)$,

$$
\int_{T}^{t} \frac{b^{2}\left(t^{\prime}\right)+\int \varepsilon^{2}\left(t^{\prime}, y\right) e^{-\frac{|y|}{10}} d y}{\lambda^{3}\left(t^{\prime}\right)} d t^{\prime}+\mathcal{N}(t)+\|\varepsilon(t)\|_{H^{1}}^{2}+|b(t)| \lesssim \lambda^{2}(t) E_{0} .
$$

Moreover,

$$
\begin{aligned}
& \text { for all } t, t^{\prime} \in\left(T, t_{1}\right), \quad t<t^{\prime} \text { implies } \lambda\left(t^{\prime}\right) \geq \frac{1}{24} \lambda(t), \\
& \text { for all } t \in\left(T, t_{1}\right), \text { for all } y>0, \quad|\varepsilon(t, y)| \lesssim e^{-\frac{y}{2000}}
\end{aligned}
$$

Proof. Let $u(t) \in \mathcal{C}\left((T, 0], H^{1}\right)$ be a general backwards minimal mass blow up solution defined on $(T, 0]$ and blowing up in finite or infinite time $6:-\infty \leq T<0$ :

$$
\lim _{t \rightarrow T}\left\|u_{x}(t)\right\|_{L^{2}}=+\infty .
$$

From standard concentration compactness argument 7 and using the mass and energy conservations, either $u(t)$ or $-u(t)$ satisfies (2.18) and (2.19) for $t$ close to $T$, with in addition

$$
\left\|\varepsilon_{1}(t)\right\|_{H^{1}} \rightarrow 0 \text { as } t \rightarrow T
$$

thanks to the minimal mass assumption. Therefore, possibly considering $-u(t)$ instead of $u(t)$, there exists $t_{0}>T$ such that the solution $u(t)$ admits on $\left(T, t_{0}\right]$ a decomposition given by Lemma 2.6

$$
u(t, x)=\frac{1}{\lambda^{\frac{1}{2}}(t)}\left(Q_{b(t)}+\varepsilon\right)\left(t, \frac{x-x(t)}{\lambda(t)}\right)
$$

with

$$
\forall t \in\left(T, t_{0}\right], \quad|b(t)|+\|\varepsilon(t)\|_{H^{1}} \leq \alpha^{*}
$$

\footnotetext{
${ }^{6}$ Note that the uniqueness statement in Theorem 1.3 (ii) concerns only finite time blow up solutions. Actually, we also prove in this paper the nonexistence of minimal mass solutions blowing up at infinity (see Proposition 4.1 and Section 5). However, we do not treat the case of global minimal mass solutions blowing up only on a subsequence of time.

${ }^{7}$ See for example the lecture notes $[50$.
} 
where $\alpha^{*}>0$ is any small given constant. With this decomposition, the (finite or infinite time) blow up assumption (2.66) is equivalent to: $\lim _{t \rightarrow T} \lambda(s)=0$, and by (2.31),

$$
b(t)<0, \quad E_{0}>0, \quad|b(t)|+\|\varepsilon(t)\|_{H^{1}}^{2} \lesssim \lambda^{2}(t) E_{0} .
$$

Now, we recall results from [30]. First, recall that the solution $u(t, x)$ is decomposed in a different way in 30, Lemma 1 . Indeed, there exist $C^{1}$ functions $\tilde{\lambda}$ and $\tilde{x}$ such that

$$
\tilde{\varepsilon}(t, y)=\tilde{\lambda}^{\frac{1}{2}}(t) u(t, \tilde{\lambda}(t) y+\tilde{x}(t))-Q(y)
$$

satisfies the orthogonality conditions

$$
\int \Lambda Q(y) \tilde{\varepsilon}(t, y) d y=\int y \Lambda Q(y) \tilde{\varepsilon}(t, y) d y=0 .
$$

Note that one easily compares this decomposition with (2.67), in particular, combining the orthogonality conditions of $\varepsilon$ and $\tilde{\varepsilon}$, one obtains

$$
\left|1-\frac{\lambda(t)}{\tilde{\lambda}(t)}\right|+|b(t)| \lesssim\left(\int \tilde{\varepsilon}^{2}(t) e^{-\frac{|y|}{10}} d y\right)^{\frac{1}{2}} .
$$

Under the general assumptions of Lemma 2.11, we now claim that for some $T<$ $t_{1}<t_{0}$,

$$
\text { for all } t^{\prime}, t \in\left(T, t_{1}\right) \text {, if } t \leq t^{\prime} \text { then } \tilde{\lambda}(t) \leq 4 \tilde{\lambda}\left(t^{\prime}\right)
$$

and

$$
\text { for all } t \in\left(T, t_{1}\right) \text {, for all } y>0, \quad|\tilde{\varepsilon}(t, y)| \lesssim e^{-\frac{y}{1000}} .
$$

To prove (2.71) and (2.72), we invoke the arguments of Section 4 in [30]. Recall that the main result of [30], stated in Theorem 1.2 of the present paper, claims forward global existence for minimal mass solutions under the decay assumption (1.7). Unlike Section 3, based on the decay assumption on the initial data, Section 4 of [30] does not make use of this assumption, except when asserting that blow up occurs in finite time. At this point, it is important to note that here time is reversed with respect to [30], thus left and right in space are also reversed (recall that if $u(t, x)$ is solution of (1.1), then $u(-t,-x)$ is also solution of (1.1)). First, using Lemma 4 in [30], one obtains uniform exponential decay on the right in space, on a special sequence of time $t_{n} \rightarrow T$,

$$
\text { for all } y>0, \quad\left|\tilde{\varepsilon}\left(t_{n}, y\right)\right| \lesssim e^{-\frac{y}{1000}} .
$$

Then, combining Step 2 of the proof of Proposition 2 (page 401) of [30], we obtain (2.71), i.e. the almost monotonicity property of $\tilde{\lambda}$ and in return, using Lemma 4 again, the decay property on the right (2.72) for all time.

Note that from (2.69) and (2.72), we have

$$
\int_{y>0} y^{10} \tilde{\varepsilon}^{2}(t, y) d y \rightarrow 0 \quad \text { as } t \rightarrow T .
$$

Now, using further algebra developed page 405 of [30], we claim that, for all $T<t_{2}<t_{1}$,

$$
\int_{t_{2}}^{t_{1}} \frac{\int \tilde{\varepsilon}^{2} e^{-\frac{|y|}{10^{4}}} d y}{\tilde{\lambda}^{3}} d t \lesssim \tilde{\lambda}^{2}\left(t_{1}\right) E_{0}
$$

Indeed, it is proved there that for all $T<t_{2}<t_{1}$,

$$
\int_{t_{2}}^{t_{1}} \frac{\int \tilde{\varepsilon}^{2} e^{-\frac{|y|}{10^{4}}} d y}{\tilde{\lambda}^{3}} d t \lesssim \int \varepsilon^{2}\left(t_{1}\right)+\int \varepsilon^{2}\left(t_{2}\right)+\int_{t_{2}}^{t_{1}} \frac{\left(\int \tilde{\varepsilon} Q\right)^{2}}{\tilde{\lambda}^{3}} d t
$$


and that there exists $\bar{\lambda}(t)$ (related to yet another decomposition of $u(t, x)$ which requires the decay $(2.72)$ ), with $\bar{\lambda}(t) \approx \tilde{\lambda}(t)$ such that the following holds, for a universal constant $c_{0}>0$,

$$
-E_{0} \lambda^{2} \lesssim \int \tilde{\varepsilon} Q<0, \quad\left|2 \int \tilde{\varepsilon} Q+c_{0} \bar{\lambda}^{2} \bar{\lambda}_{t}\right| \lesssim\left(\int \tilde{\varepsilon}^{2} e^{-\frac{|y|}{4}}\right)^{\frac{3}{4}} .
$$

Since $\int_{t_{2}}^{t_{1}} \overline{\lambda \lambda_{t}} d t \lesssim \bar{\lambda}^{2}\left(t_{1}\right) \lesssim \tilde{\lambda}^{2}\left(t_{1}\right)$, we obtain (2.75) by integration.

Passing to the limit as $t_{2} \rightarrow T$ in (2.75), we obtain

$$
\int_{T}^{t_{1}} \frac{\int \tilde{\varepsilon}^{2} e^{-\frac{|y|}{10^{4}}} d y}{\tilde{\lambda}^{3}} d t \lesssim \tilde{\lambda}^{2}\left(t_{1}\right) E_{0} \lesssim \lambda^{2}\left(t_{1}\right) E_{0},
$$

and thus, using (2.70),

$$
\int_{T}^{t_{1}} \frac{\int \varepsilon^{2} e^{-\frac{2|y|}{10^{4}}} d y}{\lambda^{3}} d t \lesssim \lambda^{2}\left(t_{1}\right) E_{0}
$$

By (2.30), we have $\frac{b^{2}}{\lambda^{3}} \leq-b_{t}+\frac{C}{\lambda^{3}} \int \varepsilon^{2} e^{-\frac{|y|}{10}} d y$ and thus

$$
\int_{T}^{t_{1}} \frac{b^{2}(t)}{\lambda^{3}(t)} d t \lesssim \lambda^{2}\left(t_{1}\right) E_{0}+C \int_{T}^{t_{1}} \frac{\int \varepsilon^{2} e^{-\frac{|y|}{10}}}{\lambda^{3}(t)} d y \lesssim \lambda^{2}\left(t_{1}\right) E_{0}
$$

Now, we claim

$$
\int_{y>1} y^{10} \varepsilon^{2}(t, y) d y \lesssim \lambda^{2}(t) E_{0}
$$

Indeed, consider a smooth function

$$
\varphi_{10}(y)=\left\{\begin{array}{ll}
0 & \text { for } y \leq 0, \\
y^{10} & \text { for } y \geq 1,
\end{array} \quad \text { with } \varphi_{10}^{\prime} \geq 0 .\right.
$$

Using the computations of the proof of Lemma 3.7 in [33] on $\tilde{\varepsilon}$ (the computations for the decomposition $(\tilde{\varepsilon}, \tilde{\lambda}, \tilde{x})$ are actually simpler, since they correspond to the choice $b=0)$, we obtain

$$
\frac{1}{\tilde{\lambda}^{10}} \frac{d}{d t}\left(\tilde{\lambda}^{10} \int \varphi_{10} \tilde{\varepsilon}^{2}\right) \lesssim \frac{\int \tilde{\varepsilon}^{2} e^{-\frac{|y|}{10}} d y}{\tilde{\lambda}^{3}}
$$

and thus, using (2.64), (2.78)

$$
\int \varphi_{10} \tilde{\varepsilon}^{2}\left(t_{1}, y\right) d y \lesssim \int \varphi_{10} \tilde{\varepsilon}^{2}\left(t_{2}, y\right) d y+\lambda^{2}\left(t_{1}\right) E_{0}
$$

Passing to the limit as $t_{2} \rightarrow T$ and using (2.74), we obtain for all $T<t<t_{0}$,

$$
\int_{y>1} y^{10} \tilde{\varepsilon}^{2}(t, y) d y \lesssim \lambda^{2}(t) E_{0}
$$

Finally, by (2.70) and (2.69), we obtain (2.80). 


\section{Construction of a minimal element}

This section is devoted to the proof of the existence of a minimal blow up element. We propose a strategy of proof slightly different from the recent approach developed for the construction of non dispersive solutions in [36, 25, 31, 5, 6, 7, 19, 53, 20, mainly to prepare the analysis of the (Exit) regime in Theorem [1.4, see also section A.2 and Remark 3.2 below.

The strategy of the proof goes as follows. We consider a well prepared sequence of initial data $\left(u_{n}\right)$ with

$$
\left\|u_{n}\right\|_{L^{2}}<\|Q\|_{L^{2}} \text { and } u_{n}(0) \rightarrow Q \text { in } H^{1} .
$$

By Theorem 1.1, such solutions are in the (Exit) scenario and we denote by $t_{n}^{*}>0$ the corresponding exit time. The estimates extracted from [33] allow for a complete dynamical description of the (Exit) regime and in particular the defocusing structure of the solution at $t_{n}^{*}$. This explicit detailed knowledge allows us to renormalize the flow and extract in the limit $n \rightarrow+\infty$ a solution $v \in \mathcal{C}\left(\left(t^{*}, 0\right], H^{1}\right)$ which blows up at time $t^{*}<0$ and has subcritical mass $\|v\|_{L^{2}} \leq\|Q\|_{L^{2}}$. But then the global wellposedness below the ground state mass implies $\|v\|_{L^{2}}=\|Q\|_{L^{2}}$ and $v$ is an $H^{1}$ minimal mass blow up element.

step 1 Well prepared data. Let $u_{n}(0)=Q_{b_{n}(0)}$, where $b_{n}(0)=-\frac{1}{n}$ so that

$$
u_{n}(0) \in \mathcal{A} \subset H^{1}, \quad u_{n}(0) \rightarrow Q \quad \text { in } H^{1} \text { as } n \rightarrow+\infty .
$$

By (2.16), we have $\int u_{n}^{2}(0)<\int Q^{2}$. In particular, from energy and mass conservation, and the Gagliardo-Nirenberg's inequality (1.3), the solution $u_{n}(t)$ is global. We take $n>0$ large enough and we apply Theorem 1.1. The solution $u_{n}$ being global, the (Blow up) scenario is ruled out. The solution cannot converge locally to a solitary wave because of mass conservation and the strictly subcritical mass assumption, hence (Soliton) is also ruled out. Hence (Exit) holds and we define the exit time (related to the constant $\alpha^{*}$ of Theorem 1.1) by

$$
t_{n}^{*}=\sup \left\{t>0, \text { such that } \forall t^{\prime} \in[0, t], u_{n}\left(t^{\prime}\right) \in \mathcal{T}_{\alpha^{*}}\right\} .
$$

Note that $t_{n}^{*} \rightarrow+\infty$ as $n \rightarrow+\infty$ from the continuous dependence of the solution of (1.1) with respect to the initial data, and the fact that $Q(x-t)$ is solution of (1.1).

Now, we use refined information given in the (Exit) case by Proposition 4.1 in 33. In particular, we know that $u_{n}(t)$ satisfies (2.19) and has a decomposition $\left(\lambda_{n}, x_{n}, b_{n}, \varepsilon_{n}\right)$ as in Lemma [2.6 on $\left[0, t_{n}^{*}\right]$. Moreover, (H1)-(H3) are satisfied on $\left[0, t_{n}^{*}\right]$, and by definition of $u_{n}(0)$,

$$
\lambda_{n}(0)=1, \quad x_{n}(0)=0, \quad b_{n}(0)=-\frac{1}{n}, \quad \varepsilon_{n}(0)=0 .
$$

In addition, from the proof of Proposition 4.1 (see (4.41) in [33]), we also have

$$
\text { for all } 0 \leq t_{1} \leq t_{2} \leq t_{n}^{*}, \quad \lambda_{n}\left(t_{2}\right) \geq \frac{1}{2} \lambda_{n}\left(t_{1}\right) .
$$

Note also that by continuity in time and the definition of $t_{n}^{*}$,

$$
\inf _{\lambda_{0}>0, x_{0} \in \mathbb{R}}\left\|u_{n}\left(t_{n}^{*}\right)-\lambda_{0}^{-1 / 2} Q\left(\lambda_{0}^{-1}\left(.+x_{0}\right)\right)\right\|_{L^{2}}=\alpha^{*},
$$

and

$$
\alpha^{*} \leq \| u_{n}\left(t_{n}^{*}\right)-\lambda_{n}^{-1 / 2}\left(t_{n}^{*}\right) Q\left(\lambda_{n}^{-1}\left(t_{n}^{*}\right)\left(.+x_{n}\left(t_{n}^{*}\right)\right) \|_{L^{2}} \leq \delta\left(\alpha^{*}\right) .\right.
$$


step 2 Structure of the defocalized bubble. From Lemma 2.10, (3.1) and (3.4), we claim:

\section{Lemma 3.1.}

(i) Estimates on $\left[0, t_{n}^{*}\right]$.

$$
\begin{aligned}
\forall t \in\left[0, t_{n}^{*}\right], \quad \frac{1-\delta\left(\alpha^{*}\right)}{n} & \leq-\frac{b_{n}(t)}{\lambda_{n}^{2}(t)} \leq \frac{1+\delta\left(\alpha^{*}\right)}{n}, \\
\left\|\varepsilon_{n}(t)\right\|_{H^{1}}^{2} & \lesssim \frac{\lambda_{n}^{2}(t)}{n} \lesssim \delta\left(\alpha^{*}\right) .
\end{aligned}
$$

(ii) Estimates at $t_{n}^{*}$. For all $n$,

$$
\begin{gathered}
\left(\alpha^{*}\right)^{2} \lesssim-b_{n}\left(t_{n}^{*}\right) \lesssim \delta\left(\alpha^{*}\right), \\
\left(\alpha^{*}\right)^{2} \lesssim \int \varepsilon_{n}^{2}\left(t_{n}^{*}\right) \lesssim \delta\left(\alpha^{*}\right), \quad\left(\alpha^{*}\right)^{2} \lesssim \frac{\lambda_{n}^{2}\left(t_{n}^{*}\right)}{n} \lesssim \delta\left(\alpha^{*}\right), \\
0<c\left(\alpha^{*}\right) \leq \frac{t_{n}^{*}}{\lambda_{n}^{3}\left(t_{n}^{*}\right)} \leq C\left(\alpha^{*}\right) .
\end{gathered}
$$

(iii) Control of the dynamics on $\left[0, t_{n}^{*}\right]$.

$$
-\left(1-\delta\left(\alpha^{*}\right)\right) \frac{b_{n}\left(t_{n}^{*}\right)}{\lambda_{n}^{2}\left(t_{n}^{*}\right)} \leq\left(\lambda_{0 n}\right)_{t}(t) \leq-\left(1+\delta\left(\alpha^{*}\right)\right) \frac{b_{n}\left(t_{n}^{*}\right)}{\lambda_{n}^{2}\left(t_{n}^{*}\right)} .
$$

Proof. Using (2.33) and (2.35) at $t=0$, we obtain

$$
\begin{gathered}
\int u_{n}^{2}(0)=\int Q^{2}-\frac{2}{n} \int P Q+O\left(n^{-9 / 8}\right), \\
2 E\left(u_{n}(0)\right)=\frac{2}{n} \int P Q+O\left(n^{-9 / 8}\right) .
\end{gathered}
$$

Combining the conservation of the $L^{2}$ norm, the conservation of energy and (2.33), (2.35), we obtain at any $t \in\left(0, t_{n}^{*}\right)$,

$$
\left|b_{n}\right| \lesssim \int \varepsilon^{2}+\frac{1}{n}
$$

and

$$
\left(L \varepsilon_{n}(t), \varepsilon_{n}(t)\right)=2 \lambda_{n}^{2} E\left(u_{n}(0)\right)+\int u_{n}^{2}(0)-\int Q^{2}+O\left(n^{-\frac{9}{8}}+\left\|\varepsilon_{n}(t)\right\|_{H^{1}}^{\frac{9}{4}}\right),
$$

Thus, by $\left\|\varepsilon_{n}(t)\right\|_{H^{1}}^{2} \lesssim\left(L \varepsilon_{n}(t), \varepsilon_{n}(t)\right)$, we obtain

$$
\left\|\varepsilon_{n}(t)\right\|_{H^{1}}^{2} \lesssim \frac{\lambda_{n}^{2}(t)}{n}
$$

Next, recall (3.4)

$$
\alpha^{*} \leq\left\|b_{n}\left(t_{n}^{*}\right) \chi_{b_{n}\left(t_{n}^{*}\right)} P+\varepsilon_{n}\left(t_{n}^{*}\right)\right\|_{L^{2}} \leq \delta\left(\alpha^{*}\right),
$$

and use (2.32) and (3.11) to obtain

$$
\left(\alpha^{*}\right)^{2} \lesssim \int \varepsilon_{n}^{2}\left(t_{n}^{*}\right) \lesssim \delta\left(\alpha^{*}\right), \quad\left(\alpha^{*}\right)^{2} \lesssim-b_{n}\left(t_{n}^{*}\right) \lesssim \delta\left(\alpha^{*}\right),
$$

Now, we use the dynamical information given by the rigidity property (2.57) and the initialization (3.1): for all $t \in\left[0, t_{n}^{*}\right]$,

$$
\left(1+\delta\left(\alpha^{*}\right)\right) b_{n}(0) \leq \frac{b_{n}(t)}{\lambda_{n}^{2}(t)} \leq\left(1-\delta\left(\alpha^{*}\right)\right) b_{n}(0)
$$


and thus by (3.13),

$$
\left(\alpha^{*}\right)^{2} \lesssim \frac{\lambda_{n}^{2}\left(t_{n}^{*}\right)}{n} \lesssim \delta\left(\alpha^{*}\right) .
$$

Finally, let us prove (3.10) and (3.9). By (2.58), we have

$$
\left|\left(\lambda_{0 n}\right)_{t} \frac{\lambda_{n}}{\lambda_{0 n}}+\frac{b_{n}}{\lambda_{n}^{2}}\right| \lesssim \frac{\int \varepsilon_{n}^{2} e^{-\frac{|y|}{10}}}{\lambda_{n}^{2}}+\frac{\left|b_{n}\right|}{\lambda_{n}^{2}}\left(\mathcal{N}_{n}^{\frac{1}{2}}+\left|b_{n}\right|\right) \lesssim \frac{1}{\lambda_{n}^{2}}\left(\mathcal{N}_{n}+\left|b_{n}\right|^{2}\right) .
$$

By (2.60), (3.1), and then (3.14), we have

$$
\frac{\mathcal{N}_{n}(t)}{\lambda_{n}^{2}(t)} \lesssim \frac{\left|b_{n}(t)\right|^{3}}{\lambda_{n}^{2}(t)}+\frac{\left|b_{n}(0)\right|^{3}}{\lambda_{n}^{2}(0)} \lesssim \delta\left(\alpha^{*}\right) \frac{\left|b_{n}\left(t_{n}^{*}\right)\right|}{\lambda_{n}^{2}\left(t_{n}^{*}\right)} .
$$

Thus, again by (3.14),

$$
-\left(1-\delta\left(\alpha^{*}\right)\right) \frac{b_{n}\left(t_{n}^{*}\right)}{\lambda_{n}^{2}\left(t_{n}^{*}\right)} \leq\left(\lambda_{0 n}\right)_{t}(t) \leq-\left(1+\delta\left(\alpha^{*}\right)\right) \frac{b_{n}\left(t_{n}^{*}\right)}{\lambda_{n}^{2}\left(t_{n}^{*}\right)},
$$

which is (3.10). We integrate on $\left[0, t_{n}^{*}\right]$ and then divide by $\lambda_{0, n}\left(t_{n}^{*}\right)$ to obtain:

$$
-t_{n}^{*} \frac{b_{n}\left(t_{n}^{*}\right)}{\lambda_{n}^{3}\left(t_{n}^{*}\right)}\left(1-\delta\left(\alpha^{*}\right)\right) \leq 1-\frac{1}{\lambda_{0, n}\left(t_{n}^{*}\right)} \leq-t_{n}^{*} \frac{b_{n}\left(t_{n}^{*}\right)}{\lambda_{n}^{3}\left(t_{n}^{*}\right)}\left(1+\delta\left(\alpha^{*}\right)\right) .
$$

Hence using (3.15) and $\frac{\lambda_{n}}{\lambda_{0, n}} \lesssim \mathcal{N}_{n}^{\frac{1}{2}} \lesssim \delta\left(\alpha^{*}\right)$, we obtain

$$
-\frac{1-\delta\left(\alpha^{*}\right)}{b_{n}\left(t_{n}^{*}\right)} \leq \frac{t_{n}^{*}}{\lambda_{n}^{3}\left(t_{n}^{*}\right)} \leq-\frac{1+\delta\left(\alpha^{*}\right)}{b_{n}\left(t_{n}^{*}\right)},
$$

which together with (3.13) implies (3.9).

step 3 Renormalization and extraction of the limit. Let:

$$
\begin{aligned}
& \forall \tau \in\left[-\frac{t_{n}^{*}}{\lambda_{n}^{3}\left(t_{n}^{*}\right)}, 0\right], \quad t_{\tau}=t_{n}^{*}+\tau \lambda_{n}^{3}\left(t_{n}^{*}\right), \\
v_{n}(\tau, x) \quad= & \lambda_{n}^{\frac{1}{2}}\left(t_{n}^{*}\right) u_{n}\left(t_{\tau}, \lambda_{n}\left(t_{n}^{*}\right) x+x\left(t_{n}^{*}\right)\right) \\
= & \frac{\lambda_{n}^{\frac{1}{2}}\left(t_{n}^{*}\right)}{\lambda_{n}^{\frac{1}{2}}\left(t_{\tau}\right)}\left(Q_{b_{n}\left(t_{\tau}\right)}+\varepsilon_{n}\right)\left(t_{\tau}, \frac{\lambda_{n}\left(t_{n}^{*}\right)}{\lambda_{n}\left(t_{\tau}\right)} x+\frac{x\left(t_{n}^{*}\right)-x\left(t_{\tau}\right)}{\lambda_{n}\left(t_{\tau}\right)}\right),
\end{aligned}
$$

so that $v_{n}$ is solution of (1.1) and belongs to the tube $\mathcal{T}_{\alpha^{*}}$ for $\tau \in\left[-\frac{t_{n}^{*}}{\lambda_{n}^{3}\left(t_{n}^{*}\right)}, 0\right]$. Moreover, its decomposition $\left(\lambda_{v_{n}}, x_{v_{n}}, \varepsilon_{v_{n}}\right)$ satisfies on $\left[-\frac{t_{n}^{*}}{\lambda_{n}^{3}\left(t_{n}^{*}\right)}, 0\right]$

$$
\lambda_{v_{n}}(\tau)=\frac{\lambda_{n}\left(t_{\tau}\right)}{\lambda_{n}\left(t_{n}^{*}\right)}, x_{v_{n}}(\tau)=\frac{x_{n}\left(t_{\tau}\right)-x_{n}\left(t_{n}^{*}\right)}{\lambda_{n}\left(t_{n}^{*}\right)}, b_{v_{n}}(\tau)=b_{n}\left(t_{\tau}\right), \varepsilon_{v_{n}}(\tau)=\varepsilon_{n}\left(t_{\tau}\right) .
$$

By (3.7), (3.8) and (3.5), we have

$$
\begin{gathered}
\forall \tau \in\left[-\frac{t_{n}^{*}}{\lambda_{n}^{3}\left(t_{n}^{*}\right)}, 0\right], \quad\left\|\varepsilon_{v_{n}}(\tau)\right\|_{H^{1}}^{2} \lesssim \delta\left(\alpha^{*}\right) . \\
\lambda_{v_{n}}(0)=1, \quad x_{v_{n}}(0)=0, \quad\left(\alpha^{*}\right)^{2} \lesssim-b_{v_{n}}(0) \leq \delta\left(\alpha^{*}\right) .
\end{gathered}
$$

Therefore, there exists a subsequence of $\left(v_{n}\right)$, which we will still denote by $\left(v_{n}\right)$, and $v(0) \in H^{1}$ such that

$$
v_{n}(0) \rightarrow v(0) \text { in } H^{1} \text { weak, and }\|v(0)-Q\|_{H^{1}} \lesssim \delta\left(\alpha^{*}\right) .
$$


and, by (3.8) and (3.9),

$$
\tau_{n}^{*}=-\frac{t_{n}^{*}}{\lambda_{n}^{3}\left(t_{n}^{*}\right)} \rightarrow-\tau^{*}, \quad \tau^{*}>0, \quad-b_{n}\left(t_{n}^{*}\right) \rightarrow b^{*}>0 .
$$

Moreover, by (3.17),

$$
\frac{1-\delta\left(\alpha^{*}\right)}{b^{*}} \leq \tau^{*} \leq \frac{1+\delta\left(\alpha^{*}\right)}{b^{*}} .
$$

We let $v(\tau)$ be the backward $H^{1}$ solution of (1.1) with initial data $v(0)$ at $\tau=0$.

step 4 Minimal mass blow up. We claim that $v$ is a minimal mass blow up element $\|v\|_{L^{2}}=\|Q\|_{L^{2}}$ which blows up in finite negative time $-\tau^{*}$ with for $\tau$ close enough to $-\tau^{*}$ :

$$
\frac{\left(1-\delta\left(\alpha^{*}\right)\right)}{1+\frac{\tau}{\tau^{*}}} \leq\left\|v_{x}(\tau)\right\|_{L^{2}} \leq \frac{\left(1+\delta\left(\alpha^{*}\right)\right)}{1+\frac{\tau}{\tau^{*}}} .
$$

Indeed, we integrate (3.10) and obtain for $t \in\left[0, t_{n}^{*}\right], n$ large enough,

$$
\frac{b^{*} t}{\lambda_{n}^{2}\left(t_{n}^{*}\right)}\left(1-\delta\left(\alpha^{*}\right)\right) \leq \lambda_{0, n}(t)-\lambda_{0, n}(0) \leq \frac{b^{*} t}{\lambda_{n}^{2}\left(t_{n}^{*}\right)}\left(1+\delta\left(\alpha^{*}\right)\right) .
$$

We conclude from (3.20) and the definition of $\tau_{n}^{*}$ : for all $\tau \in\left[\tau_{n}^{*}, 0\right]$,

$$
b^{*}\left(\tau_{n}^{*}+\tau\right)\left(1-\delta\left(\alpha^{*}\right)\right) \leq \lambda_{0, v_{n}}(\tau)-\lambda_{0, v_{n}}\left(\tau_{n}^{*}\right) \leq b^{*}\left(\tau_{n}^{*}+\tau\right)\left(1+\delta\left(\alpha^{*}\right)\right) .
$$

Let $\tau_{0} \in\left(-\tau^{*}, 0\right)$. From (3.8), (3.20) and $\varepsilon_{v_{n}}\left(\tau_{n}^{*}\right)=\varepsilon_{n}(0)$, we have

$$
\lambda_{0, v_{n}}\left(\tau_{n}^{*}\right)=\frac{\lambda_{n}(0)}{\lambda_{n}\left(t_{n}^{*}\right)} \rightarrow 0 \text { as } n \rightarrow+\infty
$$

and $\left(1-\delta\left(\alpha^{*}\right)\right) \leq \frac{\lambda_{0, v_{n}}}{\lambda_{v_{n}}} \leq\left(1+\delta\left(\alpha^{*}\right)\right)$. Thus, we conclude from (3.23) that for $n$ large enough depending on $\tau_{0}$,

$$
\forall \tau \in\left[\tau_{0}, 0\right], \quad b^{*}\left(\tau^{*}+\tau\right)\left(1-\delta\left(\alpha^{*}\right)\right) \leq \lambda_{v_{n}}(\tau) \leq b^{*}\left(\tau^{*}+\tau\right)\left(1+\delta\left(\alpha^{*}\right)\right),
$$

and

$$
\frac{1}{2} b^{*}\left(\tau^{*}+\tau_{0}\right) \leq \lambda_{v_{n}}(\tau) .
$$

It follows from Lemma 2.8 that $v(\tau)$ is well-defined and $\lambda_{v_{n}}(\tau) \rightarrow \lambda_{v}(\tau)$ on $\left[\tau_{0}, 0\right]$. In particular, $v$ exists on $\left(-\tau^{*}, 0\right]$ and for all $\tau \in\left(-\tau^{*}, 0\right]$,

$$
b^{*}\left(\tau^{*}+\tau\right)\left(1-\delta\left(\alpha^{*}\right)\right) \leq \lambda_{v}(\tau) \leq b^{*}\left(\tau^{*}+\tau\right)\left(1+\delta\left(\alpha^{*}\right)\right),
$$

which together with (3.21) implies (3.22). Finally, we have by weak $H^{1}$ convergence $\int v^{2}(0) \leq \lim _{n \rightarrow \infty} \int v_{n}^{2}(0)=\int Q^{2}$, and since $v$ blows up in finite time, $\int v^{2}(0)=$ $\int Q^{2}$.

This concludes the proof of the existence of the minimal element.

Remark 3.2. We may rewrite this proof by saying that understanding the minimal mass blow up scenario is in some sense equivalent to understanding how subcritical solutions initially near the ground state move away from the ground state and start defocusing, and here the sharp knowledge of the speed of defocusing is fundamental for the proof. Another approach for the construction of the minimal blow up element in the continuation of $36,25,31,5,6,7,19,53$ would have been to take the initial data $Q_{b\left(t_{n}\right)}$ at some time $t_{n} \downarrow 0$ with $b\left(t_{n}\right)=t_{n}$ and to obtain uniform $H^{1}$ bounds on the corresponding forward solution $u_{n}(t)$ to (1.1) at a time $t_{0}>0$ independent of $n$ using the monotonicity machinery of Proposition 2.9 and Lemma 2.10, It is not clear to us whether a direct fixed point approach as in [3, 23, 24, 10, 11] is applicable here due to the poor localization in space of the minimal element. 


\section{Sharp description of minimal mass blow up}

We now turn to the proof of uniqueness in $H^{1}$ of the minimal element. Let us stress the fact that uniqueness is always a delicate problem, in particular in the absence of suitable symmetries as in [31]. As in [53], the first crucial information is to derive the blow up speed for all minimal elements, and here we shall use the a priori localization in space of minimal elements given by Lemma 2.11 which allows us to use the monotonicity tools Proposition 2.9 and Lemma 2.10. Once the minimal mass blow up regime is sufficiently well described, we may rerun the analysis of Proposition 2.9 for the difference of two such bubbles and conclude that they are equal, this is done in section 5 .

4.1. Finite time blow up and blow up speed for minimal mass blow up solutions. Our aim in this section is to derive sharp qualitative bounds on minimal mass blow up solutions, improving general results stated in Lemma 2.11. In particular, we prove that the blow up time is finite, $T>-\infty$, and we specify the blow up speed and the behavior of the concentration point which are essential preliminary information on the singularity formation. Note that the additional information below requires the sharper analysis of [33] and cannot be derived from [30]. We consider $u(t)$ a minimal mass blow up solution and in the setting of Lemma 2.11, we introduce the rescaled time

$$
s(t)=-\int_{t}^{t_{0}} \frac{d s}{\lambda^{3}} .
$$

Recall that $s(T)=-\infty$ from a standard argument (see e.g. [28]).

Proposition 4.1 (Sharp bounds). Let $u(t)$ be a solution of (1.1) defined on (T,0], which blows up backwards in finite or infinite time $-\infty \leq T<0$. Assume

$$
\int u^{2}(0)=\int Q^{2}
$$

(i) Finite time blow up: There holds

$$
T>-\infty
$$

(ii) Sharp controls near blow up time: there exist universal constants $c_{\lambda}, c_{x}, c_{b}$ and $\ell^{*}=\ell^{*}(u)<0, x^{*}=x^{*}(u) \in \mathbb{R}$ such that, for $t$ close to $T$,

$$
\begin{gathered}
\lambda(t)=\left|\ell^{*}\right|(t-T)+c_{\lambda}\left|\ell^{*}\right|^{4}(t-T)^{3}+O\left[(t-T)^{4}\right], \\
x(t)=-\frac{1}{\left(\ell^{*}\right)^{2}(t-T)}+x^{*}+c_{x} \ell^{*}(t-T)+O\left[(t-T)^{2}\right], \\
\frac{b(t)}{\lambda^{2}(t)}=\ell^{*}+c_{b}\left(\ell^{*}\right)^{4}(t-T)^{2}+O\left[(t-T)^{3}\right], \\
\mathcal{N}(t) \lesssim(t-T)^{6} .
\end{gathered}
$$

(iii) Estimates in rescaled time: for $-s$ large,

$$
\begin{gathered}
\|\varepsilon(s)\|_{L^{\infty}}^{2} \lesssim\|\varepsilon(s)\|_{H^{1}}^{2} \lesssim \lambda^{2}(s) \lesssim \frac{1}{|s|}, \\
\mathcal{N}(s)+\int_{-\infty}^{s} \int\left(\varepsilon_{y}^{2}+\varepsilon^{2}\right)\left(s^{\prime}\right) \varphi_{B}^{\prime} d s^{\prime} \lesssim \frac{1}{|s|^{3}}, \\
\left|\frac{\lambda_{s}}{\lambda}+b\right|+\left|\frac{x_{s}}{\lambda}-1\right| \lesssim \frac{1}{|s|^{\frac{3}{2}}}, \quad\left|b_{s}\right| \lesssim \frac{1}{|s|^{2}}, \\
b(s)=\frac{1}{2 s}+\frac{c_{1}^{*} \log |s|}{s^{2}}+\frac{c_{2}^{*}}{s^{2}}+o\left(\frac{1}{s^{2}}\right) \text { as } s \rightarrow-\infty
\end{gathered}
$$


for some universal constants $\left(c_{1}^{*}, c_{2}^{*}\right) \in \mathbb{R} \times \mathbb{R}$.

(iv) Global forward behavior: the solution is globally defined for $t>T, u \in$ $\mathcal{C}((0,+\infty) \times \mathbb{R})$ and for some $C(t), \gamma(t)>0$,

$$
\forall t>T, \forall x>0, \quad|u(t, x)| \leq C(t) e^{-\gamma(t) x} .
$$

(v) Time decay of weighted Sobolev norms: For $|s|$ large,

$$
\int \varepsilon^{2}(s, y) e^{\lambda(s) y} d y \lesssim \frac{1}{|s|^{2}} .
$$

For all $\frac{9}{B} \leq \omega \leq \frac{1}{10}$, for $|s|$ large,

$$
\begin{gathered}
\sum_{k=0}^{3} \int\left(\partial_{y}^{k} \varepsilon\right)^{2}(s, y) e^{\omega y} d y+\int_{-\infty}^{s} \sum_{k=0}^{4} \int\left(\partial_{y}^{k} \varepsilon\right)^{2}\left(s^{\prime}, y\right) e^{\omega y} d y d s^{\prime} \lesssim \frac{1}{|s|}, \\
\left\|\left(\left(\varepsilon_{i}\right)_{y y}^{2}+\left(\varepsilon_{i}\right)_{y}^{2}\right)(s) e^{\omega y}\right\|_{L^{\infty}} \lesssim \frac{1}{|s|} .
\end{gathered}
$$

Remark 4.2. The constant $\ell^{*}$ in (4.2) depends on the solution and the scaling $u(t, x) \mapsto u_{\lambda_{0}}(t, x)=\lambda_{0}^{\frac{1}{2}} u\left(\lambda_{0}^{3} t, \lambda_{0} x\right)$ leads to

$$
\ell^{*}\left(u_{\lambda_{0}}\right)=\lambda_{0}^{2} \ell^{*}(u) .
$$

Proof of Proposition 4.1. From Lemma 2.11, $E(u(t))>0$. Using the scaling invariance of the $(\mathrm{gKdV})$ equation, we consider the solution

$$
u_{\lambda_{0}}(t, x)=\lambda_{0}^{\frac{1}{2}} u\left(\lambda_{0}^{3} t, \lambda_{0} x\right),
$$

where $\lambda_{0}>0$ is chosen so that $E\left(u_{\lambda_{0}}(t)\right) \ll \kappa^{*}, \kappa^{*}$ being the small constant in Proposition 2.9. We work on $u_{\lambda_{0}}$ instead of working on $u$, all statement being scaling invariant. Hereafter, we denote $u_{\lambda_{0}}$ simply by $u(t)$.

step 1 Entering the monotonicity regime. Note first that from Lemma 2.11 and $E_{0} \ll \kappa^{*}$, (H1), (H2), (H3) hold on $\left(-\infty, s_{0}\right]$, for $-s_{0}$ large enough. The solution is therefore in the monotonicity regime of Proposition 2.9 and Lemma 2.10 on $\left(-\infty, s_{0}\right]$.

step 2 Rigidity and blow up speed. We claim the key non degeneracy relation:

$$
\mathcal{N} \lesssim O\left(\lambda^{6}\right), \quad\left|\frac{b}{\lambda^{2}}-\ell^{*}-\frac{c_{0}}{2} \frac{b^{2}}{\lambda^{2}}\right| \lesssim O\left(\lambda^{3}\right)
$$

for some constant $\ell^{*}>0$.

Let $C^{*}>0$ be the universal constant in (2.57). Let us first remark that there exists a sequence $s_{n} \rightarrow-\infty$ such that

$$
\forall n \geq 1, \quad b\left(s_{n}\right) \leq-C^{*} \int\left(\varepsilon_{y}^{2}+\varepsilon^{2}\right)\left(s_{n}\right) \varphi_{B}^{\prime} .
$$

Indeed, assume for the sake of contradiction that there exists a time $s^{*} \leq s_{0}$ such that (recall that $b<0$ )

$$
\forall s<s^{*}, \quad|b(s)| \leq C^{*} \int\left(\varepsilon_{y}^{2}+\varepsilon^{2}\right)(s) \varphi_{B}^{\prime} .
$$

Thus, (2.58) implies

$$
\left|\frac{\left(\lambda_{0}\right)_{s}}{\lambda_{0}}\right| \lesssim \int \varepsilon^{2} e^{-\frac{|y|}{10}}+|b| \lesssim \int\left(\varepsilon_{y}^{2}+\varepsilon^{2}\right)(s) \varphi_{B}^{\prime},
$$


where $\lambda_{0}(s)=\lambda(s)\left(1-J_{1}(s)\right)^{2}$. Using (2.59), we obtain

$$
\forall s<s_{2}^{*}, \quad\left|\log \left(\frac{\lambda_{0}(s)}{\lambda_{0}\left(s_{2}^{*}\right)}\right)\right| \lesssim \int_{s}^{s^{*}} \int\left(\varepsilon_{y}^{2}+\varepsilon^{2}\right)(s) \varphi_{B}^{\prime} d s \lesssim 1
$$

but together with

$$
\left|\frac{\lambda}{\lambda_{0}}-1\right| \lesssim\left|J_{1}\right| \lesssim \mathcal{N}^{\frac{1}{2}}
$$

and (2.63), this contradicts the blow up assumption:

$$
\lambda(s) \rightarrow 0 \text { as } s \rightarrow-\infty .
$$

The sign $b<0$ concludes the proof of (4.16).

Inserting (4.16) in (2.57) yields the rigidity:

$$
\text { for all } n \geq 1 \text {, for all } s \text { such that } s_{n} \leq s \leq s_{0}, 2 \frac{b\left(s_{n}\right)}{\lambda^{2}\left(s_{n}\right)} \leq \frac{b(s)}{\lambda^{2}(s)} \leq \frac{b\left(s_{n}\right)}{2 \lambda^{2}\left(s_{n}\right)} \text {. }
$$

We conclude using $b<0$ and $\lambda\left(s_{0}\right)=1$ that for all $s<s_{0}$,

$$
4 b\left(s_{0}\right) \leq \frac{b(s)}{\lambda^{2}(s)} \leq \frac{b\left(s_{0}\right)}{4}<0 .
$$

By (2.62) and (2.63), we have $\lim _{-\infty} \mathcal{N}=0$. Using (2.59), we have, for $s_{1}<s_{2}$

$$
\mathcal{N}\left(s_{2}\right)+\int_{s_{1}}^{s_{2}} \int\left(\varepsilon_{y}^{2}+\varepsilon^{2}\right) \varphi_{B}^{\prime} d s \lesssim \mathcal{N}\left(s_{1}\right)+\left|b^{3}\left(s_{1}\right)\right|+\left|b^{3}\left(s_{2}\right)\right| .
$$

Thus, passing to the limit $s_{1} \rightarrow-\infty$, and using (2.69),

$$
\mathcal{N}\left(s_{2}\right)+\int_{-\infty}^{s_{2}} \int\left(\varepsilon_{y}^{2}+\varepsilon^{2}\right) \varphi_{B}^{\prime} d s \lesssim\left|b^{3}\left(s_{2}\right)\right| \lesssim \lambda^{6}\left(s_{2}\right)
$$

From (2.41), we have

$$
\left|\frac{d}{d s}\left(\frac{b}{\lambda^{2}} e^{J}\right)+c_{0} \frac{b^{3}}{\lambda^{2}}\right| \leq \frac{1}{\lambda^{2}}\left(\int \varepsilon^{2} e^{-\frac{|y|}{10}}+|b|^{4}\right) .
$$

Letting $s_{1} \rightarrow-\infty$ in (2.60) ensures:

$$
\int_{-\infty}^{s_{2}} \frac{1}{\lambda^{2}}\left(\int \varepsilon^{2} e^{-\frac{|y|}{10}}+|b|^{4}\right) d s \lesssim \frac{b^{3}}{\lambda^{2}} \lesssim \lambda^{4}
$$

Next, by (2.30), (4.22):

$$
\frac{b^{3}}{\lambda^{2}}=-\frac{1}{2} \frac{b_{s} b}{\lambda^{2}}+O\left(\frac{1}{\lambda^{2}}\left(\int \varepsilon^{2} e^{-\frac{|y|}{10}}+|b|^{4}\right)\right),
$$

so that by integration by parts and (2.29),

$$
\int_{-\infty}^{s} \frac{b^{3}}{\lambda^{2}}=-\frac{1}{4} \frac{b^{2}(s)}{\lambda^{2}(s)}-\frac{1}{2} \int_{-\infty}^{s} \frac{b^{2} \lambda_{s}}{\lambda^{3}}+O\left(\lambda^{4}\right)=-\frac{1}{4} \frac{b^{2}(s)}{\lambda^{2}(s)}+\frac{1}{2} \int_{-\infty}^{s} \frac{b^{3}}{\lambda^{2}}+O\left(\lambda^{4}\right)
$$

and thus

$$
\int_{-\infty}^{s} \frac{b^{3}}{\lambda^{2}}=-\frac{1}{2} \frac{b^{2}(s)}{\lambda^{2}(s)}+O\left(\lambda^{4}\right) .
$$

It follows by integrating (4.21) and using (4.19) that

$$
\lim _{s \rightarrow-\infty} \frac{b}{\lambda^{2}}(s)=\ell^{*}<0,
$$


and more precisely, using $|J| \lesssim \mathcal{N}^{\frac{1}{2}} \lesssim \lambda^{3}$,

$$
\left|\frac{b}{\lambda^{2}}-\ell^{*}-\frac{c_{0}}{2} \frac{b^{2}}{\lambda^{2}}\right| \lesssim O\left(\lambda^{4}\right)+\frac{|b|}{\lambda^{2}}\left(1-e^{J}\right) \lesssim O\left(\lambda^{3}\right) .
$$

step 3 Finite time blow-up.

From (4.20) and (2.54), we have

$$
\lambda_{0}=\lambda+O\left(\lambda J_{1}\right)=\lambda+O\left(\lambda \mathcal{N}^{\frac{1}{2}}\right)=\lambda+O\left(\lambda^{4}\right)
$$

and then by (2.58), (4.24),

$$
\begin{aligned}
-\lambda^{3} \frac{\left(\lambda_{0}\right)_{t}}{\lambda_{0}} & =-\frac{\left(\lambda_{0}\right)_{s}}{\lambda_{0}}=b+c_{1} b^{2}+O\left(\mathcal{N}+|b| \mathcal{N}^{\frac{1}{2}}+|b|^{3}\right) \\
& =\ell^{*} \lambda^{2}+c\left(\ell^{*}\right)^{2} \lambda^{4}+O\left(\lambda^{5}\right),
\end{aligned}
$$

where $c$ denotes here and thereafter various universal constants. Hence

$$
-\left(\lambda_{0}\right)_{t}=\ell^{*}+c\left(\ell^{*}\right)^{2} \lambda_{0}^{2}+O\left(\lambda_{0}^{3}\right) .
$$

For $t$ close to $T$, we obtain $\left(\lambda_{0}\right)_{t}>\frac{\ell^{*}}{2}>0$ and thus $\lambda_{0}$ vanishes backwards at some finite time

$$
T>-\infty
$$

in particular, the solution blows up in finite time. Moreover, integrating (4.26) on $(T, t]$ for $t>T$ close to $T$, using (4.25), yields

$$
\lambda(t)=\lambda_{0}(t)+O\left[(t-T)^{4}\right]=\left|\ell^{*}\right|(t-T)+c\left|\ell^{*}\right|^{4}(t-T)^{3}+O\left[(t-T)^{4}\right] .
$$

Together with (4.20), (4.15), this concludes the proof of (4.4), (4.2), (4.5).

We now integrate the modulation equation (2.29) for the blow up point:

$$
x_{t}=\frac{1}{\lambda^{2}} \frac{x_{s}}{\lambda}=\frac{1}{\lambda^{2}}\left[1+O\left(b^{2}+\mathcal{N}^{\frac{1}{2}}\right)\right]=\frac{1}{\lambda^{2}}\left[1+O\left(\lambda^{3}\right)\right]
$$

and thus using (4.2):

$$
x_{t}(t)=\frac{1}{\left(\ell^{*}\right)^{2}(T-t)^{2}}-2 c \ell^{*}+O(t-T)
$$

which implies (4.3) by integration in time.

step 4 Sharp estimates in rescaled time. From (4.2):

$$
s(t)=-\int_{t}^{t_{0}} \frac{d t}{\left|\ell^{*}\right|^{3}(t-T)^{3}\left(1+O\left((T-t)^{2}\right)\right)}=-\frac{1}{2\left|\ell^{*}\right|^{3}(t-T)^{2}}(1+O(t-T)) .
$$

From step 3 and (2.29)-(2.30), we thus get the following estimates in terms of the variable $s$ :

$$
\begin{gathered}
\lambda(s)=\frac{1+o(1)}{\sqrt{2\left|\ell^{*} s\right|}}, \quad \mathcal{N}(s)+\int_{-\infty}^{s} \int\left(\varepsilon_{y}^{2}+\varepsilon^{2}\right) \varphi_{B}^{\prime} d s^{\prime} \lesssim \frac{1}{|s|^{3}}, \quad b(s)=\frac{1+o(1)}{2 s} \\
\left|\frac{\lambda_{s}}{\lambda}(s)\right| \lesssim|b(s)|+\mathcal{N}^{\frac{1}{2}}(s) \lesssim \frac{1}{s}, \quad\left|\frac{\lambda_{s}}{\lambda}+b\right|+\left|\frac{x_{s}}{\lambda}-1\right| \lesssim \frac{1}{|s|^{\frac{3}{2}}}
\end{gathered}
$$

and $\left|b_{s}\right| \lesssim \frac{1}{|s|^{2}}$, so that (4.6) and (4.8) are proved.

Now, we prove (4.9). We rewrite the sharp modulation equation (2.40) for $b$ as:

$$
\left|\left(b\left(1+J_{2}\right)\right)_{s}+2 b^{2}+c_{2} b^{3}\right| \lesssim \int\left(\varepsilon_{y}^{2}+\varepsilon^{2}\right) \varphi_{B}^{\prime}+\frac{1}{s^{4}}+\frac{1}{s^{2}} \mathcal{N}^{\frac{1}{2}} \lesssim \int\left(\varepsilon_{y}^{2}+\varepsilon^{2}\right) \varphi_{B}^{\prime}+\frac{1}{|s|^{\frac{7}{2}}} .
$$


Let

$$
\tilde{b}=b\left(1+J_{2}\right)=b+O\left(\frac{1}{|s|^{\frac{5}{2}}}\right)
$$

then equivalently:

$$
\left|\tilde{b}_{s}+2 \tilde{b}^{2}+c_{2} \tilde{b}^{3}\right| \lesssim \int\left(\varepsilon_{y}^{2}+\varepsilon^{2}\right) \varphi_{B}^{\prime}+\frac{1}{|s|^{\frac{7}{2}}}
$$

If $c_{2} \leq 1$, let $b_{0}=-1$, otherwise let $b_{0}=-1 / c_{2}$. In order to integrate this differential inequation, we let

$$
F(b)=\int_{b_{0}}^{b} \frac{d \beta}{2 \beta^{2}+c_{2} \beta^{3}}=-\frac{1}{2 b}-\frac{c_{2} \log |b|}{4}+c_{0}+O(b) \text { as } b \rightarrow 0,
$$

for some universal constant $c_{0} \in \mathbb{R}$. Then,

$$
\frac{d}{d s} F(\tilde{b})=-1+O\left(s^{2} \int\left(\varepsilon_{y}^{2}+\varepsilon^{2}\right) \varphi_{B}^{\prime}+\frac{1}{|s|^{\frac{3}{2}}}\right) .
$$

By (2.60) with $s_{1} \rightarrow-\infty$, we have

$$
s^{2} \mathcal{N}(s)+\int_{-\infty}^{s}\left(s^{\prime}\right)^{2} \int\left(\varepsilon_{y}^{2}+\varepsilon^{2}\right)\left(s^{\prime}\right) \varphi_{B}^{\prime} d s^{\prime} \lesssim \frac{1}{|s|} .
$$

Therefore, integrating (4.31) on $\left[s, s_{0}\right]$ and using (4.30):

$$
F(\tilde{b}(s))=-\frac{1}{2 \tilde{b}(s)}-\frac{c_{2} \log |\tilde{b}(s)|}{4}+c_{0}^{\prime}+O\left(\frac{1}{s}\right)=-s+O\left(\frac{1}{\sqrt{|s|}}\right)
$$

which is easily inverted to get:

$$
\tilde{b}(s)=\frac{1}{2 s}+\frac{c_{1}^{*} \log |s|}{s^{2}}+\frac{c_{2}^{*}}{s^{2}}+O\left(\frac{1}{|s|^{\frac{5}{2}}}\right)
$$

for some universal constants $c_{1}^{*}, c_{2}^{*}$. The estimate (4.29) now implies (4.9).

step 5 Global existence for $t>t_{0}$. Recall that for all $y>0,\left|\varepsilon\left(s_{0}, y\right)\right| \lesssim e^{-\frac{y}{20}}$. Thus, $u(t)$ has exponential decay in space on the right $(x>0)$, in particular, $\int_{x>0} x^{10} u^{2}\left(t_{0}\right)<\infty$. From this fact and since $u(t)$ has critical mass, we conclude from Theorem 1.2 that $u$ is globally defined for $t>t_{0}$. Since $Q$ has exponential decay at $\infty$, the exponential decay (2.65) obtained on $\varepsilon$ translates into exponential decay on $u$ (4.10). Finally, it is proved in [13] that a solution of $(\mathrm{gKdV})$ equation with such exponential decay on the right is smooth, i.e. $u \in C^{\infty}((0,+\infty) \times \mathbb{R})$.

The proofs of (4.11) and (4.12)-(4.13) are given in Appendix A.

4.2. Sharp description of $S(t)$. We conclude from Proposition 4.1 that the minimal element constructed in section 3 satisfies the following sharp bounds which conclude the proof of statements (i) and (iii) of Theorem 1.3.

Corollary 4.3. There exists a solution $S \in \mathcal{C}\left((0,+\infty), H^{1}\right) \cap \mathcal{C}^{\infty}((0,+\infty) \times \mathbb{R})$ to (1.1) with critical mass $\|S(t)\|_{L^{2}}=\|Q\|_{L^{2}}$ such that:

$$
\begin{gathered}
\left\|\partial_{x} S(t)\right\|_{L^{2}} \sim \frac{\left\|\partial_{x} Q\right\|_{L^{2}}}{t} \text { as } t \downarrow 0, \\
S(t, x)-\frac{1}{t^{\frac{1}{2}}} Q\left(\frac{x+\frac{1}{t}+\bar{c} t}{t}\right) \rightarrow 0 \text { in } L^{2} \text { as } t \downarrow 0, \\
\forall x>0, \quad|S(1, x)| \lesssim e^{-\gamma x}
\end{gathered}
$$


for some universal constants $(\bar{c}, \gamma) \in \mathbb{R} \times \mathbb{R}_{+}^{*}$. Moreover,

$$
\frac{d}{d t}\left(\inf _{\lambda_{1}>0, x_{1} \in \mathbb{R}}\left\|S(t)-Q_{\lambda_{1}}\left(.-x_{1}\right)\right\|_{L^{2}}^{2}\right)=4 t(P, Q)+O\left(t^{2}\right) .
$$

Proof of Corollary 4.3. Let $v(t, x)$ be the minimal mass blow up solution constructed in section 3 with finite backward blow up time $T<0$. Let $\ell^{*}=\ell^{*}(v)$ and $x^{*}=x^{*}(v)$ be the constants corresponding to $v$ in Proposition 4.1. From the invariances of the equation and Remark 4.2, $S(t)$ defined by

$$
S(t, x)=\left(\ell^{*}\right)^{-\frac{1}{4}} v\left(\left(\ell^{*}\right)^{-\frac{3}{2}} t+T,\left(\ell^{*}\right)^{-\frac{1}{2}} x+x^{*}\right)
$$

satisfies equation (1.1), and the estimates of Proposition 4.1 with $\ell^{*}(S)=1, x^{*}(S)=$ 0 and $S$ blows up backward at the origin in time. In particular, there exist $\varepsilon(t)$, $b(t), \lambda(t)$ and $x(t)$ such that

$$
\begin{gathered}
S(t, x)=\frac{1}{\lambda^{\frac{1}{2}}(t)}\left(Q_{b(t)}+\varepsilon\right)\left(t, \frac{x-x(t)}{\lambda(t)}\right), \\
b(t)=-t^{2}+O\left(t^{4}\right), \quad \lambda(t)=t+O\left(t^{3}\right), \quad x(t)=-\frac{1}{t}+\bar{c} t+O\left(t^{2}\right), \\
\|\varepsilon(t)\|_{L^{2}} \lesssim t, \quad \int\left(e^{-\frac{|y|}{10}}+\mathbf{1}_{y>0}(y)\right)\left(\varepsilon_{y}^{2}+\varepsilon^{2}\right)(t, y) d y \lesssim t^{6} .
\end{gathered}
$$

We now prove (4.33). Since

$$
\left\|\frac{1}{\lambda^{\frac{1}{2}}(t)} \varepsilon\left(t, \frac{-x(t)}{\lambda(t)}\right)\right\|_{L^{2}}=\|\varepsilon(t)\|_{L^{2}} \lesssim t
$$

we are reduced to estimate

$$
\begin{aligned}
& \left\|\frac{1}{\lambda^{\frac{1}{2}}(t)} Q_{b(t)}\left(\frac{.-x(t)}{\lambda(t)}\right)-\frac{1}{t^{\frac{1}{2}}} Q\left(\frac{.+\frac{1}{t}+\bar{c} t}{t}\right)\right\|_{L^{2}} \\
& =\left\|Q_{b(t)}-\frac{\lambda^{\frac{1}{2}}(t)}{t^{\frac{1}{2}}} Q\left(\frac{\lambda(t)}{t} x+\frac{1}{t}\left(\frac{1}{t}+\bar{c} t+x(t)\right)\right)\right\|_{L^{2}} \\
& \lesssim|b(t)|^{\frac{5}{8}}+\left|1-\frac{\lambda(t)}{t}\right|+\left|\frac{1}{t}\left(\frac{1}{t}+\bar{c} t+x(t)\right)\right| \lesssim t,
\end{aligned}
$$

and (4.33) is proved.

Let (see (4.36) $)$

$A(t)=\inf _{\lambda_{0}>0, x_{0} \in \mathbb{R}}\left\|S(t)-Q_{\lambda_{0}}\left(.-x_{0}\right)\right\|_{L^{2}}^{2}=\inf _{\lambda_{1}>0, x_{1} \in \mathbb{R}}\left\|Q_{b(t)}+\varepsilon(t)-Q_{\lambda_{1}}\left(.-x_{1}\right)\right\|_{L^{2}}^{2}$.

Let $\lambda_{1}(t)$ and $x_{1}(t)$ realizing the infimum in the definition of $A(t)$. (The existence, uniqueness and regularity of $\lambda_{1}(t)$ and $x_{1}(t)$ follow by standard arguments.)

Note that by extremality of $\lambda_{1}$ and $x_{1}$,

$$
\int\left(Q_{b}+\varepsilon-Q_{\lambda_{1}}\left(.-x_{1}\right)\right) \frac{\partial Q_{\lambda_{1}}}{\partial \lambda_{1}}\left(.-x_{1}\right)=0, \quad \int\left(Q_{b}+\varepsilon-Q_{\lambda_{1}}\left(.-x_{1}\right)\right) \frac{\partial Q_{\lambda_{1}}}{\partial x_{1}}\left(.-x_{1}\right)=0,
$$

and by $\|S(t)\|_{L^{2}}=\left\|Q_{b}+\varepsilon\right\|_{L^{2}}=\|Q\|_{L^{2}}$,

$$
\int\left(Q_{b}+\varepsilon\right) \frac{\partial}{\partial t}\left(Q_{b}+\varepsilon\right)=0
$$


so that

$$
\begin{aligned}
& \frac{1}{2} \frac{d}{d t} A(t) \\
& =\int\left(Q_{b}+\varepsilon-Q_{\lambda_{1}}\left(.-x_{1}\right)\right)\left(\frac{\partial}{\partial t}\left(Q_{b}+\varepsilon\right)-\lambda_{1}^{\prime} \frac{\partial Q_{\lambda_{1}}}{\partial \lambda_{1}}\left(.-x_{1}\right)+x_{1}^{\prime} \frac{\partial Q_{\lambda_{1}}}{\partial x_{1}}\left(.-x_{1}\right)\right) \\
& =-\int Q_{\lambda_{1}}\left(.-x_{1}\right) \frac{\partial}{\partial t}\left(Q_{b}+\varepsilon\right)=-\int Q_{\lambda_{1}}\left(.-x_{1}\right)\left(b_{t} \frac{\partial Q_{b}}{\partial b}+\varepsilon_{t}\right) \\
& =-b_{t} \int Q_{\lambda_{1}}\left(.-x_{1}\right) \frac{\partial Q_{b}}{\partial b}-\int \varepsilon_{t}\left(Q_{\lambda_{1}}\left(.-x_{1}\right)-Q-\left(\lambda_{1}-1\right) \Lambda Q\right) .
\end{aligned}
$$

where we have used at last $\int \varepsilon Q=\int \varepsilon \Lambda Q=0$.

To estimate this term, we now claim that from (4.39) and (4.37), (4.38),

$$
\begin{gathered}
\left|\lambda_{1}-1\right| \lesssim\left|\int\left(Q_{b}+\varepsilon-Q\right) \Lambda Q\right| \lesssim t^{2}, \\
\left|x_{1}\right| \lesssim\left|\int\left(Q_{b}+\varepsilon-Q\right) Q^{\prime}\right| \lesssim t^{3},
\end{gathered}
$$

(the extra smallness of $\left|x_{1}\right|$ is due to $\left.\left(P, Q^{\prime}\right)=0\right)$. Using $b_{t} \sim-2 t$ and the equation of $\varepsilon_{t}$ (after integration by parts, and using (4.37) and (4.38)), we obtain

$$
\frac{1}{2} \frac{d}{d t} A(t)=2 t \int P Q+O\left(t^{2}\right)
$$

\section{Uniqueness}

We prove in this section the uniqueness statement, i.e. part (ii) of Theorem 1.3. The stategy is to rerun the monotonicity machinery of Proposition 2.9 for the difference of two solutions. The reintegration of the Lyapounov functional backwards from blow up time using the sharp a priori bounds of Proposition 4.1 will yield that this difference is zero. The proof is delicate because like in [53], we only have a finite order expansion of the approximate solution and of the error. Therefore, reintegrating the difference of the modulation equations requires sharp dispersive controls on the difference of two solutions to close the estimates.

5.1. Reduction of the proof. We consider $S(t, x)=u_{1}(t, x)$ the minimal mass blow up solution constructed in Corollary 4.3, Let $u_{2}(t)$ be another minimal mass solution of (1.1) which blows up in finite time. From Proposition 4.1, $u_{2}(t)$ is defined on a maximal interval of time of the form $(-\infty, T)$ or $(T,+\infty)$ for a finite time $T$. By time translation invariance, we may assume that $u_{2}(t)$ is defined on $(0,+\infty)$ and blows backwards as $t \downarrow 0$. Let $t_{0}>0$ small such that $u_{1}$ and $u_{2}$ admit the decomposition of Lemma 2.6 on $\left(0, t_{0}\right]$ (see also Lemma 2.11)

$$
\varepsilon_{i}(s, y)=\lambda_{i}^{\frac{1}{2}}(s) u_{i}\left(t_{i}(s), \lambda_{i}(s) y+x_{i}(s)\right)-Q_{b_{i}(s)}(y),
$$

where $t_{i}(s)$ satisfies $\frac{d t_{i}}{d s}=\lambda_{i}^{3}, t_{i}(-1)=t_{0}$. Applying Proposition 4.1 to $u_{2}$, estimates (4.2) -(4.12) hold for $u_{2}(t)$, for some $\ell^{*}\left(u_{2}\right)$ and $x^{*}\left(u_{2}\right)$.

Using scaling and translation invariances (see Remark 4.2), we assume further that the limits as defined in Proposition 4.1 are equal:

$$
\ell^{*}\left(u_{2}\right)=\ell^{*}(S)=1, \quad x^{*}\left(u_{2}\right)=x^{*}(S)=0 .
$$


The uniqueness statement reduces to proving that

$$
u_{1} \equiv u_{2} \text {. }
$$

Note that for $i=1,2, \varepsilon_{i}$ satisfies on $(-\infty,-1] \times \mathbb{R}$,

$$
\left(\varepsilon_{i}\right)_{s}-\left(L \varepsilon_{i}\right)_{y}+b_{i} \Lambda \varepsilon_{i}=\Gamma_{i}\left(\Lambda Q_{b_{i}}+\Lambda \varepsilon_{i}\right)+X_{i}\left(Q_{b_{i}}+\varepsilon_{i}\right)_{y}+\Psi_{i}-\left(R_{i}\left(\varepsilon_{i}\right)\right)_{y},
$$

with

$$
\begin{gathered}
\Gamma_{i}=\frac{\left(\lambda_{i}\right)_{s}}{\lambda_{i}}+b_{i}, \quad X_{i}=\frac{\left(x_{i}\right)_{s}}{\lambda_{i}}-1 \\
\Psi_{i}=\Psi_{b_{i}}-\left(b_{i}\right)_{s}\left(\chi_{b_{i}}+\gamma y\left(\chi_{b_{i}}\right)_{y}\right) P, \quad \Psi_{b} \text { being defined in (2.13) } \\
R_{i}\left(\varepsilon_{i}\right)=5\left(Q_{b_{i}}^{4}-Q^{4}\right) \varepsilon_{i}+\left(\varepsilon_{i}+Q_{b_{i}}\right)^{5}-5 Q_{b_{i}}^{4} \varepsilon_{i}-Q_{b_{i}}^{5} .
\end{gathered}
$$

We form the difference

$$
\varepsilon(s, y)=\varepsilon_{2}(s, y)-\varepsilon_{1}(s, y)
$$

which satisfies the orthogonality conditions (2.21) and the equation:

$$
\varepsilon_{s}-(L \varepsilon)_{y}=\Gamma \Lambda Q_{b_{2}}+X\left(Q_{b_{2}}\right)_{y}+\frac{\left(\lambda_{2}\right)_{s}}{\lambda_{2}} \Lambda \varepsilon+E+F_{y}
$$

with:

$$
\begin{gathered}
\Gamma=\Gamma_{2}-\Gamma_{1}, \quad b=b_{2}-b_{1}, \quad X=X_{2}-X_{1}, \\
E=(\Gamma-b) \Lambda \varepsilon_{1}+\Gamma_{1} \Lambda\left(Q_{b_{2}}-Q_{b_{1}}\right)+\left(\Psi_{2}-\Psi_{1}\right), \\
F=X_{1}\left(Q_{b_{2}}-Q_{b_{1}}\right)+X_{2} \varepsilon+X \varepsilon_{1}-R_{2}\left(\varepsilon_{2}\right)+R_{1}\left(\varepsilon_{1}\right) .
\end{gathered}
$$

For $B$ as in Proposition 2.9, we consider $100<\bar{B}<\frac{1}{50} B$, large enough (in the next lemma, we need $\bar{B}$ large, so we take a possibly larger universal $B$ in Proposition 2.9). We define the norms:

$$
\begin{gathered}
\overline{\mathcal{N}}(s)=\int \varepsilon_{y}^{2}(s, y) \psi_{\bar{B}}(y) d y+\int \varepsilon^{2}(s, y) \varphi_{\bar{B}}(y) d y, \\
\overline{\mathcal{N}}_{\text {loc }}(s)=\int \varepsilon^{2}(s, y) \varphi_{\bar{B}}^{\prime}(y) d y .
\end{gathered}
$$

The key to the proof of uniqueness is the following Proposition which revisits Proposition 2.9 for $\varepsilon$ :

Proposition 5.1 (Bounds on the difference). For $|s|$ large, there holds the bounds: (i) Refined control of $b$ : Let

$$
J_{2}=\left(\varepsilon, \rho_{2}\right)
$$

with $\rho_{2}$ given by (2.39). Then,

$$
\left|J_{2}\right| \lesssim \overline{\mathcal{N}}^{\frac{1}{2}}, \quad\left|\frac{d}{d s}\left\{s^{2}\left(b+\frac{J_{2}}{2 s}\right)\right\}\right| \lesssim s^{2} \int \varepsilon^{2} e^{-\frac{|y|}{10}}+|s|^{\frac{1}{2}}|b|+|s|^{\frac{1}{2}} \overline{\mathcal{N}}^{\frac{1}{2}}
$$

(ii) Refined bounds: let

$$
\begin{aligned}
& \mathcal{F}(s) \\
= & \int\left[\psi_{\bar{B}}\left(\varepsilon_{y}^{2}-5 Q^{4} \varepsilon^{2}-\frac{\varepsilon^{6}}{3}\right)+\varphi_{\bar{B}} \varepsilon^{2}\right](s, y) d y+\frac{1}{\sqrt{|s|}} \int e^{\lambda_{2}(s) y} \varepsilon^{2}(s, y) d y,
\end{aligned}
$$

then:

$$
\overline{\mathcal{N}}+\frac{1}{\sqrt{|s|}} \int e^{\lambda_{2} y} \varepsilon^{2} \lesssim \mathcal{F} \lesssim \overline{\mathcal{N}}+\frac{1}{\sqrt{|s|}} \int e^{\lambda_{2} y} \varepsilon^{2} .
$$

Moreover, there exists $\mu>0$ such that, for $|s|$ large,

$$
\frac{d}{d s}\left(s^{2} \mathcal{F}\right)+\mu s^{2} \int\left(\varepsilon_{y}^{2}+\varepsilon^{2}\right) \varphi_{\bar{B}}^{\prime} \lesssim|s|^{\frac{11}{10}} b^{2} .
$$


Remark 5.2. The first term in the definition of $\mathcal{F}$ in (5.6) corresponds to a refined combination of viriel estimates and monotonicity properties which was used in 33. (see also Proposition 2.9 of the present paper). Unfortunatly, the scaling term of the equation of $\varepsilon$, i.e. the term $\frac{\left(\lambda_{2}\right)_{s}}{\lambda_{2}} \Lambda \varepsilon$, produces bad apriori lower order terms which prevent us from closing the estimates as in [33. To control these terms we have to add to the definition of $\mathcal{F}$ the second term $\frac{1}{\sqrt{|s|}} \int e^{\lambda_{2} y} \varepsilon^{2}$ which is a lower order corrective term. Note that this term is scaling invariant and thus it does not produce such bad terms.

The next two sections are devoted to the proof of Proposition 5.1

5.2. Proof of (i). We start with the control of the modulation parameters and the proof of the improved bound (5.5).

step 1 Modulation equations. We start with computing the modulation equations and claim the bounds:

$$
\begin{gathered}
|\Gamma|+|X| \lesssim\left(\int \varepsilon^{2} e^{-\frac{|y|}{10}}\right)^{\frac{1}{2}}+\frac{|b|}{|s|} \\
\left|\Gamma-\frac{\left(\varepsilon, L(\Lambda Q)^{\prime}\right)}{\|\Lambda Q\|_{L^{2}}^{2}}\right| \lesssim \int \varepsilon^{2} e^{-\frac{|y|}{10}}+\frac{1}{|s|}\left(\int \varepsilon^{2} e^{-\frac{|y|}{10}}\right)^{\frac{1}{2}}+\frac{|b|}{|s|} \\
\left|b_{s}\right| \lesssim \int \varepsilon^{2} e^{-\frac{|y|}{10}}+\frac{1}{|s|}\left(\int \varepsilon^{2} e^{-\frac{|y|}{10}}\right)^{\frac{1}{2}}+\frac{|b|}{|s|}
\end{gathered}
$$

Indeed, we compute the modulation parameters $\Gamma, X$ using (5.2) and the orthogonality conditions (2.21). We argue like for the proof of (2.29), (2.30) (see [33]) taking the scalar product of the equation of $\varepsilon$ by $\Lambda Q, y \Lambda Q$ and then by $Q$. We obtain

$$
\begin{aligned}
|\Gamma|+|X| & \lesssim\left(\int \varepsilon^{2} e^{-\frac{|y|}{10}}\right)^{\frac{1}{2}}+|b|\left(\left|b_{1}\right|+|b|+\left|\Gamma_{1}\right|+\left|X_{1}\right|+\left(\int \varepsilon_{1}^{2} e^{-\frac{|y|}{10}}\right)^{\frac{1}{2}}\right)+\left|b_{s}\right| \\
\left|b_{s}\right| & \lesssim \int \varepsilon^{2} e^{-\frac{|y|}{10}}+\frac{\left(\int \varepsilon^{2} e^{-\frac{|y|}{10}}\right)^{\frac{1}{2}}}{|s|} \\
& +|b|\left(\left|b_{1}\right|+|b|+\left|\Gamma_{1}\right|+\left|X_{1}\right|+\left(\int \varepsilon_{1}^{2} e^{-\frac{|y|}{10}}\right)^{\frac{1}{2}}\right)+\left|b_{1}\right|(|\Gamma|+|X|) .
\end{aligned}
$$

Next, using estimates (4.6)-(4.9) for $\varepsilon_{1}$, we find (5.9) and (5.11).

Note that estimate (5.9) can be improved into

$$
\begin{aligned}
\left|\Gamma-\frac{\left(\varepsilon, L(\Lambda Q)^{\prime}\right)}{\|\Lambda Q\|_{L^{2}}^{2}}\right| & \lesssim \int \varepsilon^{2} e^{-\frac{|y|}{10}}+\left(|b|+\frac{1}{|s|}\right)\left(\int \varepsilon^{2} e^{-\frac{|y|}{10}}\right)^{\frac{1}{2}}+\frac{|b|}{|s|} \\
& \lesssim \int \varepsilon^{2} e^{-\frac{|y|}{10}}+\frac{1}{|s|}\left(\int \varepsilon^{2} e^{-\frac{|y|}{10}}\right)^{\frac{1}{2}}+\frac{|b|}{|s|}
\end{aligned}
$$

which is (5.10).

step 2 Proof of (i). The estimate $\left|J_{2}\right| \lesssim \overline{\mathcal{N}}^{\frac{1}{2}}$ follows from the properties of $\rho_{2}$ :

$$
\left|\rho_{2}\right| \lesssim \mathbf{1}_{y>0}+e^{-\frac{|y|}{10}} \mathbf{1}_{y<0} .
$$


We now turn to the proof of the refined equation of $b$. We claim the bound:

$$
\left|b_{s}+4 b_{2} b+b_{2}\left(J_{2}\right)_{s}\right| \lesssim \int \varepsilon^{2} e^{-\frac{|y|}{10}}+\frac{\overline{\mathcal{N}}^{\frac{1}{2}}}{|s|^{\frac{3}{2}}}+\frac{|b|}{|s|^{\frac{3}{2}}}
$$

which follows from combining the following two estimates:

$$
\begin{gathered}
\left|b_{s}+4 b_{2} b-b_{2}\left(\varepsilon, L\left(\rho_{2}^{\prime}\right)\right)\right| \lesssim \int \varepsilon^{2} e^{-\frac{|y|}{10}}+\frac{\left(\int \varepsilon^{2} e^{-\frac{|y|}{10}}\right)^{\frac{1}{2}}}{|s|^{\frac{3}{2}}}+\frac{|b|}{|s|^{\frac{3}{2}}}, \\
\left|\left(J_{2}\right)_{s}+\left(\varepsilon, L\left(\rho_{2}^{\prime}\right)\right)\right| \lesssim \int \varepsilon^{2} e^{-\frac{|y|}{10}}+\frac{\overline{\mathcal{N}}^{\frac{1}{2}}}{|s|}+\frac{|b|}{|s|}
\end{gathered}
$$

Assume (5.13), (5.14), then from (5.14):

$$
\left|\left(J_{2}\right)_{s}\right| \lesssim \int \varepsilon^{2} e^{-\frac{|y|}{10}}+\overline{\mathcal{N}}^{\frac{1}{2}}+\frac{|b|}{|s|}
$$

and expanding $b_{2}$ in (5.12) according to (4.9) yields (5.5).

Proof of (5.13). Taking the scalar product of the equation of $\varepsilon$ by $Q$, we obtain (using $L Q^{\prime}=0$ )

$$
\begin{aligned}
0=\frac{d}{d s}(\varepsilon, Q) & =\Gamma\left(\Lambda Q_{b_{2}}, Q\right)+X\left(\left(Q_{b_{2}}\right)_{y}, Q\right)+\frac{\left(\lambda_{2}\right)_{s}}{\lambda_{2}}(\Lambda \varepsilon, Q) \\
& +(E, Q)-\left(F-\varepsilon^{5}, Q^{\prime}\right) .
\end{aligned}
$$

Using the definition of $Q_{b}$ in (2.10), we have $\left|\left(\Lambda Q_{b_{2}}, Q\right)-(\Lambda P, Q) b_{2}\right| \lesssim|s|^{-10}$, and thus using (5.10), we obtain

$$
\left|\Gamma\left(\Lambda Q_{b_{2}}, Q\right)-b_{2}(\Lambda P, Q) \frac{\left(\varepsilon, L(\Lambda Q)^{\prime}\right)}{\|\Lambda Q\|_{L^{2}}^{2}}\right| \lesssim \frac{\int \varepsilon^{2} e^{-\frac{|y|}{10}}}{|s|}+\frac{|b|}{|s|^{2}}+\frac{\left(\int \varepsilon^{2} e^{-\frac{|y|}{10}}\right)^{\frac{1}{2}}}{s^{2}} .
$$

Similarly, since $\left|\left(\left(Q_{b_{2}}\right)_{y}, Q\right)\right| \lesssim|s|^{-10}$, using (5.9),

$$
\left|X\left(\left(Q_{b_{2}}\right)_{y}, Q\right)\right| \leq \frac{\left(\int \varepsilon^{2} e^{-\frac{|y|}{10}}\right)^{\frac{1}{2}}+|b|}{|s|^{10}} .
$$

Now, we compute $(E, Q)$. By the expression of $\Psi_{b}$ (see equation $(2.17)$ in [33]), and the formula $\left(\left(10 P^{2} Q^{3}\right)^{\prime}+\Lambda P, Q\right)=\frac{1}{8}\|Q\|_{L^{1}}^{2}$, we have

$$
\left(\Psi_{b_{2}}-\Psi_{b_{1}}, Q\right)=-\frac{1}{4} b_{2} b\|Q\|_{L^{1}}^{2}+O\left(\frac{b}{s^{2}}\right) .
$$

Next, using the expression of $\Phi_{b}$ and $(P, Q)=\frac{1}{16}\|Q\|_{L^{1}}^{2}$,

$$
\left(\Phi_{b_{2}}-\Phi_{b_{1}}, Q\right)=-\frac{b_{s}}{16}\|Q\|_{L^{1}}^{2}+O\left(\frac{b}{s^{10}}\right) .
$$

Thus,

$$
\left(\Psi_{2}-\Psi_{1}, Q\right)=-\frac{1}{16}\|Q\|_{L^{1}}^{2}\left(b_{s}+4 b_{2} b\right)+O\left(\frac{b}{s^{2}}\right) .
$$

Since $\left(\Lambda \varepsilon_{1}, Q\right)=-\left(\varepsilon_{1}, \Lambda Q\right)=0$ and (using (4.8) on $\left.\varepsilon_{1}\right)$

$$
\left|\Gamma_{1}\left(\Lambda\left(Q_{b_{2}}-Q_{b_{1}}\right), Q\right)\right| \lesssim|s|^{-\frac{3}{2}}|b|,
$$


we obtain

$$
(E, Q)=-\frac{1}{16}\|Q\|_{L^{1}}^{2}\left(b_{s}+4 b_{2} b\right)+O\left(\frac{b}{|s|^{\frac{3}{2}}}\right) .
$$

Now, we compute $\left(F-\varepsilon^{5}, Q^{\prime}\right)$. First, since $\left|X_{1}\right|+\left|X_{2}\right|+\int\left|\varepsilon_{1}\right| e^{-\frac{|y|}{10}} \lesssim|s|^{-\frac{3}{2}}$, we have

$$
\left|X_{1}\left(Q_{b_{2}}-Q_{b_{1}}, Q^{\prime}\right)\right|+\left|X_{2}\left(\varepsilon, Q^{\prime}\right)\right|+\left|X\left(\varepsilon_{1}, Q^{\prime}\right)\right| \lesssim \frac{\left(\int \varepsilon^{2} e^{-\frac{|y|}{10}}\right)^{\frac{1}{2}}}{|s|^{\frac{3}{2}}}+\frac{|b|}{|s|^{\frac{3}{2}}} .
$$

Second, we estimate $\left(R_{2}\left(\varepsilon_{2}\right)-R_{1}\left(\varepsilon_{1}\right), Q^{\prime}\right)$. From the expression of $R_{i}\left(\varepsilon_{i}\right)$, we observe that

$$
\begin{aligned}
& \left|R_{2}\left(\varepsilon_{2}\right)-R_{1}\left(\varepsilon_{1}\right)-20 b_{2} P Q^{3} \varepsilon\right| \\
& \leq\left|R_{2}\left(\varepsilon_{2}\right)-R_{2}\left(\varepsilon_{1}\right)-20 b_{2} P Q^{3} \varepsilon\right|+\left|R_{2}\left(\varepsilon_{1}\right)-R_{1}\left(\varepsilon_{1}\right)\right| \\
& \lesssim|\varepsilon|\left(|s|^{-2}+\left|\varepsilon_{1}\right|+\left|\varepsilon_{2}\right|\right)+|b|\left|\varepsilon_{1}\right|,
\end{aligned}
$$

and so by (4.7),

$$
\left|\left(R_{2}\left(\varepsilon_{2}\right)-R_{1}\left(\varepsilon_{1}\right), Q^{\prime}\right)-20 b_{2}\left(\varepsilon, P Q^{3} Q^{\prime}\right)\right| \lesssim \frac{\left(\int \varepsilon^{2} e^{-\frac{|y|}{10}}\right)^{\frac{1}{2}}}{|s|^{\frac{3}{2}}}+\frac{|b|}{|s|^{\frac{3}{2}}} .
$$

We have thus obtained for this term:

$$
\left|\left(\left(F-\varepsilon^{5}\right)_{y}, Q\right)+20 b_{2}\left(\varepsilon, P Q^{3} Q^{\prime}\right)\right| \lesssim \frac{\left(\int \varepsilon^{2} e^{-\frac{|y|}{10}}\right)^{\frac{1}{2}}}{|s|^{\frac{3}{2}}}+\frac{|b|}{|s|^{\frac{3}{2}}} .
$$

Inserting the above computations into (5.15), we obtain:

$$
\begin{aligned}
& \left|b_{s}+4 b_{2} b-\frac{16}{\|Q\|_{L^{1}}^{2}} b_{2}\left[\frac{(\Lambda P, Q)}{\|\Lambda Q\|_{L^{2}}^{2}}\left(\varepsilon, L(\Lambda Q)^{\prime}\right)+20\left(\varepsilon, P Q^{3} Q^{\prime}\right)\right]\right| \\
& \lesssim \int \varepsilon^{2} e^{-\frac{|y|}{10}}+\frac{\left(\int \varepsilon^{2} e^{-\frac{|y|}{10}}\right)^{\frac{1}{2}}}{|s|^{\frac{3}{2}}}+\frac{|b|}{|s|^{\frac{3}{2}}}
\end{aligned}
$$

Using the following computation 8 from [33], proof of Lemma 2.7:

$$
\left(\varepsilon, L\left(\rho_{2}^{\prime}\right)\right)=\frac{16}{\|Q\|_{L^{1}}^{2}}\left[\frac{(\Lambda P, Q)}{\|\Lambda Q\|_{L^{2}}^{2}}\left(\varepsilon, L(\Lambda Q)^{\prime}\right)+20\left(\varepsilon, P Q^{3} Q^{\prime}\right)\right],
$$

we obtain (5.13).

Proof of (5.14): To complete the proof of (5.12), we take the scalar product of the equation of $\varepsilon$ by $\rho_{2}$. We obtain first:

$$
\begin{aligned}
\frac{d}{d s} J_{2}+\frac{\left(\lambda_{2}\right)_{s}}{\lambda_{2}}\left(\varepsilon, \Lambda \rho_{2}\right) & =-\left(\varepsilon,\left(L \rho_{2}\right)^{\prime}\right)+\Gamma\left(\Lambda Q_{b_{2}}, \rho_{2}\right)+X\left(\left(Q_{b_{2}}\right)_{y}, \rho_{2}\right) \\
& +\left(E, \rho_{2}\right)+\left(-F+\varepsilon^{5}, \rho_{2}^{\prime}\right) .
\end{aligned}
$$

Note that

$$
\left|\frac{\left(\lambda_{2}\right)_{s}}{\lambda_{2}}\left(\varepsilon, \Lambda \rho_{2}\right)\right| \leq\left|\frac{\left(\lambda_{2}\right)_{s}}{\lambda_{2}}\right|\left(\left|J_{2}\right|+\left|\left(\varepsilon, y \rho_{2}^{\prime}\right)\right|\right) \lesssim \frac{\overline{\mathcal{N}}^{\frac{1}{2}}}{|s|} .
$$

\footnotetext{
${ }^{8}$ which of course motivates the definition of $\rho_{2}$ in (2.39).
} 
Using the orthogonality $\left(\Lambda Q, \rho_{2}\right)=0$ (see [33]), and (5.9), we have

$$
\left|\Gamma\left(\Lambda Q_{b_{2}}, \rho_{2}\right)\right|=\left|\Gamma b_{2}\left(\Lambda P \chi_{b_{2}}, \rho_{2}\right)\right| \lesssim\left[\left(\int \varepsilon^{2} e^{-\frac{|y|}{10}}\right)^{\frac{1}{2}}+\frac{|b|}{|s|}\right] \frac{1}{|s|}
$$

and similarly using $\left(Q, \rho_{2}^{\prime}\right)=0$ :

$\left|X\left(\left(Q_{b_{2}}\right)_{y}, \rho_{2}\right)\right|+\left|\left(E, \rho_{2}\right)\right|+\left|\left(-F+\varepsilon^{5}, \rho_{2}^{\prime}\right)\right| \lesssim \frac{\left(\int \varepsilon^{2} e^{-\frac{|y|}{10}}\right)^{\frac{1}{2}}+|b|+\overline{\mathcal{N}}^{\frac{1}{2}}}{|s|}+\int \varepsilon^{2} e^{-\frac{|y|}{10}}$,

and (5.14) is proved.

5.3. Proof of (ii). The functional $\mathcal{F}$ in $(5.6)$ is defined similarly as in Proposition 2.9 for a parameter $\bar{B}$ large enough but smaller than $B / 10$ where $B$ is used in Proposition 2.9.

step 1 Coercivity of $\mathcal{F}$. The upper and lower bounds (5.7) on $\mathcal{F}$ follow from the coercivity of the linearized energy $\int \varepsilon_{y}^{2}+\varepsilon^{2}-5 Q^{4} \varepsilon^{2}$ under the orthogonality conditions (2.21) together with standard localization arguments. We refer to the proof of Proposition 3.1 (iii) in [33] for example for more details.

step 2 Proof of (5.8). We now turn to the proof of the monotonicity (5.8). We decompose $\mathcal{F}=\overline{\mathcal{F}_{1}}+\frac{1}{\sqrt{|s|}} \overline{\mathcal{F}_{2}}$ with

$$
\overline{\mathcal{F}_{1}}=\int\left[\psi_{\bar{B}}\left(\varepsilon_{y}^{2}-5 Q^{4} \varepsilon^{2}\right)+\varphi_{\bar{B}} \varepsilon^{2}\right], \quad \overline{\mathcal{F}_{2}}=\int e^{\lambda_{2} y} \varepsilon^{2}
$$

and claim the monotonicity formulas for $|s|$ large enough:

$$
\begin{gathered}
\frac{d \overline{\mathcal{F}_{1}}}{d s}+\mu_{1} \int\left(\varepsilon_{y}^{2}+\varepsilon^{2}\right) \varphi_{\bar{B}}^{\prime} \lesssim \frac{b^{2}}{|s|}+\frac{1}{|s|} \int_{y<0}|y| e^{-\frac{|y|}{\bar{B}}} \varepsilon^{2}, \\
\frac{d \overline{\mathcal{F}_{2}}}{d s}+\frac{\mu_{2}}{\sqrt{|s|}} \int e^{\lambda_{2} y}\left(\varepsilon_{y}^{2}+\varepsilon^{2}\right) \lesssim \int \varepsilon^{2} e^{-\frac{|y|}{10}}+\frac{|b|^{2}}{|s|^{\frac{2}{5}}} .
\end{gathered}
$$

Assume (5.17), (5.18), then for $|s|$ large:

$$
\begin{aligned}
& \frac{d}{d s}\left(s^{2} \overline{\mathcal{F}_{1}}+|s|^{\frac{3}{2}} \overline{\mathcal{F}_{2}}\right)=2 s \overline{\mathcal{F}_{1}}-\frac{3}{2}|s|^{\frac{1}{2}} \overline{\mathcal{F}_{2}}+s^{2} \frac{d}{d s} \overline{\mathcal{F}_{1}}+|s|^{\frac{3}{2}} \frac{d}{d s} \overline{\mathcal{F}_{2}} \\
& \leq-\mu_{1} s^{2} \int\left(\varepsilon_{y}^{2}+\varepsilon^{2}\right) \varphi^{\prime} \bar{B}-\mu_{2}|s| \int e^{\lambda_{2} y} \varepsilon^{2}+C|s|^{\frac{11}{10}} b^{2}+C|s| \int_{y<0}|y| e^{-\frac{|y|}{\bar{B}}} \varepsilon^{2} \\
& +C|s|^{\frac{3}{2}} \int \varepsilon^{2} e^{-\frac{|y|}{10}} \\
& \leq-\frac{\mu_{1}}{2} s^{2} \int\left(\varepsilon_{y}^{2}+\varepsilon^{2}\right) \varphi_{\bar{B}}^{\prime}-\mu_{2}|s| \int e^{\lambda_{2} y} \varepsilon^{2}+C|s|^{\frac{11}{10}} b^{2}+C_{1}|s| \int_{y<0}|y| e^{-\frac{|y|}{B}} \varepsilon^{2} .
\end{aligned}
$$

Then, for $0<\kappa<\mu_{1} /\left(4 \bar{B} C_{1}\right)$ and $|s|$ large enough (depending on $\bar{B}$ ),

$$
\begin{aligned}
C_{1}|s| \int_{y<0}|y| e^{-\frac{|y|}{\bar{B}}} \varepsilon^{2} & =C_{1}|s| \int_{\kappa s<y<0}|y| e^{-\frac{|y|}{\bar{B}}} \varepsilon^{2}+C_{1}|s| \int_{y<\kappa s}|y| e^{-\frac{|y|}{\bar{B}}} \varepsilon^{2} \\
& \leq C_{1} \bar{B} \kappa s^{2} \overline{\mathcal{N}}_{\text {loc }}+C_{1}|s| \sup _{y<\kappa s}\left(|y| e^{-\frac{|y|}{2 \bar{B}}}\right) \int e^{-\frac{|y|}{2 \bar{B}} \varepsilon^{2}} \\
& \leq \frac{\mu_{1}}{4} s^{2} \overline{\mathcal{N}}_{\text {loc }}+\frac{\mu_{2}}{2}|s| \int e^{\lambda_{2} y} \varepsilon^{2},
\end{aligned}
$$


and thus

$$
\frac{d}{d s}\left(s^{2} \overline{\mathcal{F}_{1}}+|s|^{\frac{3}{2}} \overline{\mathcal{F}_{2}}\right)+\frac{\mu_{1}}{4} s^{2} \int\left(\varepsilon_{y}^{2}+\varepsilon^{2}\right) \varphi_{\bar{B}}^{\prime}+\frac{\mu_{2}}{2}|s| \int e^{\lambda_{2} y} \varepsilon^{2} \lesssim|s|^{\frac{11}{10}} b^{2}
$$

which implies (5.8).

step 3 Proof of (5.17). We compute the time derivative of $\overline{\mathcal{F}_{1}}$ using (5.2):

$$
\begin{aligned}
\frac{1}{2} \frac{d \overline{\mathcal{F}_{1}}}{d s}= & \int \partial_{s} \varepsilon\left[-\psi_{\bar{B}}^{\prime} \varepsilon_{y}+\psi_{\bar{B}}(L \varepsilon)+\left(\varphi_{\bar{B}}-\psi_{\bar{B}}\right) \varepsilon\right] \\
= & \int\left[(L \varepsilon)_{y}+\Gamma \Lambda Q_{b_{2}}+X\left(Q_{b_{2}}\right)_{y}+\frac{\left(\lambda_{2}\right)_{s}}{\lambda_{2}} \Lambda \varepsilon+E+F_{y}\right] \\
& \times\left[-\psi \bar{B}_{\bar{B}}^{\prime} \varepsilon_{y}+\psi_{\bar{B}}(L \varepsilon)+\left(\varphi_{\bar{B}}-\psi_{\bar{B}}\right) \varepsilon\right] .
\end{aligned}
$$

We now estimate all these terms similarly as in the proof of Proposition 3.1 (i) in [33.

- First, we claim that for $\bar{B}$ large enough, for some $\mu_{1}>0$,

$$
\int(L \varepsilon)_{y}\left[-\psi_{\bar{B}}^{\prime} \varepsilon_{y}+\psi_{\bar{B}}(L \varepsilon)+\left(\varphi_{\bar{B}}-\psi_{\bar{B}}\right) \varepsilon\right] \leq-\mu_{1} \int \varphi_{\bar{B}}^{\prime}\left(\varepsilon_{y}^{2}+\varepsilon^{2}\right) .
$$

The proof is mainly based on local virial estimates for $\varepsilon$ and explicit computations similar to the ones for the term $f_{1,1}^{(i)}$ of the proof of Proposition 3.1 in [33]. Here, computations are similar and easier than in [33. We sketch these computations and estimates for the sake of completeness.

By explicit computations (mainly integrations by parts, see [33] for more details), one gets

$$
\begin{aligned}
& 2 \int(L \varepsilon)_{y}\left[-\psi_{\bar{B}}^{\prime} \varepsilon_{y}+\psi_{\bar{B}}(L \varepsilon)+\left(\varphi_{\bar{B}}-\psi_{\bar{B}}\right) \varepsilon\right] \\
& =-\int\left[3 \psi \frac{\prime}{B} \varepsilon_{y y}^{2}+\left(3 \varphi_{\bar{B}}^{\prime}+\psi \frac{\prime}{B}-\psi \frac{\prime \prime \prime}{B}\right) \varepsilon_{y}^{2}+\left(\varphi_{\bar{B}}^{\prime}-\varphi_{\frac{\prime \prime}{B}}^{\prime \prime} \varepsilon^{2}\right]\right. \\
& +\int 5 Q^{4} \varepsilon^{2}\left(\varphi_{\bar{B}}^{\prime}-\psi_{\bar{B}}^{\prime}\right)+\int 20 Q^{3} Q^{\prime} \varepsilon^{2}\left(\psi_{\bar{B}}-\varphi_{\bar{B}}\right) \\
& +10 \int \psi \psi_{\bar{B}}^{\prime} \varepsilon_{y}\left\{4 Q^{\prime} Q^{3} \varepsilon+Q^{4} \varepsilon_{y}\right\} \\
& -\int \psi \frac{1}{B}\left\{\left(5 Q^{4} \varepsilon\right)^{2}-10 Q^{4} \varepsilon\left(-\varepsilon_{y y}+\varepsilon\right)\right\} \\
& =I^{<}+I^{\sim}+I^{>}
\end{aligned}
$$

where $I^{<, \sim,>}$ respectively corresponds to integration on $y<-\frac{\bar{B}}{2},|y| \leq \frac{\bar{B}}{2}, y>\frac{\bar{B}}{2}$.

In the region $y>\bar{B} / 2$, we have $\psi_{\bar{B}}^{\prime}(y)=0$, and thus,

$$
\begin{aligned}
I^{>} & =-\int_{y>\bar{B} / 2}\left[3 \varphi_{\bar{B}}^{\prime} \varepsilon_{y}^{2}+\left(\varphi_{\bar{B}}^{\prime}-\varphi \frac{\prime \prime \prime}{B}\right) \varepsilon^{2}\right]+\int_{y>\bar{B} / 2} 5 Q^{4} \varepsilon^{2} \varphi_{\bar{B}}^{\prime} \\
& +\int_{y>\bar{B} / 2} 20 Q^{3} Q^{\prime} \varepsilon^{2}(1-\varphi \bar{B}) .
\end{aligned}
$$

Using $\varphi_{\bar{B}}^{\prime \prime \prime} \lesssim \frac{1}{\bar{B}^{2}} \varphi_{\bar{B}}^{\prime}$ and the exponential decay of $Q$, we obtain for $\bar{B}$ large enough,

$$
I^{>} \leq-\int_{y>\bar{B} / 2} \varphi_{\bar{B}}^{\prime}\left(\varepsilon_{y}^{2}+\varepsilon^{2}\right)+\frac{C}{\bar{B}^{2}} \int_{y>\bar{B} / 2} \varphi \frac{\bar{B}^{2}}{\varepsilon^{2}} \leq-\frac{1}{2} \int_{y>\bar{B} / 2} \varphi_{\bar{B}}^{\prime}\left(\varepsilon_{y}^{2}+\varepsilon^{2}\right) .
$$


In the region $|y|<\bar{B} / 2$, we have $\varphi_{\bar{B}}(y)=1+y / \bar{B}, \psi_{\bar{B}}(y)=1$ and $\psi_{\bar{B}}^{\prime \prime \prime \prime}=\psi \frac{\prime}{B}=0$. Thus,

$$
I^{\sim}=-\frac{1}{\bar{B}} \int_{|y|<\frac{\bar{B}}{2}}\left[3 \varepsilon_{y}^{2}+\varepsilon^{2}-5 Q^{4} \varepsilon^{2}+20 y Q^{3} Q^{\prime} \varepsilon^{2}\right]+\frac{1}{\bar{B}} \frac{5}{3} \int_{|y|<\frac{\bar{B}}{2}} \varepsilon^{6} .
$$

From Lemma 3.4 in [33] (local virial estimate), for some $\mu>0$ and for $\bar{B}$ large,

$$
\int_{|y|<\frac{\bar{B}}{2}}\left[3 \varepsilon_{y}^{2}+\varepsilon^{2}+15 Q^{4} \varepsilon^{2}-20 y Q^{3} Q^{\prime} \varepsilon^{2}\right] \geq \mu \int_{|y|<\frac{\bar{B}}{2}}\left(\varepsilon_{y}^{2}+\varepsilon^{2}\right)-\frac{1}{\bar{B}} \int \varepsilon^{2} e^{-\frac{|y|}{2}},
$$

and by $\|\varepsilon\|_{L^{\infty}}^{4} \lesssim|s|^{-2}, \int_{|y|<\frac{\bar{B}}{2}} \varepsilon^{6} \lesssim|s|^{-2} \int_{|y|<\frac{\bar{B}}{2}} \varepsilon^{2}$, so that for $\bar{B}$ large and $|s|$ large,

$$
I^{\sim} \leq-\frac{\mu}{2} \frac{1}{\bar{B}} \int_{|y|<\frac{\bar{B}}{2}}\left(\varepsilon_{y}^{2}+\varepsilon^{2}\right)+\frac{1}{\bar{B}^{2}} \int \varepsilon^{2} e^{-\frac{|y|}{2}} .
$$

In the region $y<-\bar{B} / 2$, we use $\psi_{\bar{B}}^{\prime \prime \prime} \lesssim \frac{1}{\bar{B}^{2}} \psi_{\bar{B}}^{\prime}, \psi_{\bar{B}}^{\prime} \lesssim \varphi_{\bar{B}}^{\prime}, \varphi_{\bar{B}}^{\prime \prime \prime} \lesssim \frac{1}{\bar{B}^{2}} \varphi_{\bar{B}}^{\prime}$, the exponential decay of $Q$ to obtain as before, for $\bar{B}$ large enough,

$$
I^{<} \leq-\frac{1}{2} \int_{y<-\bar{B} / 2} \varphi \frac{\prime}{B}\left(\varepsilon_{y}^{2}+\varepsilon^{2}\right) .
$$

Gathering the estimates for $I^{>}, I^{\sim}$ and $I^{<}$, we get (5.19).

- Next, arguing as for estimating $f_{1,2}^{(i)}$ and $f_{1,3}^{(i)}$ in the proof of Proposition 3.1 of [33, we find

$$
\begin{gathered}
\left|\Gamma \int \Lambda Q_{b_{2}}\left[-\psi_{\bar{B}}^{\prime} \varepsilon_{y}+\psi_{\bar{B}}(L \varepsilon)+\left(\varphi_{\bar{B}}-\psi_{\bar{B}}\right) \varepsilon\right]\right| \leq \frac{\mu_{1}}{100} \overline{\mathcal{N}}_{\text {loc }}+C \frac{b^{2}}{s^{2}} \\
\left|X \int\left(Q_{b_{2}}\right)_{y}\left[-\psi_{\bar{B}}^{\prime} \varepsilon_{y}+\psi_{\bar{B}}(L \varepsilon)+\left(\varphi_{\bar{B}}-\psi_{\bar{B}}\right) \varepsilon\right]\right| \leq \frac{\mu_{1}}{100} \overline{\mathcal{N}}_{\text {loc }}+C \frac{b^{2}}{s^{2}} .
\end{gathered}
$$

Indeed, using the following algebraic facts

$$
(\Lambda Q, L \varepsilon)=-2(Q, \varepsilon)=0, \quad(\varepsilon, y \Lambda Q)=(\varepsilon, \Lambda Q)=\left(\varepsilon, y Q^{\prime}\right)=0, \quad L Q^{\prime}=0,
$$

the exponential decay of $Q,\left|b_{2}\right| \leq \frac{1}{\sqrt{|s|}}$ and integrating by parts to remove all derivative from $\varepsilon$, we obtain

$$
\begin{gathered}
\left|\int \Lambda Q_{b_{2}}\left[-\psi_{\bar{B}}^{\prime} \varepsilon_{y}+\psi_{\bar{B}}(L \varepsilon)+\left(\varphi_{\bar{B}}-\psi_{\bar{B}}\right) \varepsilon\right]\right| \lesssim\left(\frac{1}{\bar{B}}+\frac{\sqrt{\bar{B}}}{\sqrt{|s|}}\right) \overline{\mathcal{N}}_{\mathrm{loc}}^{\frac{1}{2}}, \\
\left|\int\left(Q_{b_{2}}\right)_{y}\left[-\psi_{\bar{B}}^{\prime} \varepsilon_{y}+\psi_{\bar{B}}(L \varepsilon)+\left(\varphi_{\bar{B}}-\psi_{\bar{B}}\right) \varepsilon\right]\right| \lesssim\left(\frac{1}{\bar{B}}+\frac{\sqrt{\bar{B}}}{\sqrt{|s|}}\right) \overline{\mathcal{N}}_{\mathrm{loc}}^{\frac{1}{2}} .
\end{gathered}
$$

Thus, using (5.9), (5.20) and (5.21) follow for $\bar{B}$ large and $|s|$ large.

At this point, $\bar{B}$ is fixed and thus $B$ in Proposition 2.9 is also fixed. $B$ and $\bar{B}$ are universal constants.

- The next term is similar to $f_{3}^{(i, j)}$ in [33]. We have using the properties of $\psi_{\bar{B}}$ and $\varphi_{\bar{B}}$ and (2.29),

$$
\begin{aligned}
& \frac{\left(\lambda_{2}\right)_{s}}{\lambda_{2}} \int \Lambda \varepsilon\left[-\left(\psi_{\bar{B}} \varepsilon_{y}\right)_{y}+\varphi_{\bar{B}} \varepsilon-\psi_{\bar{B}}\left(5 Q^{4} \varepsilon+\varepsilon^{5}\right)\right] \\
& =-\frac{1}{2} \frac{\left(\lambda_{2}\right)_{s}}{\lambda_{2}} \int y \varphi_{\bar{B}}^{\prime} \varepsilon^{2}+O\left(\frac{1}{|s|} \int\left(\varepsilon_{y}^{2}+\varepsilon^{2}\right) \varphi_{\bar{B}}^{\prime}\right) .
\end{aligned}
$$


From (4.5), (4.8) and (4.9), we have for $s$ large

and thus

$$
\frac{1}{s} \leq-\frac{\left(\lambda_{2}\right)_{s}}{\lambda_{2}} \leq \frac{1}{4 s}<0
$$

$$
-\frac{1}{2} \frac{\left(\lambda_{2}\right)_{s}}{\lambda_{2}} \int y \varphi_{\frac{\prime}{B}}^{\prime} \varepsilon^{2} \lesssim \frac{1}{|s|} \int_{y<0}|y| e^{-\frac{|y|}{B}} \varepsilon^{2} .
$$

Eventually, we have proved for $s$ large:

$$
\begin{aligned}
& \frac{\left(\lambda_{2}\right)_{s}}{\lambda_{2}} \int \Lambda \varepsilon\left[-\left(\psi_{\bar{B}} \varepsilon_{y}\right)_{y}+\varphi_{\bar{B}} \varepsilon-\psi_{\bar{B}}\left(5 Q^{4} \varepsilon+\varepsilon^{5}\right)\right] \\
& \leq \frac{\mu_{1}}{100} \int\left(\varepsilon_{y}^{2}+\varepsilon^{2}\right) \varphi_{\bar{B}}^{\prime}+\frac{C}{|s|} \int_{y<0}|y| e^{-\frac{|y|}{B}} \varepsilon^{2} .
\end{aligned}
$$

We now estimate terms coming from $E$ and $F$. For this, we will need higher order Sobolev estimates on $\varepsilon_{1}$ and $\varepsilon_{2}$ coming from Proposition 4.1. Since $\bar{B}<\frac{B}{50}$, from (4.12) and (4.13), we have, for all $\frac{9}{50} \frac{1}{\bar{B}}<\omega<\frac{1}{10}$, for $i=1,2$,

$$
\begin{gathered}
\sum_{k=0}^{3} \int\left(\partial_{y}^{k} \varepsilon_{i}\right)^{2}(s, y) e^{\omega y} d y+\int_{-\infty}^{s} \sum_{k=0}^{4} \int\left(\partial_{y}^{k} \varepsilon_{i}\right)^{2}\left(s^{\prime}, y\right) e^{\omega y} d y d s^{\prime} \lesssim \frac{1}{|s|}, \\
\left\|\left(\left(\varepsilon_{i}\right)_{y y}^{2}+\left(\varepsilon_{i}\right)_{y}^{2}\right)(s) e^{\omega y}\right\|_{L^{\infty}} \lesssim \frac{1}{|s|} .
\end{gathered}
$$

- Estimate for $E$. In view of the expression of $E$ in (5.3), the first term to estimate is

$$
\begin{aligned}
& \left|(\Gamma-b) \int \Lambda \varepsilon_{1}\left[-\psi_{\bar{B}}^{\prime} \varepsilon_{y}+\psi_{\bar{B}}(L \varepsilon)+\left(\varphi_{\bar{B}}-\psi_{\bar{B}}\right) \varepsilon\right]\right| \\
& \lesssim\left(|b|+\left(\int \varepsilon^{2} e^{-\frac{|y|}{10}}\right)^{\frac{1}{2}}\right)\left(\int\left(\varepsilon_{y}^{2}+\varepsilon^{2}\right) \varphi_{\bar{B}}^{\prime}\right)^{\frac{1}{2}}\left(\int\left(\left(\varepsilon_{1}\right)_{y y}^{2}+\left(\varepsilon_{1}\right)_{y}^{2}+\varepsilon_{1}^{2}\right)\left(1+|y|^{3}\right) \varphi_{\bar{B}}\right)^{\frac{1}{2}} \\
& \leq \frac{\mu_{1}}{100} \int\left(\varepsilon_{y}^{2}+\varepsilon^{2}\right) \varphi_{\bar{B}}^{\prime}+C b^{2} \int\left(\left(\varepsilon_{1}\right)_{y y}^{2}+\left(\varepsilon_{1}\right)_{y}^{2}+\varepsilon_{1}^{2}\right) e^{\frac{2}{B} y} d y \\
& \leq \frac{\mu_{1}}{100} \int\left(\varepsilon_{y}^{2}+\varepsilon^{2}\right) \varphi_{\frac{1}{B}}^{\prime}+C \frac{b^{2}}{|s|}
\end{aligned}
$$

using (5.9), integration by parts, and then (5.22). For the next term, we need to estimate $\Lambda\left(Q_{b_{2}}-Q_{b_{1}}\right)$. From Lemma [2.5, we have

$$
Q_{b_{2}}-Q_{b_{1}}=b P \chi_{b_{2}}+b_{1} P\left(\chi_{b_{2}}-\chi_{b_{1}}\right)
$$

and using (4.9):

$$
\left|\chi_{b_{2}}-\chi_{b_{1}}\right|=\left|\int_{b_{1}}^{b_{2}} \frac{\partial \chi_{b}}{\partial b} d b\right| \lesssim \sup \left|y \chi^{\prime}\right| \frac{\left|b_{1}-b_{2}\right|}{\left|b_{1}\right|} .
$$

Thus, $\left|Q_{b_{2}}-Q_{b_{1}}\right| \lesssim|b||P|$. Arguing similarly, we obtain:

$$
\left|\left(Q_{b_{2}}-Q_{b_{1}}\right)_{y}\right|+\left|\left(\Lambda Q_{b_{2}}-\Lambda Q_{b_{1}}\right)_{y}\right|+\left|\Lambda\left(Q_{b_{2}}-Q_{b_{1}}\right)\right| \lesssim|b|\left(\mathbf{1}_{y<0}+e^{-\frac{|y|}{10}}\right) .
$$

Using (5.24) and estimates for $\Gamma_{1}$ from Proposition 4.1 .

$$
\begin{aligned}
& \left|\int \Gamma_{1} \Lambda\left(Q_{b_{2}}-Q_{b_{1}}\right)\left[-\psi_{\bar{B}}^{\prime} \varepsilon_{y}+\psi_{\bar{B}}\left(L \varepsilon-\varepsilon^{5}\right)+\left(\varphi_{\bar{B}}-\psi_{\bar{B}}\right) \varepsilon\right]\right| \\
& \lesssim \frac{|b|}{|s|^{\frac{3}{2}}} \overline{\mathcal{N}}_{\text {loc }}^{\frac{1}{2}} \leq \frac{\mu_{1}}{100} \overline{\mathcal{N}}_{\text {loc }}+C \frac{b^{2}}{s^{2}} .
\end{aligned}
$$


Next, from the definition of $\Psi_{b_{i}}$ and $\Phi_{b_{i}}$ (see Lemma 2.5 and equation (2.17) in [33), (5.11), (2.30), (4.7): we have

$$
\begin{aligned}
\left|\Psi_{b_{2}}-\Psi_{b_{1}}\right| & \lesssim \frac{|b|}{\sqrt{|s|}}\left(\mathbf{1}_{y<0}+e^{-\frac{|y|}{10}}\right) \\
\left|\Phi_{b_{2}}-\Phi_{b_{1}}\right| & \lesssim\left(\left|b_{s}\right|+\frac{\left|\left(b_{1}\right)_{s}\right||b|}{\left|b_{1}\right|}\right)\left(\mathbf{1}_{y<0}+e^{-\frac{|y|}{10}}\right) \\
& \lesssim\left(\int \varepsilon^{2} e^{-\frac{|y|}{10}}+\frac{\left(\int \varepsilon^{2} e^{-\frac{|y|}{10}}\right)^{\frac{1}{2}}}{|s|}+\frac{|b|}{|s|}\right)\left(\mathbf{1}_{y<0}+e^{-\frac{|y|}{10}}\right)
\end{aligned}
$$

and similar estimates for the derivatives of these terms. In particular, we obtain

$$
\begin{aligned}
& \left|\Psi_{2}-\Psi_{1}\right|+\left|\left(\Psi_{2}-\Psi_{1}\right)_{y}\right|+\left|\left(\Psi_{2}-\Psi_{1}\right)_{y y}\right| \\
& \lesssim\left(\int \varepsilon^{2} e^{-\frac{|y|}{10}}+\frac{\left(\int \varepsilon^{2} e^{-\frac{|y|}{10}}\right)^{\frac{1}{2}}}{|s|}+\frac{|b|}{\sqrt{|s|}}\right)\left(\mathbf{1}_{y<0}+e^{-\frac{|y|}{10}}\right) \\
& \lesssim\left(\overline{\mathcal{N}}_{\text {loc }}+\frac{\overline{\mathcal{N}}_{\text {loc }}^{\frac{1}{2}}}{|s|}+\frac{|b|}{\sqrt{|s|}}\right)\left(\mathbf{1}_{y<0}+e^{-\frac{|y|}{10}}\right)
\end{aligned}
$$

Thus,

$$
\begin{aligned}
& \left|\int\left(\Psi_{2}-\Psi_{1}\right)\left[-\psi_{\bar{B}}^{\prime} \varepsilon_{y}+\psi_{\bar{B}}(L \varepsilon)+\left(\varphi_{\bar{B}}-\psi_{\bar{B}}\right) \varepsilon\right]\right| \\
& \lesssim\left(\overline{\mathcal{N}}_{\text {loc }}+\frac{\overline{\mathcal{N}}_{\text {loc }}^{\frac{1}{2}}}{|s|}+\frac{|b|}{\sqrt{|s|}}\right) \overline{\mathcal{N}}_{\text {loc }}^{\frac{1}{2}} \leq \frac{\mu_{1}}{100} \overline{\mathcal{N}}_{\text {loc }}+C \frac{b^{2}}{|s|} .
\end{aligned}
$$

In conclusion for $E$, we have obtained

$$
\left|\int E\left[-\psi_{\bar{B}}^{\prime} \varepsilon_{y}+\psi_{\bar{B}}(L \varepsilon)+\left(\varphi_{\bar{B}}-\psi_{\bar{B}}\right) \varepsilon\right]\right| \leq \frac{3 \mu_{1}}{100} \int\left(\varepsilon_{y}^{2}+\varepsilon^{2}\right) \varphi_{\bar{B}}^{\prime}+C \frac{b^{2}}{|s|} .
$$

- Estimate for F. Similarly, we easily get the following three estimates

$$
\begin{aligned}
& \left|X_{1} \int\left(Q_{b_{2}}-Q_{b_{1}}\right)_{y}\left[-\psi_{\bar{B}}^{\prime} \varepsilon_{y}+\psi_{\bar{B}}(L \varepsilon)+\left(\varphi_{\bar{B}}-\psi_{\bar{B}}\right) \varepsilon\right]\right| \\
& \lesssim \frac{|b|}{|s|^{\frac{3}{2}}} \overline{\mathcal{N}}_{\text {loc }}^{\frac{1}{2}} \lesssim \frac{\mu_{1}}{100} \overline{\mathcal{N}}_{\text {loc }}+C \frac{b^{2}}{s^{2}}, \\
& \left|X_{2} \int \varepsilon_{y}\left[-\psi_{\bar{B}}^{\prime} \varepsilon_{y}+\psi_{\bar{B}}(L \varepsilon)+\left(\varphi_{\bar{B}}-\psi_{\bar{B}}\right) \varepsilon\right]\right| \\
& \lesssim \frac{1}{|s|^{\frac{3}{2}}} \int\left(\varepsilon_{y}^{2}+\varepsilon^{2}\right) \varphi_{\bar{B}}^{\prime} \leq \frac{\mu_{1}}{100} \int\left(\varepsilon_{y}^{2}+\varepsilon^{2}\right) \varphi_{\bar{B}}^{\prime},
\end{aligned}
$$




$$
\begin{aligned}
& \left|X \int\left(\varepsilon_{1}\right)_{y}\left[-\psi_{\bar{B}}^{\prime} \varepsilon_{y}+\psi_{\bar{B}}(L \varepsilon)+\left(\varphi_{\bar{B}}-\psi_{\bar{B}}\right) \varepsilon\right]\right| \\
& \lesssim\left(\frac{|b|}{|s|}+\left(\int \varepsilon^{2} e^{-\frac{|y|}{10}}\right)^{\frac{1}{2}}\right)\left(\int\left(\varepsilon_{y}^{2}+\varepsilon^{2}\right) \varphi_{\bar{B}}^{\prime}\right)^{\frac{1}{2}}\left(\int\left(\left(\varepsilon_{1}\right)_{y y}^{2}+\left(\varepsilon_{1}\right)_{y}^{2}+\varepsilon_{1}^{2}\right) e^{\frac{2}{B} y} d y\right)^{\frac{1}{2}} \\
& \leq \frac{\mu_{1}}{100} \int\left(\varepsilon_{y}^{2}+\varepsilon^{2}\right) \varphi_{\bar{B}}^{\prime}+C \frac{b^{2}}{|s|}
\end{aligned}
$$

The remaining nonlinear term for $F$ is estimated using the Sobolev bound (5.22). We decompose the nonlinear term as follows

$$
R_{2}\left(\varepsilon_{2}\right)-R_{1}\left(\varepsilon_{1}\right)=F_{1}+F_{2}
$$

where

$$
F_{1}=R_{2}\left(\varepsilon_{1}\right)-R_{1}\left(\varepsilon_{1}\right), \quad F_{2}=R_{2}\left(\varepsilon_{2}\right)-R_{2}\left(\varepsilon_{1}\right) .
$$

Using the expression of $R_{i}$ and $\left\|\varepsilon_{1}\right\|_{L^{\infty}} \lesssim|s|^{-\frac{1}{2}}$, we have

$$
\left|\left(F_{1}\right)_{y}\right|+\left|\left(F_{1}\right)_{y y}\right| \lesssim|b|\left(\left|\varepsilon_{1}\right|+\left|\left(\varepsilon_{1}\right)_{y}\right|+\left|\left(\varepsilon_{1}\right)_{y}^{2}\right|+\left|\left(\varepsilon_{1}\right)_{y y}\right|\right),
$$

and then by (5.23),

$$
\left(\left|\left(F_{1}\right)_{y}\right|+\left|\left(F_{1}\right)_{y y}\right|\right) e^{\frac{y}{4 B}} \lesssim|b|\left(\left|\varepsilon_{1}\right|+\left|\left(\varepsilon_{1}\right)_{y}\right|+\left|\left(\varepsilon_{1}\right)_{y y}\right|\right) e^{\frac{y}{4 B}}+\frac{|b|}{\sqrt{|s|}}\left|\left(\varepsilon_{1}\right)_{y}\right| .
$$

Thus, integrating by parts, and using Cauchy-Schwarz inequality and (5.22):

$$
\begin{aligned}
& \left|\int\left(F_{1}\right)_{y}\left[-\psi_{\bar{B}}^{\prime} \varepsilon_{y}+\psi_{\bar{B}}(L \varepsilon)+\left(\varphi_{\bar{B}}-\psi_{\bar{B}}\right) \varepsilon\right]\right| \\
& \lesssim \int\left(\left|\left(F_{1}\right)_{y}\right|+\left|\left(F_{1}\right)_{y y}\right|\right) e^{\frac{y}{2 \bar{B}}}\left(|\varepsilon|+\left|\varepsilon_{y}\right|\right) \sqrt{\varphi_{\bar{B}}^{\prime}} \\
& \lesssim|b| \int\left[\left(\left|\varepsilon_{1}\right|+\left|\left(\varepsilon_{1}\right)_{y}\right|+\left|\left(\varepsilon_{1}\right)_{y y}\right|\right) e^{\frac{y}{4 B}}+\left|\left(\varepsilon_{1}\right)_{y}\right|\right] e^{\frac{y}{4 \bar{B}}}\left(|\varepsilon|+\left|\varepsilon_{y}\right|\right) \sqrt{\varphi_{\bar{B}}^{\prime}} \\
& \lesssim \frac{|b|}{\sqrt{|s|}}\left(\int\left(\varepsilon_{y}^{2}+\varepsilon^{2}\right) \varphi_{\bar{B}}^{\prime}\right)^{\frac{1}{2}} \leq \frac{\mu_{1}}{100} \int\left(\varepsilon_{y}^{2}+\varepsilon^{2}\right) \varphi_{\bar{B}}^{\prime}+C \frac{b^{2}}{s} .
\end{aligned}
$$

We decompose $F_{2}$ as follows

$$
\begin{aligned}
F_{2} & =5\left(Q_{b_{2}}^{4}-Q^{4}\right) \varepsilon+10 Q_{b_{2}}^{3}\left(\varepsilon_{2}^{2}-\varepsilon_{1}^{2}\right)+10 Q_{b_{2}}^{2}\left(\varepsilon_{2}^{3}-\varepsilon_{1}^{3}\right)+5 Q_{b_{2}}\left(\varepsilon_{2}^{4}-\varepsilon_{1}^{4}\right) \\
& +\varepsilon_{2}^{5}-\varepsilon_{1}^{5} \\
& =\varepsilon\left[5\left(Q_{b_{2}}^{4}-Q^{4}\right)+10 Q_{b_{2}}^{3}\left(\varepsilon_{2}+\varepsilon_{1}\right)+10 Q_{b_{2}}^{2}\left(\varepsilon_{2}^{2}+\varepsilon_{1} \varepsilon_{2}+\varepsilon_{1}^{2}\right)\right. \\
& \left.+5 Q_{b_{2}}\left(\varepsilon_{2}^{3}+\varepsilon_{2}^{2} \varepsilon_{1}+\varepsilon_{2} \varepsilon_{1}^{2}+\varepsilon_{1}^{3}\right)+\left(\varepsilon_{2}^{4}+\varepsilon_{2}^{3} \varepsilon_{1}+\varepsilon_{2}^{2} \varepsilon_{1}^{2}+\varepsilon_{2} \varepsilon_{1}^{3}+\varepsilon_{1}^{4}\right)\right]
\end{aligned}
$$

Therefore, by suitable integration by parts, we have

$$
\begin{aligned}
& \left|\int\left(F_{2}\right)_{y}\left[-\psi_{\bar{B}}^{\prime} \varepsilon_{y}+\psi_{\bar{B}}\left(L \varepsilon-\varepsilon^{5}\right)+\left(\varphi_{\bar{B}}-\psi_{\bar{B}}\right) \varepsilon\right]\right| \\
& \lesssim\left|b_{2}\right| \int\left(\varepsilon_{y}^{2}+\varepsilon^{2}\right) \varphi_{\bar{B}}^{\prime}+\int\left(\varepsilon_{y}^{2}+\varepsilon^{2}\right) \varphi_{\bar{B}}\left(\left|\varepsilon_{1}\right|+\left|\varepsilon_{2}\right|\right) \\
& +\int\left(\varepsilon_{y}^{2}+\varepsilon^{2}\right) \psi_{\bar{B}} \sum_{i=1,2}\left(\left|\varepsilon_{i}\right|+\left|\left(\varepsilon_{i}\right)_{y}\right|+\left|\left(\varepsilon_{i}\right)_{y}^{2}\right|+\left|\left(\varepsilon_{i}\right)_{y y}\right|\right) .
\end{aligned}
$$

These terms are next treated as follows:

$$
C\left|b_{2}\right| \int\left(\varepsilon_{y}^{2}+\varepsilon^{2}\right) \varphi_{\bar{B}}^{\prime} \leq \frac{\mu_{1}}{100} \int\left(\varepsilon_{y}^{2}+\varepsilon^{2}\right) \varphi_{\bar{B}}^{\prime},
$$


and using (15.23),$\left\|\varepsilon_{i}\right\|_{L^{\infty}} \lesssim \frac{1}{\sqrt{|s|}}$ and the notation $y_{+}=\max (0, y)$ :

$$
\begin{aligned}
& \int\left(\varepsilon_{y}^{2}+\varepsilon^{2}\right) \varphi_{\bar{B}}\left(\left|\varepsilon_{1}\right|+\left|\varepsilon_{2}\right|\right) \\
& \lesssim\left(\left\|\varepsilon_{1}\left(1+\left|y_{+}\right|\right)\right\|_{L^{\infty}}+\left\|\varepsilon_{2}\left(1+\left|y_{+}\right|\right)\right\|_{L^{\infty}}\right) \int\left(\varepsilon_{y}^{2}+\varepsilon^{2}\right) \varphi_{\bar{B}}^{\prime} \\
& \leq \frac{\mu_{1}}{100} \int\left(\varepsilon_{y}^{2}+\varepsilon^{2}\right) \varphi_{\bar{B}}^{\prime} .
\end{aligned}
$$

Finally, using $\psi_{\bar{B}} \lesssim \varphi_{\frac{1}{B}}^{e^{\frac{y}{B}}}$ and $(15.23)$ :

$$
\begin{aligned}
& C \int\left(\varepsilon_{y}^{2}+\varepsilon^{2}\right) \psi_{\bar{B}} \sum_{i=1,2}\left(\left|\varepsilon_{i}\right|+\left|\left(\varepsilon_{i}\right)_{y}\right|+\left|\left(\varepsilon_{i}\right)_{y}^{2}\right|+\left|\left(\varepsilon_{i}\right)_{y y}\right|\right) \\
& \lesssim\left\|\left(\left|\varepsilon_{i}\right|+\left|\left(\varepsilon_{i}\right)_{y}\right|+\left|\left(\varepsilon_{i}\right)_{y}^{2}\right|+\left|\left(\varepsilon_{i}\right)_{y y}\right|\right) e^{\frac{y}{\bar{B}}}\right\|_{L^{\infty}} \int\left(\varepsilon_{y}^{2}+\varepsilon^{2}\right) \varphi_{\bar{B}}^{\prime} \\
& \leq \frac{\mu_{1}}{100} \int\left(\varepsilon_{y}^{2}+\varepsilon^{2}\right) \varphi_{\bar{B}}^{\prime} .
\end{aligned}
$$

The collection of above estimates yields the bound:

$$
\left|\int F_{y}\left[-\psi_{\bar{B}}^{\prime} \varepsilon_{y}+\psi_{\bar{B}}\left(L \varepsilon-\varepsilon^{5}\right)+\left(\varphi_{\bar{B}}-\psi_{\bar{B}}\right) \varepsilon\right]\right| \leq \frac{7 \mu_{1}}{100} \int\left(\varepsilon_{y}^{2}+\varepsilon^{2}\right) \varphi_{B}^{\prime}+C \frac{b^{2}}{|s|} .
$$

step 4 Proof of (5.18). We compute the time derivative of $\overline{\mathcal{F}_{2}}$ using (5.2):

$$
\begin{aligned}
\frac{1}{2} \frac{d \overline{\mathcal{F}_{2}}}{d s} & =\frac{\left(\lambda_{2}\right)_{s}}{2} \int y e^{\lambda_{2} y} \varepsilon^{2}+\int e^{\lambda_{2} y}\left(\partial_{s} \varepsilon\right) \varepsilon \\
& =\frac{\left(\lambda_{2}\right)_{s}}{2} \int y e^{\lambda_{2} y} \varepsilon^{2} \\
& +\int\left[(L \varepsilon)_{y}+\Gamma \Lambda Q_{b_{2}}+X\left(Q_{b_{2}}\right)_{y}+\frac{\left(\lambda_{2}\right)_{s}}{\lambda_{2}} \Lambda \varepsilon+E+F_{y}\right] e^{\lambda_{2} y} \varepsilon
\end{aligned}
$$

Since $\int \Lambda \varepsilon e^{\lambda_{2} y} \varepsilon=-\frac{1}{2} \lambda_{2} \int \varepsilon^{2} y e^{\lambda_{2} y}$, the scaling term cancels. By usual integrations by parts, we get

$$
\begin{aligned}
\frac{1}{2} \frac{d \overline{\mathcal{F}_{2}}}{d s} & =-\frac{3}{2} \lambda_{2} \int \varepsilon_{y}^{2} e^{\lambda_{2} y}-\frac{1}{2} \lambda_{2}\left(1-\lambda_{2}^{2}\right) \int \varepsilon^{2} e^{\lambda_{2} y} \\
& +\int\left(-10 Q^{3} Q_{y}+\frac{5}{2} \lambda_{2} Q^{4}\right) \varepsilon^{2} e^{\lambda_{2} y}+\int\left[\Gamma \Lambda Q_{b_{2}}+X\left(Q_{b_{2}}\right)_{y}+E+F_{y}\right] e^{\lambda_{2} y} \varepsilon
\end{aligned}
$$

Using $\lambda_{2} \sim \frac{1}{\sqrt{2|s|}}$ and the decay properties of $Q$, we get for $|s|$ large,

$$
\begin{aligned}
\frac{1}{2} \frac{d \overline{\mathcal{F}_{2}}}{d s} & \leq-\frac{1}{4 \sqrt{|s|}} \int\left(\varepsilon_{y}^{2}+\varepsilon^{2}\right) e^{\lambda_{2} y}+\int \varepsilon^{2} e^{-\frac{|y|}{10}} \\
& +\left|\int\left[\Gamma \Lambda Q_{b_{2}}+X\left(Q_{b_{2}}\right)_{y}+E+F_{y}\right] e^{\lambda_{2} y} \varepsilon\right| .
\end{aligned}
$$


40

Y. MARTEL, F. MERLE, AND P. RAPHAËL

Now, we estimate the remaining terms. First, from (5.9), and the definition of $Q_{b}$,

$$
\begin{aligned}
& \left|\Gamma \int \Lambda Q_{b_{2}} e^{\lambda_{2} y} \varepsilon\right|+\left|X \int\left(Q_{b_{2}}\right)_{y} e^{\lambda_{2} y} \varepsilon\right| \\
& \leq C\left(\left(\int \varepsilon^{2} e^{-\frac{|y|}{10}}\right)^{\frac{1}{2}}+\frac{|b|}{|s|}\right)\left(\left(\int \varepsilon^{2} e^{-\frac{|y|}{10}}\right)^{\frac{1}{2}}+\frac{\left|b_{2}\right|}{\sqrt{\lambda_{2}}}\left(\int \varepsilon^{2} e^{\lambda_{2} y}\right)^{\frac{1}{2}}\right) \\
& \leq \frac{\lambda_{2}}{100} \int \varepsilon^{2} e^{\lambda_{2} y}+C \int \varepsilon^{2} e^{-\frac{|y|}{10}}+C \frac{b^{2}}{s^{2}} .
\end{aligned}
$$

Second, we estimate terms coming from $E$. Since

$$
\int \Lambda \varepsilon_{1} \varepsilon e^{\lambda_{2} y}=-\int \varepsilon_{1} \Lambda \varepsilon e^{\lambda_{2} y}-\lambda_{2} \int y \varepsilon_{1} \varepsilon e^{\lambda_{2} y}
$$

we get

$$
\begin{aligned}
& \left|(\Gamma-b) \int \Lambda \varepsilon_{1} e^{\lambda_{2} y} \varepsilon\right| \\
& \lesssim\left(\left(\int \varepsilon^{2} e^{-\frac{|y|}{10}}\right)^{\frac{1}{2}}+|b|\right)\left(\int\left(\varepsilon_{y}^{2}+\varepsilon^{2}\right) e^{\lambda_{2} y}\right)^{\frac{1}{2}}\left(\int\left(1+y^{2}\right) \varepsilon_{1}^{2} e^{\lambda_{2} y}\right)^{\frac{1}{2}} .
\end{aligned}
$$

We estimate the term in $\varepsilon_{1}$ using Proposition 4.1, for some $0<\omega<\omega^{\prime}<\frac{1}{10}$, using $\lambda_{2} \geq \frac{19}{20} \lambda_{1}$ for $|s|$ large,

$$
\begin{aligned}
\int y^{2} \varepsilon_{1}^{2} e^{\lambda_{2} y} & \leq \int_{y<0} y^{2} \varepsilon_{1}^{2} e^{\lambda_{2} y}+\int_{y>0} y^{2} \varepsilon_{1}^{2} e^{\omega y} \\
& \lesssim \sup _{y<0}\left[y^{2} e^{\frac{1}{20} \lambda_{1} y}\right] \int_{y<0} \varepsilon_{1}^{2} e^{\frac{9}{10} \lambda_{1} y}+\int_{y>0} \varepsilon_{1}^{2} e^{\omega^{\prime} y} \\
& \lesssim \lambda_{1}^{-2}\left(\int_{y<0} \varepsilon_{1}^{2}\right)^{\frac{1}{10}}\left(\int_{y<0} \varepsilon_{1}^{2} e^{\lambda_{1} y}\right)^{\frac{9}{10}}+\int_{y>0} \varepsilon_{1}^{2} e^{\omega^{\prime} y} \lesssim|s|^{-\frac{9}{10}}
\end{aligned}
$$

we obtain

$$
\begin{aligned}
\left|(\Gamma-b) \int \Lambda \varepsilon_{1} e^{\lambda_{2} y} \varepsilon\right| & \lesssim|s|^{-\frac{9}{20}}\left(\left(\int \varepsilon^{2} e^{-\frac{|y|}{10}}\right)^{\frac{1}{2}}+|b|\right)\left(\int\left(\varepsilon_{y}^{2}+\varepsilon^{2}\right) e^{\lambda_{2} y}\right)^{\frac{1}{2}} \\
& \leq C \int \varepsilon^{2} e^{-\frac{|y|}{10}}+C \frac{|b|^{2}}{|s|^{\frac{2}{5}}}+\frac{\lambda_{2}}{100} \int\left(\varepsilon_{y}^{2}+\varepsilon^{2}\right) e^{\lambda_{2} y}
\end{aligned}
$$

For the second term coming from $E$, we use (5.24) and $\left|\Gamma_{1}\right| \lesssim \frac{1}{|s|}$, so that

$$
\begin{aligned}
\left|\Gamma_{1} \int \Lambda\left(Q_{b_{2}}-Q_{b_{1}}\right) \varepsilon e^{\lambda_{2} y}\right| & \lesssim \frac{|b|}{|s|}\left(\int_{y<0}|\varepsilon| e^{\lambda_{2} y}+\left(\int \varepsilon^{2} e^{-\frac{|y|}{10}}\right)^{\frac{1}{2}}\right) \\
& \lesssim \frac{|b|}{|s|}\left(|s|^{\frac{1}{4}}\left(\int_{y<0} \varepsilon^{2} e^{\lambda_{2} y}\right)^{\frac{1}{2}}+\left(\int \varepsilon^{2} e^{-\frac{|y|}{10}}\right)^{\frac{1}{2}}\right) \\
& \leq \frac{\lambda_{2}}{100} \int \varepsilon^{2} e^{\lambda_{2} y}+C \int \varepsilon^{2} e^{-\frac{|y|}{10}}+C \frac{|b|^{2}}{|s|} .
\end{aligned}
$$


For the last term in $E$, we use (5.25) and argue similarly:

$$
\begin{aligned}
& \left|\int\left(\Psi_{2}-\Psi_{1}\right) \varepsilon e^{\lambda_{2} y}\right| \\
& \lesssim\left(\int \varepsilon^{2} e^{-\frac{|y|}{10}}+\frac{\left(\int \varepsilon^{2} e^{-\frac{|y|}{10}}\right)^{\frac{1}{2}}}{|s|}+\frac{|b|}{\sqrt{|s|}}\right)\left(\int_{y<0}|\varepsilon| e^{\lambda_{2} y}+\left(\int \varepsilon^{2} e^{-\frac{|y|}{10}}\right)^{\frac{1}{2}}\right) \\
& \leq \frac{\lambda_{2}}{100} \int \varepsilon^{2} e^{\lambda_{2} y}+C \int \varepsilon^{2} e^{-\frac{|y|}{10}}+C \frac{|b|^{2}}{|s|} .
\end{aligned}
$$

Now, we estimate terms coming from $F$. Arguing as before, since $\left|X_{1}\right| \lesssim \frac{1}{|s|^{\frac{3}{2}}}$ and using (5.24), we obtain

$$
\left|X_{1} \int\left(Q_{b_{2}}-Q_{b_{1}}\right)_{y} \varepsilon e^{\lambda_{2} y}\right| \lesssim \frac{\lambda_{2}}{100} \int \varepsilon^{2} e^{\lambda_{2} y}+\int \varepsilon^{2} e^{-\frac{|y|}{10}}+\frac{|b|^{2}}{|s|} .
$$

Next, since $\left|X_{2}\right| \lesssim \frac{1}{|s|^{\frac{3}{2}}}, \lambda_{2} \lesssim|s|^{-\frac{1}{2}}$, by integration by parts

$$
\left|X_{2} \int \varepsilon_{y} \varepsilon e^{\lambda_{2} y}\right| \lesssim \frac{1}{s^{2}} \int \varepsilon^{2} e^{\lambda_{2} y} \leq \frac{\lambda_{2}}{100} \int \varepsilon^{2} e^{\lambda_{2} y}
$$

Then, by integration by parts, (5.9) and (4.11),

$$
\begin{aligned}
\left|X \int\left(\partial_{y} \varepsilon_{1}\right) \varepsilon e^{\lambda_{2} y}\right| & \lesssim\left(\left(\int \varepsilon^{2} e^{-\frac{|y|}{10}}\right)^{\frac{1}{2}}+\frac{|b|}{|s|}\right)\left(\int \varepsilon_{1}^{2} e^{\lambda_{2} y}\right)^{\frac{1}{2}}\left(\int\left(\varepsilon_{y}^{2}+\varepsilon^{2}\right) e^{\lambda_{2} y}\right)^{\frac{1}{2}} \\
& \lesssim \frac{\lambda_{2}}{100} \int\left(\varepsilon_{y}^{2}+\varepsilon^{2}\right) e^{\lambda_{2} y}+\int \varepsilon^{2} e^{-\frac{|y|}{10}}+\frac{|b|^{2}}{|s|}
\end{aligned}
$$

Finally, we decompose $R_{2}\left(\varepsilon_{2}\right)-R_{1}\left(\varepsilon_{1}\right)$ as follows, using $\left\|\varepsilon_{i}\right\|_{L^{\infty}} \lesssim|s|^{-\frac{1}{2}}$ and $\left|b_{i}\right| \sim$ $|s|^{-1}$,

$$
\begin{aligned}
\left|R_{2}\left(\varepsilon_{2}\right)-R_{1}\left(\varepsilon_{1}\right)\right| & \leq\left|R_{2}\left(\varepsilon_{2}\right)-R_{2}\left(\varepsilon_{1}\right)\right|+\left|R_{2}\left(\varepsilon_{1}\right)-R_{1}\left(\varepsilon_{1}\right)\right| \\
& \lesssim|\varepsilon|\left(\left|b_{2}\right|+e^{-\frac{|y|}{10}}\right)+|b|\left|\varepsilon_{1}\right| \lesssim|s|^{-1}|\varepsilon|+|\varepsilon| e^{-\frac{|y|}{10}}+|b|\left|\varepsilon_{1}\right|,
\end{aligned}
$$

and thus, using also (4.11), we estimate the last term coming from $F$ as follows:

$$
\begin{aligned}
& \left|\int\left(R_{2}\left(\varepsilon_{2}\right)-R_{1}\left(\varepsilon_{1}\right)\right)\left(\varepsilon_{y}+\lambda_{2} \varepsilon\right) e^{\lambda_{2} y}\right| \\
& \lesssim \frac{1}{|s|} \int\left(\varepsilon_{y}^{2}+\varepsilon^{2}\right) e^{\lambda_{2} y}+\int \varepsilon^{2} e^{-\frac{|y|}{10}}+|b|\left(\int \varepsilon_{1}^{2} e^{\lambda_{2} y}\right)^{\frac{1}{2}}\left(\int \varepsilon^{2} e^{\lambda_{2} y}\right)^{\frac{1}{2}} \\
& \lesssim \frac{\lambda_{2}}{100} \int\left(\varepsilon_{y}^{2}+\varepsilon^{2}\right) e^{\lambda_{2} y}+\int \varepsilon^{2} e^{-\frac{|y|}{10}}+\frac{|b|^{2}}{|s|} .
\end{aligned}
$$

The collection of above bounds yields (5.18).

5.4. Conclusion. We are now in position to conclude the proof of uniqueness (5.1).

First, recall that the following estimates from Proposition 4.1 as $s \rightarrow-\infty$ :

$$
\overline{\mathcal{N}} \lesssim \frac{1}{|s|^{3}}, \quad\left|J_{2}\right| \lesssim \overline{\mathcal{N}}^{\frac{1}{2}} \lesssim \frac{1}{|s|^{\frac{3}{2}}}, \quad b=o\left(\frac{1}{|s|^{2}}\right) .
$$


Moreover, we estimate from (4.11), 4.12):

$$
\begin{aligned}
\int e^{\lambda_{2} y} \varepsilon^{2} & \lesssim \int e^{\lambda_{2} y} \varepsilon_{1}^{2}+\int e^{\lambda_{2} y} \varepsilon_{2}^{2} \lesssim \int e^{\lambda_{2} y} \varepsilon_{1}^{2}+\frac{1}{s^{2}} \\
& \lesssim \int_{y<0} e^{-\lambda_{2}|y|} \varepsilon_{1}^{2}+\int_{y>0} e^{\lambda_{2} y} \varepsilon_{1}^{2}+\frac{1}{s^{2}} \\
& \lesssim \int_{y<0} e^{-\frac{9}{10} \lambda_{1}|y|} \varepsilon_{1}^{2}+\int_{y>0} e^{\frac{y}{10}} \varepsilon_{1}^{2}+\frac{1}{s^{2}} \lesssim\left(\int e^{-\lambda_{1}|y|} \varepsilon_{1}^{2}\right)^{\frac{9}{10}}+\frac{1}{s^{2}} \\
& \lesssim \frac{1}{s^{\frac{9}{5}}} .
\end{aligned}
$$

This yields in particular from (5.7) the bound:

$$
\mathcal{F} \lesssim \frac{1}{s^{2+\frac{3}{10}}}
$$

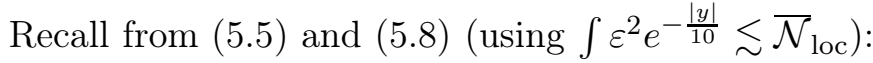

$$
\begin{gathered}
\frac{d}{d s}\left\{s^{2}\left(b+\frac{J_{2}}{2 s}\right)\right\} \lesssim s^{2} \overline{\mathcal{N}}_{\text {loc }}+|s|^{\frac{1}{2}}|b|+|s|^{\frac{1}{2}} \overline{\mathcal{N}}^{\frac{1}{2}}, \\
\frac{d}{d s}\left(s^{2} \mathcal{F}\right)+\mu s^{2} \overline{\mathcal{N}}_{\text {loc }} \lesssim|s|^{\frac{11}{10}} b^{2} .
\end{gathered}
$$

It follows that for $K_{0}>0$ large enough, using also (5.27),

$$
\frac{d}{d s}\left\{s^{2}\left(b+\frac{J_{2}}{2 s}+K_{0} \mathcal{F}\right)\right\} \lesssim|s|^{\frac{1}{2}}|b|+|s|^{\frac{1}{2}} \overline{\mathcal{N}}^{\frac{1}{2}}+s^{\frac{11}{10}} b^{2} \lesssim|s|^{\frac{1}{2}}|b|+|s|^{\frac{1}{2}} \overline{\mathcal{N}}^{\frac{1}{2}}
$$

We now observe from (5.27), (5.28) the a priori bound:

$$
s^{2}\left(|b|+\frac{\left|J_{2}\right|}{|s|}+\mathcal{F}\right) \rightarrow 0 \text { as } s \rightarrow-\infty .
$$

We then integrate (5.29) on $(-\infty, s]$ :

$s^{2} \mathcal{F}(s) \lesssim \int_{-\infty}^{s}\left|s^{\prime}\right|^{\frac{11}{10}} b^{2}\left(s^{\prime}\right) d s^{\prime} \lesssim \int_{-\infty}^{s}\left(s^{\prime}\right)^{4} b^{2}\left(s^{\prime}\right) \frac{d s^{\prime}}{\left|s^{\prime}\right|^{2+\frac{9}{10}}} \lesssim \frac{1}{|s|^{\frac{19}{10}}}\left(\sup _{(-\infty, s]}\left(\left(s^{\prime}\right)^{2}\left|b\left(s^{\prime}\right)\right|\right)^{2}\right.$, so that

$$
|s| \mathcal{F}^{\frac{1}{2}}(s) \lesssim \frac{1}{|s|^{\frac{19}{20}}} \sup _{(-\infty, s]}\left(s^{\prime}\right)^{2}\left|b\left(s^{\prime}\right)\right| .
$$

Next, by integration of (5.30) on $(-\infty, s]$ and (5.7) and (5.27),

$$
\begin{aligned}
s^{2}|b(s)| & \lesssim|s|\left|J_{2}\right|+s^{2} \mathcal{F}+\int_{-\infty}^{s}\left(\left|s^{\prime}\right|^{\frac{1}{2}}|b|+\left|s^{\prime}\right|^{\frac{1}{2}} \overline{\mathcal{N}}^{\frac{1}{2}}\right) d s^{\prime} \\
& \lesssim|s| \overline{\mathcal{N}}^{\frac{1}{2}}+s^{2} \mathcal{F}+\int_{-\infty}^{s}|b|\left|s^{\prime}\right|^{2} \frac{d s^{\prime}}{\left|s^{\prime}\right|^{\frac{3}{2}}}+\int_{-\infty}^{s}\left|s^{\prime}\right|^{\frac{7}{4}} \overline{\mathcal{N}}^{\frac{1}{2}} \frac{d s^{\prime}}{\left|s^{\prime}\right|^{\frac{5}{4}}} \\
& \lesssim \frac{1}{|s|^{\frac{1}{4}}} \sup _{(-\infty, s]}\left\{\left(s^{\prime}\right)^{2}\left|b\left(s^{\prime}\right)\right|+\left|s^{\prime}\right|^{\frac{7}{4}} \mathcal{F}^{\frac{1}{2}}\left(s^{\prime}\right)\right\} .
\end{aligned}
$$

Putting together (5.31) and (5.32), we get

$$
s^{2}|b(s)|+|s|^{\frac{7}{4}} \mathcal{F}^{\frac{1}{2}}(s) \lesssim \frac{1}{|s|^{\frac{1}{5}}} \sup _{(-\infty, s]}\left\{\left(s^{\prime}\right)^{2}\left|b\left(s^{\prime}\right)\right|+\left(s^{\prime}\right)^{\frac{7}{4}} \mathcal{F}^{\frac{1}{2}}\left(s^{\prime}\right)\right\} .
$$

This give immediately for $|s|$ large, by (5.7),

$$
|b(s)|+\overline{\mathcal{N}}(s)=0 \text { and thus } \varepsilon(s, y) \equiv 0 .
$$


Therefore, for some $t>0, u_{2}(t)$ is a rescaled and translated of $S(t)$ and thus for all time by uniqueness of the Cauchy problem in $H^{1}$. This concludes the proof of (5.1) and Theorem 1.3 .

\section{Description of the (Exit) scenario}

This section is devoted to the proof of Theorem 1.4, The argument relies first on an extension of the compactness argument of section 3 and second on the uniqueness up to symmetries of the minimal mass blow up solution.

6.1. Reduction of the proof. Theorem 1.4 is a direct consequence of the following proposition which describes the defocusing bubble in the (Exit) regime at the exit time.

Proposition 6.1 (Compactness of sequences of solutions at the (Exit) time). There exists a small universal constant $\alpha^{*}>0$ such that the following holds. Let $\left(u_{n}(0)\right)$ be a sequence in $H^{1}$ satisfying:

(1) $u_{n}(0) \in \mathcal{A}$;

(2) $\left\|u_{n}(0)-Q\right\|_{H^{1}} \leq \frac{1}{n}$;

(3) the solution $u_{n} \in \mathcal{C}\left(\left[0, T_{n}\right), H^{1}\right)$ of (1.1) corresponding to $\left(u_{n}(0)\right)_{n \geq 1}$ satisfies the (Exit) scenario, i.e. for all $n$ large enough,

$$
t_{n}^{*}=\sup \left\{t>0 \text { such that } \forall t^{\prime} \in[0, t], u_{n}\left(t^{\prime}\right) \in \mathcal{T}_{\alpha^{*}}\right\}<T_{n} .
$$

Then, there exists $\sigma^{*}=\sigma^{*}\left(\alpha^{*}\right)$ (independent of the sequence $u_{n}$ ) such that

$$
\lambda_{n}^{\frac{1}{2}}\left(t_{n}^{*}\right) u_{n}\left(t_{n}^{*}, \lambda_{n}\left(t_{n}^{*}\right) \cdot+x_{n}\left(t_{n}^{*}\right)\right) \rightarrow \lambda_{S}^{\frac{1}{2}}\left(\sigma^{*}\right) S\left(\sigma^{*}, \lambda_{S}\left(\sigma^{*}\right) \cdot+x_{S}\left(\sigma^{*}\right)\right) \quad \text { in } L^{2}
$$

as $n \rightarrow+\infty$.

6.2. Proof of Proposition 6.1. The strategy of the proof is similar to the proof of existence of Theorem 1.3 in section 3 . However the initial data in section 3 are well prepared and in particular generate $H^{1}$ bounded sequences after renormalization, see (3.6). Here the $H^{1}$ bound is lost, and one needs to invoke a concentration compactness argument in the critical $L^{2}$ space for sequences of solutions to (1.1) and uniform local estimates to recover a non trivial weak limit.

step 1 Renormalization. Let $C^{*}>0$ be the universal constant in (2.57) of Lemma 2.10, Let $t_{n}^{*}$ be the exit time (6.1), and consider the decomposition of $u_{n}(t)$ on $\left[0, t_{n}^{*}\right]$ given by Lemma [2.6. It follows from the proof of Theorem 1.2 in [33], Section 4.3, that there exists a time $0 \leq t_{1, n}^{*}<t_{n}^{*}$ such that

$$
b_{n}\left(t_{1, n}^{*}\right) \leq-C^{*} \int\left(\left(\partial_{y} \varepsilon_{n}\right)^{2}\left(t_{1, n}^{*}\right) \psi_{B}+\varepsilon_{n}^{2}\left(t_{1, n}^{*}\right) \varphi_{B}^{\prime}\right),
$$

and

$$
\lambda_{n}\left(t_{1, n}^{*}\right) \approx 1, \quad\left|b_{n}\left(t_{1, n}^{*}\right)\right|+\mathcal{N}_{n}\left(t_{1, n}^{*}\right) \rightarrow 0 \quad \text { as } n \rightarrow \infty,
$$

where $\mathcal{N}_{n}$ denotes the quantity $\mathcal{N}$ defined in (2.46) for $u_{n}$. This time corresponds to when the (Exit) regime is decided $\left(b_{n}\right.$ is negative and becomes predominant in the sense (6.3) $)$, and it is proved in [33] that such a time $t_{1, n}^{*}$ can be chosen so that the solution has moved only $\delta\left(\left\|u_{n}(0)-Q\right\|_{H^{1}}\right)$ far from the initial data (see equation (4.37) in [33]), which implies (6.4) in the present situation (since $\left\|u_{n}(0)-Q\right\| \rightarrow 0$ as $n \rightarrow \infty)$.

Recall also from [33] that $u_{n}(t)$ satisfies $(\mathrm{H} 1),(\mathrm{H} 2)$ and $(\mathrm{H} 3)$ on $\left[0, t_{n}^{*}\right]$. 
Define

$$
\forall \tau \in\left[\tau_{n}^{*}, 0\right], \quad t_{\tau}=t_{n}^{*}+\tau \lambda_{n}^{3}\left(t_{n}^{*}\right), \quad \tau_{n}^{*}=-\frac{t_{n}^{*}-t_{1, n}^{*}}{\lambda_{n}^{3}\left(t_{n}^{*}\right)}
$$

and consider on $\left[\tau_{n}^{*}, 0\right]$ the renormalized solution $v_{n}(\tau)$ at the exit time $t_{n}^{*}$,

$$
\begin{aligned}
v_{n}(\tau, x)= & \lambda_{n}^{\frac{1}{2}}\left(t_{n}^{*}\right) u_{n}\left(t_{\tau}, \lambda_{n}\left(t_{n}^{*}\right) x+x\left(t_{n}^{*}\right)\right) \\
= & \frac{\lambda_{n}^{\frac{1}{2}}\left(t_{n}^{*}\right)}{\lambda_{n}^{\frac{1}{2}}\left(t_{\tau}\right)}\left(Q_{b_{n}\left(t_{\tau}\right)}+\varepsilon_{n}\right)\left(t_{\tau}, \frac{\lambda_{n}\left(t_{n}^{*}\right)}{\lambda_{n}\left(t_{\tau}\right)} x+\frac{x_{n}\left(t_{n}^{*}\right)-x_{n}\left(t_{\tau}\right)}{\lambda_{n}\left(t_{\tau}\right)}\right) .
\end{aligned}
$$

Then $v_{n}$ is solution of (1.1) and belongs to the $L^{2}$ tube $\mathcal{T}_{\alpha^{*}}$ for $\tau \in\left[\tau_{n}^{*}, 0\right]$. Moreover, its decomposition $\left(\lambda_{v_{n}}, x_{v_{n}}, \varepsilon_{v_{n}}\right)$ satisfies on $\left[\tau_{n}^{*}, 0\right]$ :

$$
\lambda_{v_{n}}(\tau)=\frac{\lambda_{n}\left(t_{\tau}\right)}{\lambda_{n}\left(t_{n}^{*}\right)}, x_{v_{n}}(\tau)=\frac{x_{n}\left(t_{\tau}\right)-x_{n}\left(t_{n}^{*}\right)}{\lambda_{n}\left(t_{n}^{*}\right)}, b_{v_{n}}(\tau)=b_{n}\left(t_{\tau}\right), \varepsilon_{v_{n}}(\tau)=\varepsilon_{n}\left(t_{\tau}\right) .
$$

step 2 Preliminary estimates on the renormalized sequence. We claim:

Lemma 6.2. There exist $b^{*}, \tau^{*}$ such that, possibly extracting a subsequence,

$$
\begin{gathered}
b_{n}\left(t_{n}^{*}\right) \rightarrow-b^{*}, \quad\left(\alpha^{*}\right)^{2} \lesssim b^{*} \leq \delta\left(\alpha^{*}\right), \\
\tau_{n}^{*}=-\frac{t_{n}^{*}}{\lambda_{n}^{3}\left(t_{n}^{*}\right)} \rightarrow-\tau^{*}, \quad \tau^{*} b^{*} \approx 1 .
\end{gathered}
$$

Moreover, for all $n$ large, $\tau \in\left[\tau_{n}^{*}, 0\right]$,

$$
\begin{gathered}
\lambda_{v_{n}}(0)=1, \quad x_{v_{n}}(0)=0, \\
\left|b_{v_{n}}(\tau)\right|+\mathcal{N}_{v_{n}}(\tau)+\left\|\varepsilon_{v_{n}}(\tau)\right\|_{L^{2}} \lesssim \delta\left(\alpha^{*}\right), \\
\int_{\tau_{1, n}^{*}}^{\tau_{n}^{*}}\left[\int\left(\left(\partial_{y} \varepsilon_{v_{n}}\right)^{2}+\varepsilon_{v_{n}}^{2}\right) \frac{\varphi_{B}}{1+y_{+}^{2}}+\left|b_{v_{n}}\right|^{4}\right] \frac{d \tau}{\lambda_{v_{n}}^{5}(\tau)} \lesssim \delta\left(\alpha^{*}\right), \\
\frac{\left|b_{v_{n}}\left(\tau_{n}^{*}\right)\right|}{\lambda_{v_{n}}^{2}\left(\tau_{n}^{*}\right)} \lesssim \delta\left(\alpha^{*}\right), \quad \lambda_{v_{n}}\left(\tau_{n}^{*}\right)=\frac{1}{\lambda_{n}\left(t_{n}^{*}\right)} \rightarrow 0 \quad \text { as } n \rightarrow+\infty, \\
\left(\lambda_{0, v_{n}}\right)_{\tau}(\tau) \approx b^{*}, \\
x_{v_{n}}\left(\tau_{n}^{*}\right) \lesssim-\frac{1}{b^{*} \lambda_{v_{n}}\left(\tau_{n}^{*}\right)} \rightarrow-\infty \quad \text { as } n \rightarrow+\infty .
\end{gathered}
$$

(Recall that $\lambda_{0}$ is defined in Lemma 2.10. Here, $\lambda_{0, v_{n}}$ denotes this quantity for $v_{n}$. Similarly, $\mathcal{N}_{v_{n}}$ denotes the quantity $\mathcal{N}$ for $v_{n}$. As usual $y_{+}=\max (0, y)$.)

Proof of Lemma 6.2. Arguing as in the proof of Lemma 3.1, using conservation of mass and energy of $u_{n}(t)$, we first obtain

$$
\left(\alpha^{*}\right)^{2} \lesssim-b_{n}\left(t_{n}^{*}\right) \lesssim \delta\left(\alpha^{*}\right)
$$

Next, using (2.57) in Lemma 2.10 on $\left[t_{1, n}^{*}, t_{n}^{*}\right]$, and (6.3), (6.4), one obtains

$$
-\frac{b_{n}(t)}{\lambda_{n}^{2}(t)} \approx-b_{n}\left(t_{1, n}^{*}\right)
$$

and thus, by (6.16),

$$
\frac{\left(\alpha^{*}\right)^{2}}{\left|b_{n}\left(t_{1, n}^{*}\right)\right|} \lesssim \lambda_{n}^{2}\left(t_{n}^{*}\right) \lesssim \frac{\delta\left(\alpha^{*}\right)}{\left|b_{n}\left(t_{1, n}^{*}\right)\right|}
$$


which implies $\frac{\left|b_{v_{n}}\left(\tau_{n}^{*}\right)\right|}{\lambda_{v_{n}}^{2}\left(\tau_{n}^{*}\right)} \lesssim \delta\left(\alpha^{*}\right)$. Next, by definition of $t_{n}^{*},\left\|\varepsilon_{v_{n}}(\tau)\right\|_{L^{2}}=\left\|\varepsilon_{n}\left(t_{\tau}\right)\right\|_{L^{2}} \leq$ $\delta\left(\alpha^{*}\right)$. By (2.59) and (6.4), for $n$ large,

$$
\begin{aligned}
& \mathcal{N}_{n}(t)+\int_{t_{1, n}^{*}}^{t_{n}^{*}}\left[\int\left(\left(\partial_{y} \varepsilon_{n}\right)^{2}+\varepsilon_{n}^{2}\right) \varphi_{B}^{\prime}\right] \frac{d t}{\lambda_{n}^{3}} \\
& \lesssim \mathcal{N}_{n}\left(t_{1, n}^{*}\right)+\left|b_{n}(t)\right|^{3}+\left|b_{n}\left(t_{1, n}^{*}\right)\right|^{3} \leq \delta\left(\alpha^{*}\right) .
\end{aligned}
$$

Now, we use Lemma 4.3 in [33] to obtain a slightly different estimate. From (4.12) in [33], with $i=1$, using the definition of $\varphi_{i, B}$ in page 84, and then using (6.3), we obtain

$$
\begin{aligned}
& \int_{t_{1, n}^{*}}^{t_{n}^{*}}\left[\int\left(\left(\partial_{y} \varepsilon_{n}\right)^{2}+\varepsilon_{n}^{2}\right) \frac{\varphi_{B}}{1+y_{+}^{2}}+\left|b_{n}\right|^{4}\right] \frac{d t}{\lambda_{n}^{5}} \\
& \lesssim \frac{\int\left(\left(\partial_{y} \varepsilon_{n}\right)^{2}\left(t_{1, n}^{*}\right) \psi_{B}+\varepsilon_{n}^{2}\left(t_{1, n}^{*}\right) \varphi_{B}^{\prime}\right)}{\lambda_{n}^{2}\left(t_{1, n}^{*}\right)}+\frac{\left|b_{n}\left(t_{n}^{*}\right)\right|^{3}}{\lambda_{n}^{2}\left(t_{n}^{*}\right)}+\frac{\left|b_{n}\left(t_{1, n}^{*}\right)\right|^{3}}{\lambda_{n}^{2}\left(t_{1, n}^{*}\right)} \lesssim \frac{\left|b_{n}\left(t_{1, n}^{*}\right)\right|}{\lambda_{n}^{2}\left(t_{1, n}^{*}\right)} \\
& \lesssim \delta\left(\alpha^{*}\right) .
\end{aligned}
$$

Moreover, by (2.58) and (6.17),

$$
\left(\lambda_{0 n}\right)_{t}(t) \approx \frac{\left|b_{n}\left(t_{n}^{*}\right)\right|}{\lambda_{n}^{2}\left(t_{n}^{*}\right)} .
$$

By definition of $v_{n}$, we obtain (6.10) -(6.14) from the above estimates.

Now, we prove (6.15). Integrating the estimate of $\left(\lambda_{0, v_{n}}\right)_{\tau}$, we obtain

$$
\lambda_{0, v_{n}}(\tau)-\lambda_{0, v_{n}}\left(\tau_{n}^{*}\right) \approx b^{*}\left(\tau-\tau_{n}^{*}\right)
$$

Finally, since $\left(x_{v_{n}}\right)_{\tau} \approx \frac{1}{\lambda_{0, v_{n}}^{2}}$, we obtain by integration of $\left[\tau_{n}^{*}, 0\right]$ and $x_{v_{n}}(0)=0$, for $n$ large,

$$
-x_{v_{n}}\left(\tau_{n}^{*}\right)=x_{v_{n}}(0)-x_{v_{n}}\left(\tau_{n}^{*}\right) \approx \frac{1}{b^{*}} \frac{1}{\lambda_{v_{n}}\left(\tau_{n}^{*}\right)}
$$

and (6.15) is proved.

step 3 Monotonicity estimates. We now claim the following bound on $v_{n}$ which will allow us to recover $H^{1}$ bounds in the limit:

$$
\int_{x>-\lambda_{n}^{2}\left(t_{n}^{*}\right)}\left(\partial_{x} v_{n}\right)^{2}(0, x) d x \lesssim 1
$$

In fact, we prove the following estimate on $\varepsilon_{v_{n}}(0)$ which, together with Lemma 6.2 and $\lambda_{v_{n}}(0)=1$ implies (6.22)

$$
\int_{y>-2 \lambda_{n}^{2}\left(t_{n}^{*}\right)}\left(\partial_{y} \varepsilon_{v_{n}}\right)^{2}(0, y) d y \lesssim \delta\left(\alpha^{*}\right)
$$

Proof of (6.23). For $\tau \in\left[\tau_{n}^{*}, 0\right]$, let $s=-\int_{\tau}^{0} \frac{d \tau}{\lambda_{v_{n}}^{3}(\tau)}$ the rescaled time for $v_{n}$ and $s_{n}^{*}=-\int_{\tau_{n}^{*}}^{0} \frac{d \tau}{\lambda_{v_{n}}^{3}(\tau)}$. We perform monotonicity estimates on $\varepsilon_{v_{n}}$ to complement the ones obtained in (2.59). We define $\phi \in \mathcal{C}^{\infty}(\mathbb{R})$, such that

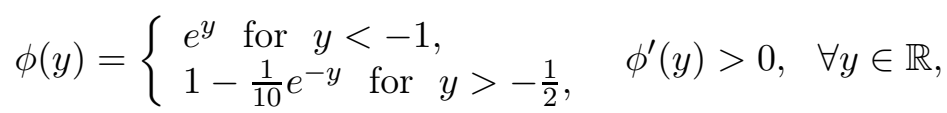

and we consider $\psi$ defined as in (2.45). Let

$$
\phi_{B}(s, y)=\phi\left(\frac{y+\frac{1}{2}\left(s-s_{n}^{*}\right)}{B}\right), \quad \tilde{\phi}_{B}(s, y)=\psi\left(\frac{y+\frac{1}{2}\left(s-s_{n}^{*}\right)}{B}\right),
$$


and

$$
\begin{aligned}
\mathcal{F}_{B}(s)=\frac{1}{\lambda_{v_{n}}^{2}(s)} \int\left[\left(\partial_{y} \varepsilon_{v_{n}}\right)^{2} \tilde{\phi}_{B}\right. & +\frac{\lambda_{v_{n}}^{2}(s)}{\lambda_{0, v_{n}}^{2}(s)} \varepsilon_{v_{n}}^{2} \phi_{B} \\
& \left.-\frac{1}{3}\left(\left(\varepsilon_{v_{n}}+Q_{b}\right)^{6}-Q_{b}^{6}-6 \varepsilon_{v_{n}} Q_{b}^{5}\right) \tilde{\phi}_{B}\right](s, y) d y .
\end{aligned}
$$

We claim the following estimates proved in Appendix B

Lemma 6.3. For B large enough,

$$
\begin{aligned}
\frac{d}{d s} \mathcal{F}_{B} \leq & -\frac{1}{4} \frac{1}{\lambda_{v_{n}}^{2}} \int\left(\left(\partial_{y} \varepsilon_{v_{n}}\right)^{2}+\varepsilon_{v_{n}}^{2}\right) \phi_{B}^{\prime} \\
+ & \frac{C}{\lambda_{v_{n}}^{2}} \int\left(\left(\partial_{y} \varepsilon_{v_{n}}\right)^{2}+\varepsilon_{v_{n}}^{2}\right) \frac{\varphi_{B}}{1+y_{+}^{2}}+\frac{C}{\lambda_{v_{n}}^{2}}\left|b_{v_{n}}\right|^{4} \\
\mathcal{F}_{B} & \approx \frac{1}{\lambda_{v_{n}}^{2}} \int\left(\partial_{y} \varepsilon_{v_{n}}\right)^{2} \tilde{\phi}_{B}+\varepsilon_{v_{n}}^{2} \phi_{B}
\end{aligned}
$$

We integrate (6.25) on $\left[\tau_{n}^{*}, 0\right]$ and then use (6.20) and Lemma 6.2

$$
\begin{aligned}
\mathcal{F}_{B}(0) & \leq \mathcal{F}_{B}\left(\tau_{n}^{*}\right)+C \int_{\tau_{n}^{*}}^{0}\left(\int\left(\left(\partial_{y} \varepsilon_{v_{n}}\right)^{2}+\varepsilon_{v_{n}}^{2}\right) \frac{\varphi_{B}}{1+y_{+}^{2}}+\left|b_{v_{n}}\right|^{4}\right) \frac{d \tau}{\lambda_{v_{n}}^{5}(\tau)} \\
& \lesssim \mathcal{N}_{v_{n}}\left(\tau_{n}^{*}\right)+\frac{\left|b_{v_{n}}\left(\tau_{n}^{*}\right)\right|^{3}}{\lambda_{v_{n}}^{2}\left(\tau_{n}^{*}\right)}+\left|b_{v_{n}}(0)\right|^{3} \lesssim \delta\left(\alpha^{*}\right) .
\end{aligned}
$$

And thus, by (6.26),$\lambda_{v_{n}}(0)=1$ and the definition of $\phi_{B}, \tilde{\phi}_{B}$, since

$$
s_{n}^{*}=-\int_{\tau_{n}^{*}}^{0} \frac{d \tau}{\lambda_{v_{n}}^{3}(\tau)} \approx-\int_{\tau_{n}^{*}}^{0} \frac{d \tau}{\left(\lambda_{v_{n}}\left(\tau_{n}^{*}\right)+b^{*}\left(\tau-\tau_{n}^{*}\right)\right)^{3}} \approx-\frac{1}{b^{*}} \frac{1}{\lambda_{v_{n}}^{2}\left(\tau_{n}^{*}\right)},
$$

we finally obtain

$$
\int_{y>-2 \lambda_{v_{n}}^{-2}\left(\tau_{n}^{*}\right)}\left(\partial_{y} \varepsilon_{v_{n}}\right)^{2} \leq \int_{y>\frac{1}{2} s_{n}^{*}}\left(\partial_{y} \varepsilon_{v_{n}}\right)^{2} \lesssim \delta\left(\alpha^{*}\right) .
$$

step 4 Extraction of the limit. Since $\left\|v_{n}(0)-Q\right\|_{L^{2}} \leq \delta\left(\alpha^{*}\right)$, there exists $v(0) \in$ $L^{2}$ and a subsequence still denoted $\left(v_{n}(0)\right)$ such that

$$
v_{n}(0) \rightarrow v(0) \text { in } L^{2} \text { weak as } n \rightarrow \infty .
$$

Moreover, by properties of the weak convergence,

$$
\|v(0)\|_{L^{2}} \leq\|Q\|_{L^{2}}, \quad\|v(0)-Q\|_{L^{2}} \leq \delta\left(\alpha^{*}\right)
$$

and since $\lambda_{n}\left(t_{n}^{*}\right) \rightarrow \infty$, it follows from (6.22) that

$$
v(0) \in H^{1} \text {. }
$$

Let $\delta_{0}>0$ small enough, $\delta_{0}<\delta$ where $\delta$ is defined in Theorem 2.1 (i). We consider $\alpha^{*}$ small enough, but universal, such that

$$
\left\|v_{n}(0)-v(0)\right\|_{L^{2}} \leq \frac{\delta_{0}}{2}
$$

In order to exhibit a non trivial weak limit, we decompose the sequence $\left(v_{n}(0)-v(0)\right)$ into profiles according to Lemma 2.3: there exist

$$
U_{n}^{j}(0)=e^{-t_{n}^{j} \partial_{x}^{3}}\left(g_{n}^{j}\left[\operatorname{Re}\left(e^{i x \xi_{n}^{j} \lambda_{n}^{j}} \phi^{j}\right)\right]\right)
$$


and $w_{n}^{J}(0) \in L^{2}$ such that (up to a subsequence)

$$
\begin{gathered}
v_{n}(0)-v(0)=\sum_{j=1}^{J} U_{n}^{j}(0)+w_{n}^{J}(0), \quad \lim _{J \rightarrow \infty} \limsup _{n \rightarrow \infty}\left\|e^{-t \partial_{x}^{3}} w_{n}^{J}(0)\right\|_{L_{x}^{5} L_{t}^{10}(\mathbb{R} \times \mathbb{R})}=0 \\
\left\|v_{n}(0)-v(0)\right\|_{L^{2}}^{2}-\sum_{j=1}^{J}\left\|U_{n}^{j}(0)\right\|_{L^{2}}^{2}-\left\|w_{n}^{J}(0)\right\|_{L^{2}}^{2}=o_{n}(1)
\end{gathered}
$$

Moreover, by weak convergence $v_{n}(0) \rightarrow v(0)$, we have

$$
\int v(0)\left(v_{n}(0)-v(0)\right)=o_{n}(1)
$$

and thus,

$$
\left\|v_{n}(0)\right\|_{L^{2}}^{2}-\|v(0)\|_{L^{2}}^{2}-\sum_{j=1}^{J}\left\|U_{n}^{j}(0)\right\|_{L^{2}}^{2}-\left\|w_{n}^{J}(0)\right\|_{L^{2}}^{2}=o_{n}(1)
$$

In particular, by (6.30) and (6.32),$v(0)$ is interpreted as the first profile $U^{0}$ of the decomposition of $v_{n}(0)$ with $g_{n}^{0}=g_{1,0}, t_{n}^{0}=0$ and $\lambda_{n}^{0}=1$. By (6.29) and (6.31), for $n$ large,

$$
\sum_{j=1}^{J}\left\|U_{n}^{j}(0)\right\|_{L^{2}}^{2}+\left\|w_{n}^{J}(0)\right\|_{L^{2}}^{2} \lesssim \delta\left(\alpha^{*}\right) \leq \frac{\delta_{0}^{2}}{2} .
$$

Define $U_{n}^{j}(\tau)$ and $w_{n}^{J}(\tau)$ the (global) solutions of the nonlinear equation (1.1) corresponding to the initial data $U_{n}^{j}(0)$ and $w_{n}^{J}(0)$. Let $\tau_{0}<0$ be such that $v(\tau)$ exists on $\left[\tau_{0}, 0\right]$. We claim that, for $n$ large, $v_{n}$ exists on $\left[\tau_{0}, 0\right]$ and

$$
\lim _{J \rightarrow+\infty} \limsup _{n \rightarrow+\infty} \sup _{\tau \in\left[\tau_{0}, 0\right]}\left\|v_{n}(\tau)-v(\tau)-\sum_{j=1}^{J} U_{n}^{j}(\tau)-w_{n}^{J}(\tau)\right\|_{L^{2}}=0 .
$$

Indeed, (6.33) is a by now standard corollary of the perturbation Lemma 2.4 (see e.g. Proposition 2.8 in [8]), In particular, there exist $n_{0}>1$ and $J_{0} \geq 1$ such that for $n>n_{0}$,

$$
\left\|v_{n}(\tau)-v(\tau)-\sum_{j=1}^{J_{0}} U_{n}^{j}(\tau)-w_{n}^{J_{0}}(\tau)\right\|_{L^{2}} \leq \delta_{0}
$$

and thus, for all $\tau \in\left[\tau_{0}, 0\right]$,

$$
\left\|v_{n}(\tau)-v(\tau)\right\|_{L^{2}} \leq\left\|\sum_{j=1}^{J_{0}} U_{n}^{j}(\tau)\right\|_{L^{2}}+\left\|w_{n}^{J_{0}}(\tau)\right\|_{L^{2}}+\delta_{0} \lesssim \delta_{0}<\frac{1}{10}\|Q\|_{L^{2}},
$$

choosing now $\delta_{0}$ small but universal. In particular, let $A$ be such that, for all $\tau \in\left[\tau_{0}, 0\right]$,

$$
\int_{|x|>A} v^{2}(\tau, x) d x<\frac{1}{100} \int Q^{2}
$$

and thus from (6.34):

$$
\int_{|x|>A} v_{n}^{2}(\tau, x) d x \leq \frac{1}{25} \int Q^{2}
$$


Now, recall from (6.15) that $x_{v_{n}}\left(\tau_{n}^{*}\right) \rightarrow-\infty$ as $n \rightarrow \infty$, and in particular, for $n$ large enough, $-x_{v_{n}}\left(\tau_{n}^{*}\right) \ll A$ and thus,

$$
\int_{x<-A} v_{n}^{2}\left(\tau_{n}^{*}, x\right) d x \geq \frac{3}{4} \int Q^{2} .
$$

We conclude that necessarily $\tau_{0}>\tau_{n}^{*}$ for $n$ large enough, and thus

$$
\tau_{0} \geq-\tau^{*}=\lim _{n} \tau_{n}^{*}
$$

It follows that $v(\tau)$ blows up in a finite time $\tau_{\max }(v) \geq-\tau^{*}=\lim _{n} \tau_{n}^{*}$. Since $\|v(0)\|_{L^{2}} \leq\|Q\|_{L^{2}}$ and $v(0) \in H^{1}$, we have $\|v\|_{L^{2}}=\|Q\|_{L^{2}}$. In particular, by weak convergence, and $\lim _{n \rightarrow \infty}\left\|v_{n}(0)\right\|_{L^{2}}=\|v(0)\|_{L^{2}}$, we obtain $\lim _{n \rightarrow \infty} \| v_{n}(0)-$ $v(0) \|_{L^{2}}=0$.

From the uniqueness statement in Theorem 1.3, there exists $\lambda^{*}>0, x^{*} \in \mathbb{R}$ and $\sigma^{*}>0$ such that

$$
v(0, x)=\left(\lambda^{*}\right)^{\frac{1}{2}} S\left(\sigma^{*}, \lambda^{*} x+x^{*}\right) .
$$

Moreover, denoting by $\left(b_{S}, \lambda_{S}, x_{S}\right)$ the parameters of the decomposition of $S$, we observe that

$$
\lambda_{v}(0)=1=\left(\lambda^{*}\right)^{-1} \lambda_{S}\left(\sigma^{*}\right), \quad x_{v}(0)=0=x_{S}\left(\sigma^{*}\right)-x^{*},
$$

and thus

$$
v(0, x)=\lambda_{S}^{\frac{1}{2}}\left(\sigma^{*}\right) S\left(\sigma^{*}, \lambda_{S}\left(\sigma^{*}\right) x+x_{S}\left(\sigma^{*}\right)\right) .
$$

In particular, by scaling

$$
v(\tau, x)=\lambda_{S}^{\frac{1}{2}}\left(\sigma^{*}\right) S\left(\sigma^{*}+\lambda_{S}^{3}\left(\sigma^{*}\right) \tau, \lambda_{S}\left(\sigma^{*}\right) x+x_{S}\left(\sigma^{*}\right)\right) .
$$

Since $v$ blows up at $\tau_{\max }(v)$, and $S$ blows up at time 0 (by convention), we have

$$
\sigma^{*}=-\lambda_{S}^{3}\left(\sigma^{*}\right) \tau_{\max }(v)<\lambda_{S}^{3}\left(\sigma^{*}\right) \tau_{*} .
$$

From the definition of $t_{n}^{*}$ and then strong $L^{2}$ convergence, we have

$$
\begin{aligned}
\alpha^{*} & =\inf _{\lambda_{1}, x_{1}}\left\|u_{n}\left(t_{n}^{*}\right)-Q_{\lambda_{1}}\left(.-x_{1}\right)\right\|_{L^{2}}=\inf _{\lambda_{1}, x_{1}}\left\|v_{n}(0)-Q_{\lambda_{1}}\left(.-x_{1}\right)\right\|_{L^{2}} \\
& =\inf _{\lambda_{1}, x_{1}}\left\|S\left(\sigma^{*}\right)-Q_{\lambda_{1}}\left(.-x_{1}\right)\right\|_{L^{2}} .
\end{aligned}
$$

Moreover, recall that by definition of $t_{n}^{*}$ and $\tau_{n}^{*}$, for all $\tau \in\left[\tau_{n}^{*}, 0\right]$, we have

$$
\inf _{\lambda_{1}, x_{1}}\left\|v_{n}(\tau)-Q_{\lambda_{1}}\left(.-x_{1}\right)\right\|_{L^{2}} \leq \alpha^{*},
$$

and so for all $t \in\left(0, \sigma^{*}\right]$,

$$
\inf _{\lambda_{1}, x_{1}}\left\|S(t)-Q_{\lambda_{1}}\left(.-x_{1}\right)\right\|_{L^{2}} \leq \alpha^{*} .
$$

From (4.35), we fix $t_{S}>0$ such that the distance of $S(t)$ to the family of solitons is increasing on $\left(0, t_{S}\right)$. Take $\alpha^{*}>0$ small enough so that

$$
\alpha^{*} \leq \frac{1}{2} \inf _{\lambda_{1}, x_{1}}\left\|S\left(t_{S}\right)-Q_{\lambda_{1}}\left(.-x_{1}\right)\right\|_{L^{2}}
$$

By (6.36), it is clear that $\sigma^{*} \in\left(0, t_{S}\right)$. Moreover, $\sigma^{*}$ is uniquely defined by (6.35) (small for $\alpha^{*}$ small) and thus does not depend on the subsequence but only on $\alpha^{*}$. In particular, all the sequence converges to the same limit and the proposition is proved. 


\section{Appendix A. End of the proof of Proposition 4.1}

In this appendix, we finish the proof of Proposition 4.1 by proving (4.11) and (4.12)-(4.13) in the framework of Proposition 4.1. For the reader's convenience, we recall the main estimates proved at this point on $\varepsilon$ and the parameters $b, \lambda, x$ : for $|s|$ large,

$$
\begin{gathered}
\|\varepsilon(s)\|_{L^{\infty}} \lesssim\|\varepsilon(s)\|_{H^{1}} \lesssim \frac{1}{\sqrt{|s|}}, \quad \frac{c_{1}\left(u_{0}\right)}{\sqrt{|s|}} \leq \lambda(s) \leq \frac{c_{2}\left(u_{0}\right)}{\sqrt{|s|}}, \quad b(s) \sim \frac{1}{2 s}, \\
\mathcal{N}(s)+\int_{-\infty}^{s} \int\left(\varepsilon_{y}^{2}+\varepsilon^{2}\right)\left(s^{\prime}\right) \varphi_{B}^{\prime} d s^{\prime} \lesssim \frac{1}{|s|^{3}}, \\
\left|\frac{\lambda_{s}}{\lambda}+b\right|+\left|\frac{x_{s}}{\lambda}-1\right| \lesssim \frac{1}{|s|^{\frac{3}{2}}}, \quad\left|b_{s}\right| \lesssim \frac{1}{|s|^{2}} .
\end{gathered}
$$

A.1. Proof of (4.11). Since $u(t)$ is a minimal mass blowing up solution and $\lambda(s)$ is increasing for $|s|$ large, from Lemma 2.11 and then using the properties of $Q_{b}$ (see Lemma 2.5), we obtain for $|s|$ large,

$$
\forall y>0,|\varepsilon(s, y)| \lesssim e^{-\frac{y}{20}}
$$

Thus, by (A.1),

$$
\lim _{s \rightarrow-\infty} \int \varepsilon^{2}(s, y) e^{\lambda(s) y} d y=0 .
$$

Now, to prove (4.11), we compute the time derivative of $\int \varepsilon^{2} e^{\lambda y}$. Using the equation of $\varepsilon$ (see (2.24) $)$, we have

$$
\begin{aligned}
\frac{1}{2} \frac{d}{d s} \int \varepsilon^{2} e^{\lambda y} & =\frac{\lambda_{s}}{2} \int y e^{\lambda y} \varepsilon^{2}+\int \varepsilon_{s} \varepsilon e^{\lambda y} \\
& =\frac{\lambda_{s}}{2} \int y e^{\lambda y} \varepsilon^{2}+\int(L \varepsilon)_{y} \varepsilon e^{\lambda y}+\left(\frac{\lambda_{s}}{\lambda}+b\right) \int \Lambda Q_{b} \varepsilon e^{\lambda y}+\frac{\lambda_{s}}{\lambda} \int \varepsilon \Lambda \varepsilon e^{\lambda y} \\
& +\left(\frac{x_{s}}{\lambda}-1\right) \int\left(Q_{b}+\varepsilon\right)_{y} \varepsilon e^{\lambda y}+\int \Phi_{b} \varepsilon e^{\lambda y}+\int \Psi_{b} \varepsilon e^{\lambda y} \\
& -\int\left(R_{b}(\varepsilon)\right)_{y} \varepsilon e^{\lambda y}-\int\left(R_{N L}(\varepsilon)\right)_{y} \varepsilon e^{\lambda y}
\end{aligned}
$$

Since $\int \varepsilon \Lambda \varepsilon e^{\lambda y}=-\frac{1}{2} \lambda \int \varepsilon^{2} y e^{\lambda y}$, the scaling terms cancel (this is because the quantity is scaling invariant). Next, using (A.3), we have:

$$
\begin{aligned}
& \int(L \varepsilon)_{y} \varepsilon e^{\lambda y}=-\frac{3}{2} \lambda \int \varepsilon_{y}^{2} e^{\lambda y}-\frac{1}{2} \lambda\left(1-\lambda^{2}\right) \int \varepsilon^{2} e^{\lambda y}+\int\left(-10 Q^{3} Q^{\prime}-\frac{5}{2} \lambda Q^{4}\right) \varepsilon^{2} e^{\lambda y} \\
& \leq-\frac{\lambda}{4} \int\left(\varepsilon_{y}^{2}+\varepsilon^{2}\right) e^{\lambda y}+C \mathcal{N}_{\mathrm{loc}}, \\
&\left|\left(\frac{\lambda_{s}}{\lambda}+b\right) \int \Lambda Q_{b} \varepsilon e^{\lambda y}\right|+\left|\left(\frac{x_{s}}{\lambda}-1\right) \int\left(Q_{b}\right)_{y} \varepsilon e^{\lambda y}\right| \\
& \lesssim \frac{1}{|s|^{\frac{3}{2}}}\left[\left(\int \varepsilon^{2} e^{-\frac{|y|}{10}}\right)^{\frac{1}{2}}+|b|\left(\int \varepsilon^{2} e^{\lambda y}\right)^{\frac{1}{2}}\left(\iint_{y<0} e^{\lambda y}\right)^{\frac{1}{2}}\right] \\
& \lesssim \frac{1}{|s|^{\frac{3}{2}}}\left[\frac{1}{|s|^{\frac{3}{2}}}+\frac{1}{\sqrt{|s|}}\left(\int \varepsilon^{2} e^{\lambda y}\right)^{\frac{1}{2}}\right] \lesssim \frac{\lambda}{100} \int \varepsilon^{2} e^{\lambda y}+\frac{1}{|s|^{3}}, \\
&\left|\left(\frac{x_{s}}{\lambda}-1\right) \int \varepsilon_{y} \varepsilon e^{\lambda y}\right|=\frac{\lambda}{2}\left|\left(\frac{x_{s}}{\lambda}-1\right) \int \varepsilon^{2} e^{\lambda y}\right| \leq \frac{\lambda}{100} \int \varepsilon^{2} e^{\lambda y} .
\end{aligned}
$$




$$
\begin{aligned}
\left|\int \Phi_{b} \varepsilon e^{\lambda y}\right| & \lesssim\left|b_{s}\right| \int|P||\varepsilon| e^{\lambda y} \lesssim \frac{1}{|s|^{2}}\left(\int P^{2} e^{\lambda y}\right)^{\frac{1}{2}}\left(\int \varepsilon^{2} e^{\lambda y}\right)^{\frac{1}{2}} \\
& \lesssim \frac{1}{|s|^{\frac{7}{4}}}\left(\int \varepsilon^{2} e^{\lambda y}\right)^{\frac{1}{2}} \leq \frac{\lambda}{100} \int \varepsilon^{2} e^{\lambda y}+\frac{C}{|s|^{3}} .
\end{aligned}
$$

Using (2.14) (recall $\left.\gamma=\frac{3}{4}\right)$,

$$
\begin{aligned}
& \left|\int \Psi_{b} \varepsilon e^{\lambda y}\right| \lesssim|b|^{\frac{7}{4}} \int_{-2|b|^{-\frac{3}{4}}<y<-|b|^{-\frac{3}{4}}}|\varepsilon| e^{\lambda y}+|b|^{2} \int_{y<0}|\varepsilon| e^{\lambda y}+|b|^{2} \int_{y>0}|\varepsilon| e^{-\frac{y}{4}} \\
& \lesssim \frac{1}{|s|^{\frac{7}{4}}}\left(\int_{y<-|b|^{-\frac{3}{4}}} e^{\lambda y}\right)^{\frac{1}{2}}\left(\int \varepsilon^{2} e^{\lambda y}\right)^{\frac{1}{2}}+\frac{1}{|s|^{2}}\left(\int_{y<0} e^{\lambda y}\right)^{\frac{1}{2}}\left(\int \varepsilon^{2} e^{\lambda y}\right)^{\frac{1}{2}}+\frac{1}{|s|^{3}} \\
& \lesssim\left(\frac{1}{|s|^{\frac{3}{2}}} e^{-\lambda|b|^{-\frac{3}{4}}}+\frac{1}{|s|^{\frac{7}{4}}}\right)\left(\int \varepsilon^{2} e^{\lambda y}\right)^{\frac{1}{2}}+\frac{1}{|s|^{3}} \leq \frac{\lambda}{100} \int \varepsilon^{2} e^{\lambda y}+\frac{C}{|s|^{3}} .
\end{aligned}
$$

Next, since $\left|R_{b}(\varepsilon)\right|=5\left|Q_{b}^{4}-Q^{4}\right||\varepsilon| \lesssim|b||\varepsilon|$, we have

$$
\begin{aligned}
\left|\int\left(R_{b}(\varepsilon)\right)_{y} \varepsilon e^{\lambda y}\right| & \leq\left|\int R_{b}(\varepsilon)\left(\lambda|\varepsilon|+\left|\varepsilon_{y}\right|\right) e^{\lambda y}\right| \\
& \lesssim|b| \int\left(\varepsilon_{y}^{2}+\varepsilon^{2}\right) e^{\lambda y} \leq \frac{\lambda}{100} \int\left(\varepsilon_{y}^{2}+\varepsilon^{2}\right) e^{\lambda y}
\end{aligned}
$$

Finally, since

$$
\left|R_{\mathrm{NL}}(\varepsilon)\right| \leq\|\varepsilon\|_{L^{\infty}}\left(\|\varepsilon\|_{L^{\infty}}^{3}+|b|+e^{-\frac{y}{10}}\right)|\varepsilon|
$$

we get

$$
\begin{aligned}
\left|\int\left(R_{\mathrm{NL}}(\varepsilon)\right)_{y} \varepsilon e^{\lambda y}\right| & \leq\left|\int R_{\mathrm{NL}}(\varepsilon)\left(\lambda|\varepsilon|+\left|\varepsilon_{y}\right|\right) e^{\lambda y}\right| \\
& \lesssim \frac{1}{|s|^{\frac{3}{2}}} \int\left(\varepsilon_{y}^{2}+\varepsilon^{2}\right) e^{\lambda y} \leq \frac{\lambda}{100} \int\left(\varepsilon_{y}^{2}+\varepsilon^{2}\right) e^{\lambda y} .
\end{aligned}
$$

The collection of above bound ensures

$$
\frac{d}{d s} \int \varepsilon^{2} e^{\lambda y} \lesssim \frac{1}{|s|^{3}}
$$

which integration on $(-\infty, s]$ using (A.5) yields (4.11).

A.2. Proofs of (4.12)-(4.13). Note first that by standard arguments,

$$
\left\|\left(\left(\partial_{y}^{2} \varepsilon\right)^{2}+\left(\partial_{y} \varepsilon\right)^{2}\right)(s) e^{\omega y}\right\|_{L^{\infty}} \lesssim \int\left(\left(\partial_{y}^{3} \varepsilon\right)^{2}+\left(\partial_{y}^{2} \varepsilon\right)^{2}+\left(\partial_{y} \varepsilon\right)^{2}+\varepsilon^{2}\right)(s, y) e^{\omega y} d y,
$$

and so it is sufficient to prove (4.12).

The proof is similar to Section 3.4 in [25] and involves some computations originally introduced in [13]. To prove (4.12), we need only rough bounds on $\varepsilon$ and it is therefore simpler to decompose

$$
\varepsilon+Q_{b}=\tilde{\varepsilon}+Q
$$

which satisfies:

$$
\partial_{s} \tilde{\varepsilon}+\partial_{y}\left(\partial_{y}^{2} \tilde{\varepsilon}-\tilde{\varepsilon}+F(\tilde{\varepsilon})\right)=\frac{\lambda_{s}}{\lambda}(\Lambda Q+\Lambda \tilde{\varepsilon})+\left(\frac{x_{s}}{\lambda}-1\right)\left(\partial_{y} Q+\partial_{y} \tilde{\varepsilon}\right),
$$

with

$$
F(\tilde{\varepsilon})=(Q+\tilde{\varepsilon})^{5}-Q^{5}
$$


From (A.1), (A.2) and $Q_{b}-Q=b P \chi_{b}$ (see Lemma 2.5) we have the following estimates on $\tilde{\varepsilon}$ :

$$
\|\tilde{\varepsilon}\|_{L^{\infty}} \lesssim \frac{1}{\sqrt{|s|}}, \quad \int \tilde{\varepsilon}^{2} e^{-\frac{|y|}{10}} \leq \frac{1}{|s|^{2}}
$$

From (A.2),

$$
\int \varepsilon^{2}(s) \varphi_{B}+\int_{-\infty}^{s} \int\left(\varepsilon_{y}^{2}+\varepsilon^{2}\right)\left(s^{\prime}\right) \varphi_{B} d s^{\prime} \lesssim \frac{1}{|s|^{3}}
$$

and thus, since $|b(s)| \lesssim \frac{1}{|s|}$, for $|s|$ large,

$$
\int \tilde{\varepsilon}^{2}(s) \varphi_{B}+\int_{-\infty}^{s} \int\left(\tilde{\varepsilon}_{y}^{2}+\tilde{\varepsilon}^{2}\right)\left(s^{\prime}\right) \varphi_{B} d s^{\prime} \lesssim \frac{1}{|s|} .
$$

Moreover, since $u(t)$ is a minimal mass blowing up solution and $\lambda(s)$ is increasing for $|s|$ large, from Lemma 2.11 and then using the properties of $Q_{b}$ (see Lemma 2.5), we obtain for $|s|$ large,

$$
\forall y>0,|\varepsilon(s, y)| \lesssim e^{-\frac{y}{20}} \quad \text { and so } \quad \forall y>0,|\tilde{\varepsilon}(s, y)| \lesssim e^{-\frac{y}{20}}
$$

In particular, it follows that for all $\frac{1}{B} \leq \omega<\frac{1}{10}$,

$$
\lim _{s \rightarrow-\infty} \int \tilde{\varepsilon}^{2}(s, y) e^{\omega y} d y=0 .
$$

step 1 We claim that for all $\frac{1}{B}<\omega<\frac{1}{10}$, for $|s|$ large,

$$
\int \tilde{\varepsilon}^{2}(s, y) e^{\omega y} d y+\int_{-\infty}^{s} \int\left(\tilde{\varepsilon}_{y}^{2}\left(s^{\prime}, y\right)+\tilde{\varepsilon}^{2}\left(s^{\prime}, y\right)\right) e^{\omega y} d y d s^{\prime} \lesssim \frac{1}{|s|} .
$$

Define

$$
H_{0}(s)=\frac{1}{2} \int \tilde{\varepsilon}^{2}(s, y) e^{\omega y} d y
$$

Then,

$$
\begin{aligned}
& \frac{d}{d s} H_{0}=\int \tilde{\varepsilon}_{s} \tilde{\varepsilon} e^{\omega y}=-\int\left(-\tilde{\varepsilon}_{y y}+\tilde{\varepsilon}-F(\tilde{\varepsilon})\right)\left(\tilde{\varepsilon} e^{\omega y}\right)_{y}+\frac{\lambda_{s}}{\lambda} \int(\Lambda Q+\Lambda \tilde{\varepsilon}) \tilde{\varepsilon} e^{\omega y} \\
& +\left(\frac{x_{s}}{\lambda}-1\right) \int\left(Q^{\prime}+\tilde{\varepsilon}_{y}\right) \tilde{\varepsilon} e^{\omega y} \\
& =-\frac{3}{2} \omega \int \tilde{\varepsilon}_{y}^{2} e^{\omega y}-\frac{1}{2} \omega\left(1-\omega^{2}\right) \int \tilde{\varepsilon}^{2} e^{\omega y}+\int F(\tilde{\varepsilon})\left(\tilde{\varepsilon} e^{\omega y}\right)_{y} \\
& +\frac{\lambda_{s}}{\lambda} \int \Lambda Q \tilde{\varepsilon} e^{\omega y}-\frac{\omega}{2} \frac{\lambda_{s}}{\lambda} \int \tilde{\varepsilon}^{2} y e^{\omega y}+\left(\frac{x_{s}}{\lambda}-1\right) \int Q^{\prime} \tilde{\varepsilon} e^{\omega y}-\frac{\omega}{2}\left(\frac{x_{s}}{\lambda}-1\right) \int \tilde{\varepsilon}^{2} e^{\omega y} .
\end{aligned}
$$

First, by decay properties of $Q$ and since $\|\tilde{\varepsilon}\|_{L^{\infty}}^{2} \lesssim \frac{1}{|s|}$ (by (A.7)), for $|s|$ large,

$$
\begin{aligned}
\left|\int F(\tilde{\varepsilon})\left(\tilde{\varepsilon} e^{\omega y}\right)_{y}\right| & \lesssim \int\left(|\tilde{\varepsilon}| Q^{4}+|\tilde{\varepsilon}|^{5}\right)\left(\left|\tilde{\varepsilon}_{y}\right|+|\tilde{\varepsilon}|\right) e^{\omega y} \\
& \lesssim \frac{\omega}{100} \int\left(\tilde{\varepsilon}_{y}^{2}+\tilde{\varepsilon}^{2}\right) e^{\omega y}+\int\left(\tilde{\varepsilon}_{y}^{2}+\tilde{\varepsilon}^{2}\right) \varphi_{B} .
\end{aligned}
$$

Second, by $\left|\frac{\lambda_{s}}{\lambda}\right|+\left|\frac{x_{s}}{\lambda}-1\right| \lesssim \frac{1}{|s|}$, the decay properties of $Q$ and (A.7)

$$
\left|\frac{\lambda_{s}}{\lambda} \int \Lambda Q \tilde{\varepsilon} e^{\omega y}\right|+\left|\left(\frac{x_{s}}{\lambda}-1\right) \int Q^{\prime} \tilde{\varepsilon} e^{\omega y}\right| \lesssim \frac{1}{|s|}\left(\int \tilde{\varepsilon}^{2} e^{-\frac{|y|}{10}}\right)^{\frac{1}{2}} \lesssim \frac{1}{|s|^{2}} .
$$


52

Y. MARTEL, F. MERLE, AND P. RAPHAËL

Finally, for $\frac{1}{B}<\omega^{\prime \prime}<\omega<\omega^{\prime} \leq \frac{1}{10}$, and then using (A.10),

$$
\begin{aligned}
\left|\frac{\lambda_{s}}{\lambda} \int \tilde{\varepsilon}^{2} y e^{\omega y}\right| & \lesssim \frac{1}{|s|}\left(\int \tilde{\varepsilon}^{2} y^{2} e^{\omega y}\right)^{\frac{1}{2}}\left(\int \tilde{\varepsilon}^{2} e^{\omega y}\right)^{\frac{1}{2}} \\
& \lesssim \frac{1}{|s|}\left(\int \tilde{\varepsilon}^{2}\left(e^{\omega^{\prime \prime} y}+e^{\omega^{\prime} y}\right)\right)^{\frac{1}{2}}\left(\int \tilde{\varepsilon}^{2} e^{\omega y}\right)^{\frac{1}{2}} \lesssim \frac{\omega}{100} \int \tilde{\varepsilon}^{2} e^{\omega y}+\frac{1}{s^{2}} .
\end{aligned}
$$

In conclusion, we get

$$
\frac{d}{d s} H_{0} \leq-\frac{\omega}{4} \int\left(\tilde{\varepsilon}_{y}^{2}+\tilde{\varepsilon}^{2}\right) e^{\omega y} d y+C \int\left(\tilde{\varepsilon}_{y}^{2}+\tilde{\varepsilon}^{2}\right) \varphi_{B}+\frac{C}{|s|^{2}} .
$$

Integrating on $(-\infty, s]$, using (A.8) and $\lim _{s \rightarrow-\infty} H_{0}(s)=0$ by (A.10), we get (A.11). In particular, for some sequence $s_{n} \rightarrow-\infty$,

$$
\lim _{n \rightarrow \infty} \int \tilde{\varepsilon}_{y}^{2}\left(s_{n}, y\right) e^{\omega y} d y=0 .
$$

step 2 We claim that for all $\frac{1}{B}<\omega<\frac{1}{10}$, for $|s|$ large,

$$
\int \tilde{\varepsilon}_{y}^{2}(s, y) e^{\omega y} d y+\int_{-\infty}^{s} \int \tilde{\varepsilon}_{y y}^{2}\left(s^{\prime}, y\right) e^{\omega y} d y d s^{\prime} \lesssim \frac{1}{|s|} .
$$

Define

$$
H_{1}(s)=\int \frac{1}{2}\left(\tilde{\varepsilon}_{y}^{2}(s, y)+\tilde{\varepsilon}^{2}(s, y)\right) e^{\omega y}-\left(\frac{(Q+\tilde{\varepsilon})^{6}}{6}-\tilde{\varepsilon} Q^{5}-\frac{Q^{6}}{6}\right) e^{\omega y} d y .
$$

Then,

$$
\begin{aligned}
& \frac{d}{d s} H_{1}=\int \tilde{\varepsilon}_{s}\left(-\tilde{\varepsilon}_{y y}+\tilde{\varepsilon}-F(\tilde{\varepsilon})\right) e^{\omega y}-\omega \int \tilde{\varepsilon}_{s} \tilde{\varepsilon}_{y} e^{\omega y} \\
& =-\frac{\omega}{2} \int\left(-\tilde{\varepsilon}_{y y}+\tilde{\varepsilon}-F(\tilde{\varepsilon})\right)^{2} e^{\omega y}-\omega \int\left(-\tilde{\varepsilon}_{y y}+\tilde{\varepsilon}-F(\tilde{\varepsilon})\right)_{y} \tilde{\varepsilon}_{y} e^{\omega y} \\
& +\frac{\lambda_{s}}{\lambda} \int(\Lambda Q+\Lambda \tilde{\varepsilon})\left(-\tilde{\varepsilon}_{y y}+\tilde{\varepsilon}-F(\tilde{\varepsilon})\right) e^{\omega y}+\omega \frac{\lambda_{s}}{\lambda} \int(\Lambda Q+\Lambda \tilde{\varepsilon}) \tilde{\varepsilon}_{y} e^{\omega y} \\
& +\left(\frac{x_{s}}{\lambda}-1\right) \int\left(Q^{\prime}+\tilde{\varepsilon}_{y}\right)\left(-\tilde{\varepsilon}_{y y}+\tilde{\varepsilon}-F(\tilde{\varepsilon})\right) e^{\omega y}+\omega\left(\frac{x_{s}}{\lambda}-1\right) \int\left(Q^{\prime}+\tilde{\varepsilon}_{y}\right) \tilde{\varepsilon}_{y} e^{\omega y} \\
& \leq-\omega \int \tilde{\varepsilon}_{y y}^{2} e^{\omega y}-\omega\left(1-\frac{1}{2} \omega^{2}\right) \int \tilde{\varepsilon}_{y}^{2} e^{\omega y}-\omega \int F(\tilde{\varepsilon})\left(\tilde{\varepsilon}_{y} e^{\omega y}\right)_{y} \\
& +\frac{\lambda_{s}}{\lambda} \int(\Lambda Q+\Lambda \tilde{\varepsilon})\left(-\tilde{\varepsilon}_{y y}+\tilde{\varepsilon}-F(\tilde{\varepsilon})\right) e^{\omega y}+\omega \frac{\lambda_{s}}{\lambda} \int(\Lambda Q+\Lambda \tilde{\varepsilon}) \tilde{\varepsilon}_{y} e^{\omega y} \\
& +\left(\frac{x_{s}}{\lambda}-1\right) \int\left(Q^{\prime}+\tilde{\varepsilon}_{y}\right)\left(-\tilde{\varepsilon}_{y y}+\tilde{\varepsilon}-F(\tilde{\varepsilon})\right) e^{\omega y}+\omega\left(\frac{x_{s}}{\lambda}-1\right) \int\left(Q^{\prime}+\tilde{\varepsilon}_{y}\right) \tilde{\varepsilon}_{y} e^{\omega y}
\end{aligned}
$$

First, as in step 1, for $|s|$ large,

$$
\begin{aligned}
\left|\int F(\tilde{\varepsilon})\left(\tilde{\varepsilon}_{y} e^{\omega y}\right)_{y}\right| & \lesssim \int\left(|\tilde{\varepsilon}| Q^{4}+|\tilde{\varepsilon}|^{5}\right)\left(\left|\tilde{\varepsilon}_{y y}\right|+\left|\tilde{\varepsilon}_{y}\right|\right) e^{\omega y} \\
& \lesssim \frac{\omega}{100} \int\left(\tilde{\varepsilon}_{y y}^{2}+\tilde{\varepsilon}_{y}^{2}+\tilde{\varepsilon}^{2}\right) e^{\omega y}+\int\left(\tilde{\varepsilon}_{y}^{2}+\tilde{\varepsilon}^{2}\right) \varphi_{B} .
\end{aligned}
$$


Second, the following estimates are proved as in step 1, (A.12), after possible integrations by parts

$$
\begin{aligned}
& \left|\frac{\lambda_{s}}{\lambda} \int \Lambda Q\left(-\tilde{\varepsilon}_{y y}+\tilde{\varepsilon}-F(\tilde{\varepsilon})\right) e^{\omega y}\right|+\left|\frac{\lambda_{s}}{\lambda} \int \Lambda Q \tilde{\varepsilon}_{y} e^{\omega y}\right| \\
& +\left|\left(\frac{x_{s}}{\lambda}-1\right) \int Q^{\prime}\left(-\tilde{\varepsilon}_{y y}+\tilde{\varepsilon}-F(\tilde{\varepsilon})\right) e^{\omega y}\right|+\left|\left(\frac{x_{s}}{\lambda}-1\right) \int Q^{\prime} \tilde{\varepsilon}_{y} e^{\omega y}\right| \lesssim \frac{1}{s^{2}} .
\end{aligned}
$$

For example, by the decay properties of $Q$ and (A.8),

$$
\left|\frac{\lambda_{s}}{\lambda} \int \Lambda Q \tilde{\varepsilon}_{y y} e^{\omega y}\right| \lesssim \frac{1}{|s|} \int\left|\left(\Lambda Q e^{\omega y}\right)_{y y}\right||\tilde{\varepsilon}| \lesssim \frac{1}{s^{2}} .
$$

Finally, we observe that

$$
\int(\Lambda \tilde{\varepsilon}) \tilde{\varepsilon}_{y y} e^{\omega y}=\int\left(-\tilde{\varepsilon}_{y}^{2} e^{\omega y}+\frac{\omega^{2}}{2} \tilde{\varepsilon}^{2} e^{\omega y}+\tilde{\varepsilon}_{y}^{2}\left(y e^{\omega y}\right)_{y}\right),
$$

and thus for some $\frac{1}{B}<\omega^{\prime \prime}<\omega<\omega^{\prime}<\frac{1}{10}$,

$$
\left|\frac{\lambda_{s}}{\lambda} \int(\Lambda \tilde{\varepsilon}) \tilde{\varepsilon}_{y y} e^{\omega y}\right| \lesssim \frac{1}{|s|} \int\left(\tilde{\varepsilon}_{y}^{2}+\tilde{\varepsilon}^{2}\right)\left(e^{\omega^{\prime} y}+e^{\omega^{\prime \prime} y}\right) .
$$

All the remaining terms are easier and are treated similarly as in step 1.

The collection of above bounds yields:

$$
\begin{aligned}
\frac{d}{d s} H_{1} & \lesssim-\int\left(\tilde{\varepsilon}_{y y}^{2}+\tilde{\varepsilon}_{y}^{2}+\tilde{\varepsilon}^{2}\right) e^{\omega y} d y+\int\left(\tilde{\varepsilon}_{y}^{2}+\tilde{\varepsilon}^{2}\right) \varphi_{B} \\
& +\int\left(\tilde{\varepsilon}_{y}^{2}+\tilde{\varepsilon}^{2}\right)\left(e^{\omega^{\prime} y}+e^{\omega^{\prime \prime} y}\right)+\frac{1}{|s|^{2}}
\end{aligned}
$$

Note that $\lim _{n \rightarrow \infty} H_{1}\left(s_{n}\right)=0$ by (A.10) and (A.13). Integrating on $\left[s_{n}, s\right]$, and then passing to the limit as $n \rightarrow+\infty$, using (A.8) and (A.11) for $\omega^{\prime}$ and $\omega^{\prime \prime}$, we find (A.14). In particular, there exists a subsequence still denoted $\left(s_{n}\right)$ such that

$$
\lim _{n \rightarrow \infty} \int\left(\tilde{\varepsilon}_{y y}^{2}+\varepsilon_{y}^{2}+\tilde{\varepsilon}^{2}\right)\left(s_{n}, y\right) e^{\omega y} d y=0 .
$$

step 3 We claim that for all $\frac{3}{B}<\omega<\frac{1}{10}$, for $|s|$ large,

$$
\int \tilde{\varepsilon}_{y y}^{2}(s, y) e^{\omega y} d y+\int_{-\infty}^{s} \int \tilde{\varepsilon}_{y y y}^{2}\left(s^{\prime}, y\right) e^{\omega y} d y d s^{\prime} \lesssim \frac{1}{|s|} .
$$

Define

$$
H_{2}(s)=\frac{1}{2} \int \tilde{\varepsilon}_{y y}^{2} e^{\omega y}-\frac{25}{6} \int \tilde{\varepsilon}_{y}^{2} \tilde{\varepsilon}^{4} e^{\omega y}
$$

Then,

$$
\frac{d}{d s} H_{2}=\int \tilde{\varepsilon}_{y y s} \tilde{\varepsilon}_{y y} e^{\omega y}-\frac{25}{3} \int\left(\tilde{\varepsilon}_{y s} \tilde{\varepsilon}_{y} \tilde{\varepsilon}^{4}+2 \tilde{\varepsilon}_{s} \tilde{\varepsilon}_{y}^{2} \tilde{\varepsilon}^{3}\right) e^{\omega y}=H_{2,1}+H_{2,2}
$$


First,

$$
\begin{aligned}
H_{2,1} & =\int\left(-\tilde{\varepsilon}_{y y}+\tilde{\varepsilon}-F(\tilde{\varepsilon})\right)_{y y y} \varepsilon_{y y} e^{\omega y} \\
& +\frac{\lambda_{s}}{\lambda} \int(\Lambda Q+\Lambda \tilde{\varepsilon})_{y y} \tilde{\varepsilon}_{y y} e^{\omega y}+\left(\frac{x_{s}}{\lambda}-1\right) \int\left(Q^{\prime}+\tilde{\varepsilon}_{y}\right)_{y y} \tilde{\varepsilon}_{y y} e^{\omega y} \\
& =-\frac{3}{2} \omega \int \tilde{\varepsilon}_{y y y}^{2} e^{\omega y}-\frac{\omega}{2}\left(1-\omega^{2}\right) \int \tilde{\varepsilon}_{y y}^{2} e^{\omega y}-50 \int \tilde{\varepsilon}_{y y}^{2} \tilde{\varepsilon}_{y} \tilde{\varepsilon}^{3} e^{\omega y} \\
& +\int\left(F(\tilde{\varepsilon})-\tilde{\varepsilon}^{5}\right)_{y y}\left(\varepsilon_{y y} e^{\omega y}\right)_{y} \\
& +\frac{5}{2} \omega \int \tilde{\varepsilon}_{y y}^{2} \tilde{\varepsilon}^{4} e^{\omega y}+30 \int \tilde{\varepsilon}_{y}^{5} \tilde{\varepsilon} e^{\omega y}+15 \omega \int \tilde{\varepsilon}_{y}^{4} \tilde{\varepsilon}^{2} e^{\omega y} \\
& +\frac{\lambda_{s}}{\lambda} \int(\Lambda Q+\Lambda \tilde{\varepsilon})_{y y} \tilde{\varepsilon}_{y y} e^{\omega y}+\left(\frac{x_{s}}{\lambda}-1\right) \int\left(Q^{\prime}+\tilde{\varepsilon}_{y}\right)_{y y} \tilde{\varepsilon}_{y y} e^{\omega y} .
\end{aligned}
$$

Second,

$$
\begin{aligned}
H_{2,2} & =\frac{25}{3} \int \tilde{\varepsilon}_{s}\left(\left(\tilde{\varepsilon}_{y} \tilde{\varepsilon}^{4} e^{\omega y}\right)_{y}-2 \tilde{\varepsilon}_{y}^{2} \tilde{\varepsilon}^{3} e^{\omega y}\right) \\
& =\frac{25}{3} \int\left(-\tilde{\varepsilon}_{y y}+\tilde{\varepsilon}-F(\tilde{\varepsilon})\right)_{y}\left(\tilde{\varepsilon}_{y y} \tilde{\varepsilon}^{4}+2 \tilde{\varepsilon}_{y}^{2} \tilde{\varepsilon}^{3}+\omega \tilde{\varepsilon}_{y} \tilde{\varepsilon}^{4}\right) e^{\omega y} \\
& +\frac{25}{3} \frac{\lambda_{s}}{\lambda} \int(\Lambda Q+\Lambda \tilde{\varepsilon})\left(\tilde{\varepsilon}_{y y} \tilde{\varepsilon}^{4}+2 \tilde{\varepsilon}_{y}^{2} \tilde{\varepsilon}^{3}+\omega \tilde{\varepsilon}_{y} \tilde{\varepsilon}^{4}\right) e^{\omega y} \\
& +\frac{25}{3}\left(\frac{x_{s}}{\lambda}-1\right) \int\left(Q^{\prime}+\tilde{\varepsilon}_{y}\right)\left(\tilde{\varepsilon}_{y y} \tilde{\varepsilon}^{4}+2 \tilde{\varepsilon}_{y}^{2} \tilde{\varepsilon}^{3}+\omega \tilde{\varepsilon}_{y} \tilde{\varepsilon}^{4}\right) e^{\omega y} \\
& =50 \int \tilde{\varepsilon}_{y y}^{2} \tilde{\varepsilon}_{y} \tilde{\varepsilon}^{3} e^{\omega y}+\frac{25}{3} \omega \int \tilde{\varepsilon}_{y y}^{2} \tilde{\varepsilon}^{4} e^{\omega y}-\frac{25}{2} \int \tilde{\varepsilon}_{y}^{5} \tilde{\varepsilon}^{\omega y}-\frac{175}{12} \omega \int \tilde{\varepsilon}_{y}^{4} \tilde{\varepsilon}^{2} e^{\omega y} \\
& -\frac{25}{9} \omega^{3} \int \tilde{\varepsilon}_{y}^{3} \tilde{\varepsilon}^{3} e^{\omega y}-\frac{25}{3} \int\left(F(\tilde{\varepsilon})-\tilde{\varepsilon}^{5}\right)_{y}\left(\tilde{\varepsilon}_{y y} \tilde{\varepsilon}^{4}+2 \tilde{\varepsilon}_{y}^{2} \tilde{\varepsilon}^{3}+\omega \tilde{\varepsilon}_{y} \tilde{\varepsilon}^{4}\right) e^{\omega y} \\
& +\frac{25}{3} \frac{\lambda_{s}}{\lambda} \int(\Lambda Q+\Lambda \tilde{\varepsilon})\left(\tilde{\varepsilon}_{y y} \tilde{\varepsilon}^{4}+2 \tilde{\varepsilon}_{y}^{2} \tilde{\varepsilon}^{3}+\omega \tilde{\varepsilon}_{y} \tilde{\varepsilon}^{4}\right) e^{\omega y} \\
& +\frac{25}{3}\left(\frac{x_{s}}{\lambda}-1\right) \int\left(Q^{\prime}+\tilde{\varepsilon}_{y}\right)\left(\tilde{\varepsilon}_{y y} \tilde{\varepsilon}^{4}+2 \tilde{\varepsilon}_{y}^{2} \tilde{\varepsilon}^{3}+\omega \tilde{\varepsilon}_{y} \tilde{\varepsilon}^{4}\right) e^{\omega y} .
\end{aligned}
$$

The main observation when looking at the above expressions of $H_{2,1}$ and $H_{2,2}$ is that the higher order nonlinear term $\int \tilde{\varepsilon}_{y y}^{2} \tilde{\varepsilon}_{y} \tilde{\varepsilon}^{3} e^{\omega y}$ cancels in the expression of $\frac{d}{d s} H_{2}$. All other terms are now controlled as follows.

First, by (A.7),

$$
\left|\int \tilde{\varepsilon}_{y y}^{2} \tilde{\varepsilon}^{4} e^{\omega y}\right| \lesssim \frac{1}{|s|^{2}} \int \tilde{\varepsilon}_{y y}^{2} e^{\omega y}
$$

Second, by Holder inequality, (1.3), and then (A.11), (A.14), for $\frac{3}{100}<\omega<\frac{1}{10}$,

$$
\begin{aligned}
\left|\int \tilde{\varepsilon}_{y}^{5} \tilde{\varepsilon} e^{\omega y}\right| & \lesssim\left(\int \tilde{\varepsilon}_{y}^{6} e^{\omega y}\right)^{\frac{5}{6}}\left(\int \tilde{\varepsilon}^{6} e^{\omega y}\right)^{\frac{1}{6}} \\
& \lesssim\left(\int\left(\tilde{\varepsilon}_{y y}^{2}+\tilde{\varepsilon}_{y}^{2}+\tilde{\varepsilon}^{2}\right) e^{\frac{\omega}{3} y}\right)^{\frac{5}{6}}\left(\int\left(\tilde{\varepsilon}_{y}^{2}+\tilde{\varepsilon}^{2}\right) e^{\frac{\omega}{3} y}\right)^{\frac{13}{6}} \\
& \lesssim \int\left(\tilde{\varepsilon}_{y y}^{2}+\tilde{\varepsilon}_{y}^{2}+\tilde{\varepsilon}^{2}\right) e^{\frac{\omega}{3} y}+\frac{1}{|s|^{13}}
\end{aligned}
$$


Similar estimates are proved for $\left|\int \tilde{\varepsilon}_{y}^{4} \tilde{\varepsilon}^{2} e^{\omega y}\right|$ and $\left|\int \tilde{\varepsilon}_{y}^{3} \tilde{\varepsilon}^{3} e^{\omega y}\right|$. Next, for terms containing $F(\tilde{\varepsilon})-\tilde{\varepsilon}^{5}$, we argue as follows. A first observation is (using (A.1)),

$$
\left|\left(F(\tilde{\varepsilon})-\tilde{\varepsilon}^{5}\right)_{y}\right| \lesssim\left(\left|\tilde{\varepsilon}_{y}\right|+|\tilde{\varepsilon}|\right) Q, \quad\left|\left(F(\tilde{\varepsilon})-\tilde{\varepsilon}^{5}\right)_{y y}\right| \lesssim\left(\left|\tilde{\varepsilon}_{y y}\right|+\left|\tilde{\varepsilon}_{y}\right|^{2}+\left|\tilde{\varepsilon}_{y}\right|+|\tilde{\varepsilon}|\right) Q .
$$

Thus,

$$
\begin{aligned}
& \left|\int\left(F(\tilde{\varepsilon})-\tilde{\varepsilon}^{5}\right)_{y y}\left(\tilde{\varepsilon}_{y y} e^{\omega y}\right)_{y}\right| \leq C \int\left(\left|\tilde{\varepsilon}_{y y}\right|+\left|\tilde{\varepsilon}_{y}\right|^{2}+\left|\tilde{\varepsilon}_{y}\right|+|\tilde{\varepsilon}|\right)\left(\left|\tilde{\varepsilon}_{y y y}\right|+\left|\tilde{\varepsilon}_{y y}\right|\right) Q e^{\omega y} \\
& \leq \frac{1}{100} \int\left(\tilde{\varepsilon}_{y y y}^{2}+\tilde{\varepsilon}_{y y}^{2}\right) e^{\omega y}+C \int\left(\tilde{\varepsilon}_{y y}^{2}+\tilde{\varepsilon}_{y}^{4}+\tilde{\varepsilon}_{y}^{2}+\tilde{\varepsilon}^{2}\right) Q \\
& \leq \frac{1}{100} \int\left(\tilde{\varepsilon}_{y y y}^{2}+\tilde{\varepsilon}_{y y}^{2}\right) e^{\omega y}+C \int\left(\tilde{\varepsilon}_{y y}^{2}+\tilde{\varepsilon}_{y}^{2}+\tilde{\varepsilon}^{2}\right) e^{\omega y}+\frac{C}{s^{2}} .
\end{aligned}
$$

The term $\left|\int\left(F(\tilde{\varepsilon})-\tilde{\varepsilon}^{5}\right)_{y}\left(\tilde{\varepsilon}_{y y} \tilde{\varepsilon}^{4}+2 \tilde{\varepsilon}_{y}^{2} \tilde{\varepsilon}^{3}+\omega \tilde{\varepsilon}_{y} \tilde{\varepsilon}^{4}\right) e^{\omega y}\right|$ is treated similarly and easier.

Finally, terms containing $\frac{\lambda_{s}}{\lambda}$ and $\left(\frac{x_{s}}{\lambda}-1\right)$ are treated similarly as in step 1 and step 2. For example, let us consider the term $\frac{\lambda_{s}}{\lambda} \int(\Lambda Q+\Lambda \tilde{\varepsilon})_{y y} \tilde{\varepsilon}_{y y} e^{\omega y}$. We first have

$$
\left|\frac{\lambda_{s}}{\lambda} \int(\Lambda Q)_{y y} \tilde{\varepsilon}_{y y} e^{\omega y}\right|=\left|\frac{\lambda_{s}}{\lambda} \int\left((\Lambda Q)_{y y} e^{\omega y}\right)_{y y} \tilde{\varepsilon}\right| \lesssim \frac{1}{s^{2}} .
$$

Since

$$
\int(\Lambda \tilde{\varepsilon})_{y y} \tilde{\varepsilon}_{y y} e^{\omega y}=\frac{3}{2} \int \tilde{\varepsilon}_{y y}^{2} e^{\omega y}-\omega \int \tilde{\varepsilon}_{y y}^{2} y e^{\omega y}
$$

we get, for some $\frac{1}{100}<\omega^{\prime \prime}<\omega<\omega^{\prime}<\frac{1}{10}$,

$$
\left|\frac{\lambda_{s}}{\lambda} \int(\Lambda \tilde{\varepsilon})_{y y} \tilde{\varepsilon}_{y y} e^{\omega y}\right| \lesssim \frac{1}{s^{2}} \int \tilde{\varepsilon}_{y y}^{2}\left(e^{\omega^{\prime} y}+e^{\omega^{\prime \prime} y}\right) .
$$

Gathering all the previous estimates, we obtain

$$
\begin{aligned}
\frac{d}{d s} H_{2} & \lesssim-\int\left(\tilde{\varepsilon}_{y y y}^{3}+\tilde{\varepsilon}_{y y}^{2}+\tilde{\varepsilon}_{y}^{2}+\tilde{\varepsilon}^{2}\right) e^{\omega y} d y \\
& +\int\left(\tilde{\varepsilon}_{y y}^{2}+\tilde{\varepsilon}_{y}^{2}+\tilde{\varepsilon}^{2}\right)\left(e^{\frac{\omega}{3} y}+e^{\omega^{\prime \prime} y}\right)+\frac{1}{|s|^{2}} .
\end{aligned}
$$

Integrating on $\left[s_{n}, s\right]$ and passing to the limit $n \rightarrow+\infty$ using (A.8), (A.15) and (A.14), we get (A.16).

For some sequence $s_{n}^{\prime} \rightarrow-\infty$, it implies

$$
\lim _{n \rightarrow \infty} \int\left(\tilde{\varepsilon}_{y y y}^{2}+\tilde{\varepsilon}_{y y}^{2}+\varepsilon_{y}^{2}+\tilde{\varepsilon}^{2}\right)\left(s_{n}^{\prime}, y\right) e^{\omega y} d y=0 .
$$

Note also that by standard arguments, (A.16) implies directly that

$$
\left\|\varepsilon_{y}^{2} e^{\omega y}\right\|_{L^{\infty}} \lesssim \frac{1}{|s|}
$$

step 4 We claim that for all $\frac{9}{B}<\omega<\frac{1}{10}$, for $|s|$ large,

$$
\int \tilde{\varepsilon}_{y y y}^{2}(s, y) e^{\omega y} d y+\int_{-\infty}^{s} \int\left(\partial_{y}^{4} \tilde{\varepsilon}\right)^{2}\left(s^{\prime}, y\right) e^{\omega y} d y d s^{\prime} \lesssim \frac{1}{|s|} .
$$

Define

$$
H_{3}(s)=\frac{1}{2} \int \tilde{\varepsilon}_{y y y}^{2} e^{\omega y}
$$


Then,

$$
\begin{aligned}
& \frac{d}{d s} H_{3}=\int \tilde{\varepsilon}_{y y y s} \tilde{\varepsilon}_{y y y} e^{\omega y}=\int\left(-\tilde{\varepsilon}_{y y}+\tilde{\varepsilon}-F(\tilde{\varepsilon})\right)_{y y y y} \varepsilon_{y y y} e^{\omega y} \\
& +\frac{\lambda_{s}}{\lambda} \int(\Lambda Q+\Lambda \tilde{\varepsilon})_{y y y} \tilde{\varepsilon}_{y y y} e^{\omega y}+\left(\frac{x_{s}}{\lambda}-1\right) \int\left(Q^{\prime}+\tilde{\varepsilon}_{y}\right)_{y y y} \tilde{\varepsilon}_{y y y} e^{\omega y} \\
& =-\frac{3}{2} \omega \int \tilde{\varepsilon}_{y y y y}^{2} e^{\omega y}-\frac{\omega}{2}\left(1-\omega^{2}\right) \int \tilde{\varepsilon}_{y y y}^{2} e^{\omega y}+\int(F(\tilde{\varepsilon}))_{y y y}\left(\varepsilon_{y y y} e^{\omega y}\right)_{y} \\
& +\frac{\lambda_{s}}{\lambda} \int(\Lambda Q+\Lambda \tilde{\varepsilon})_{y y y} \tilde{\varepsilon}_{y y y} e^{\omega y}+\left(\frac{x_{s}}{\lambda}-1\right) \int\left(Q^{\prime}+\tilde{\varepsilon}_{y}\right)_{y y y} \tilde{\varepsilon}_{y y y} e^{\omega y}
\end{aligned}
$$

The last two terms $\frac{\lambda_{s}}{\lambda} \int(\Lambda Q+\Lambda \tilde{\varepsilon})_{y y y} \tilde{\varepsilon}_{y y y} e^{\omega y}$ and $\left(\frac{x_{s}}{\lambda}-1\right) \int\left(Q^{\prime}+\tilde{\varepsilon}_{y}\right)_{y y y} \tilde{\varepsilon}_{y y y} e^{\omega y}$ are treated exactly as in the previous steps and thus we omit the estimates.

We focus on the nonlinear term $\int(F(\tilde{\varepsilon}))_{y y y}\left(\varepsilon_{y y y} e^{\omega y}\right)_{y}$. Expanding $F(\tilde{\varepsilon})=5 Q^{4} \tilde{\varepsilon}+$ $10 Q^{3} \tilde{\varepsilon}^{2}+10 Q^{2} \tilde{\varepsilon}^{3}+5 Q \tilde{\varepsilon}^{4}+\tilde{\varepsilon}^{5}$ and integrating by parts, we obtain many different terms. We check the worst terms and we claim that the other terms can be checked similarly. See also Section 3.4 in 25] for similar arguments.

First, we remark that the following term which is only quadratic in $\tilde{\varepsilon}$, is easily controlled

$$
\left|\int \tilde{\varepsilon}_{y y y}^{2}\left(Q^{4}\right)^{\prime} e^{\omega y}\right| \lesssim \int \tilde{\varepsilon}_{y y y}^{2} e^{\omega y}
$$

Second, we treat some terms coming from $\tilde{\varepsilon}^{5}$ :

$$
\begin{aligned}
\left|\int \tilde{\varepsilon}_{y y y}^{2} \tilde{\varepsilon}_{y} \tilde{\varepsilon}^{3} e^{\omega y}\right| & \lesssim\|\tilde{\varepsilon}\|_{L^{\infty}}^{3}\left\|\tilde{\varepsilon}_{y} e^{\frac{\omega}{2} y}\right\|_{L^{\infty}} \int \tilde{\varepsilon}_{y y y}^{2} e^{\frac{\omega}{2} y} \lesssim \frac{1}{|s|^{2}} \int \tilde{\varepsilon}_{y y y}^{2} e^{\frac{\omega}{2} y} \\
\left|\int \tilde{\varepsilon}_{y y}^{3} \tilde{\varepsilon}_{y} \tilde{\varepsilon}^{2} e^{\omega y}\right| & \lesssim\|\tilde{\varepsilon}\|_{L^{\infty}}^{2}\left\|\tilde{\varepsilon}_{y} e^{\frac{\omega}{4} y}\right\|_{L^{\infty}}\left|\int \tilde{\varepsilon}_{y y}^{3} e^{\frac{3 \omega}{4} y}\right| \\
& \lesssim \frac{1}{|s|^{\frac{3}{2}}}\left(\int\left(\tilde{\varepsilon}_{y y y}^{2}+\tilde{\varepsilon}_{y y}^{2}\right) e^{\frac{\omega}{2} y}\right)^{\frac{1}{4}}\left(\int \tilde{\varepsilon}_{y y}^{2} e^{\frac{\omega}{2} y}\right)^{\frac{5}{4}} \\
& \lesssim \frac{1}{|s|^{\frac{11}{3}}}+\int \tilde{\varepsilon}_{y y y}^{2} e^{\frac{\omega}{2} y} \cdot m \\
\left|\int \tilde{\varepsilon}_{y y}^{2} \tilde{\varepsilon}_{y}^{3} \tilde{\varepsilon} e^{\omega y}\right| & \lesssim\left\|\tilde{\varepsilon}_{L^{\infty}}\right\| \tilde{\varepsilon}_{y} e^{\frac{\omega}{5} y} \|_{L^{\infty}}^{3}\left|\int \tilde{\varepsilon}_{y y}^{2} e^{\frac{2 \omega}{5} y}\right| \lesssim \frac{1}{|s|^{3}} .
\end{aligned}
$$

Thus, we get

$$
\frac{d}{d s} H_{3} \lesssim-\int\left(\partial_{y}^{4} \varepsilon\right)^{2} e^{\omega y}+\frac{1}{|s|^{2}}+\int\left(\tilde{\varepsilon}_{y y y}^{2}+\tilde{\varepsilon}_{y y}^{2}+\tilde{\varepsilon}_{y}^{2}+\tilde{\varepsilon}^{2}\right)\left(e^{\frac{2 \omega}{5} y}+e^{\omega y}\right) .
$$

Integrating on $\left[s_{n}^{\prime}, s\right]$ and passing to the limit as $n \rightarrow+\infty$, using (A.17) and (A.16), we obtain (A.19), for the following range of values of $\omega$ : $\frac{15}{2 B}<\omega<\frac{1}{10}$.

\section{Appendix B. Proof of Lemma 6.3}

For simplicity of notation, we denote $\varepsilon_{v_{n}}, \lambda_{v_{n}}, b_{v_{n}}$ and $x_{v_{n}}$ simply by $\varepsilon, \lambda, b$ and $x$. 
step 1 Algebraic computations. We follow closely the computations of the proof of Proposition 3.1 in [33]. First,

$$
\begin{aligned}
\frac{d}{d s} \mathcal{F}_{B} & =\frac{1}{\lambda^{2}}\left(\frac{1}{2} \int\left[\varepsilon_{y}^{2} \tilde{\phi}_{B}^{\prime}+\frac{\lambda^{2}}{\lambda_{0}^{2}} \varepsilon^{2} \phi_{B}^{\prime}-\frac{1}{3}\left(\left(\varepsilon+Q_{b}\right)^{6}-Q_{b}^{6}-6 \varepsilon Q_{b}^{5}\right) \tilde{\phi}_{B}^{\prime}\right]\right. \\
& +2 \int \tilde{\phi}_{B}\left(\varepsilon_{y}\right)_{s} \varepsilon_{y}+2 \varepsilon_{s}\left[\frac{\lambda^{2}}{\lambda_{0}^{2}} \varepsilon \phi_{B}-\tilde{\phi}_{B}\left(\left(\varepsilon+Q_{b}\right)^{5}-Q_{b}^{5}\right)\right] \\
& \left.-2 \int \tilde{\phi}_{B}\left(Q_{b}\right)_{s}\left(\left(\varepsilon+Q_{b}\right)^{5}-Q_{b}^{5}-5 \varepsilon Q_{b}^{4}\right)\right) \\
& -2 \frac{\lambda_{s}}{\lambda^{3}} \int\left[\varepsilon_{y}^{2} \tilde{\phi}_{B}-\frac{1}{3}\left(\left(\varepsilon+Q_{b}\right)^{6}-Q_{b}^{6}-6 \varepsilon Q_{b}^{5}\right) \tilde{\phi}_{B}\right]-2 \frac{\left(\lambda_{0}\right)_{s}}{\lambda_{0}^{3}} \int \varepsilon^{2} \phi_{B} \\
& =\frac{1}{\lambda^{2}}\left(f_{1}+f_{2}+f_{3}+f_{4}\right),
\end{aligned}
$$

where

$$
\begin{aligned}
f_{1} & =\frac{1}{2} \int\left[\varepsilon_{y}^{2} \tilde{\phi}_{B}^{\prime}+\varepsilon^{2} \phi_{B}^{\prime}-\frac{1}{3}\left(\left(\varepsilon+Q_{b}\right)^{6}-Q_{b}^{6}-6 \varepsilon Q_{b}^{5}\right) \tilde{\phi}_{B}^{\prime}\right] \\
& +2 \int\left(\varepsilon_{s}-\frac{\lambda_{s}}{\lambda} \Lambda \varepsilon\right)\left(-\left(\tilde{\phi}_{B} \varepsilon_{y}\right)_{y}+\varepsilon \phi_{B}-\tilde{\phi}_{B}\left(\left(\varepsilon+Q_{b}\right)^{5}-Q_{b}^{5}\right)\right), \\
f_{2} & =2\left(1-\frac{\lambda^{2}}{\lambda_{0}^{2}}\right) \int \varepsilon_{s} \varepsilon \phi_{B}-2 \frac{\left(\lambda_{0}\right)_{s}}{\lambda_{0}} \int \varepsilon^{2} \phi_{B} \\
f_{3} & =2 \frac{\lambda_{s}}{\lambda} \int \Lambda \varepsilon\left(-\left(\tilde{\phi}_{B} \varepsilon_{y}\right)_{y}+\varepsilon \phi_{B}-\tilde{\phi}_{B}\left(\left(\varepsilon+Q_{b}\right)^{5}-Q_{b}^{5}\right)\right) \\
& -2 \frac{\lambda_{s}}{\lambda} \int\left[\varepsilon_{y}^{2} \tilde{\phi}_{B}-\frac{1}{3}\left(\left(\varepsilon+Q_{b}\right)^{6}-Q_{b}^{6}-6 \varepsilon Q_{b}^{5}\right) \tilde{\phi}_{B}\right] \\
f_{4} & =-2 \int \tilde{\phi}_{B}\left(Q_{b}\right)_{s}\left(\left(\varepsilon+Q_{b}\right)^{5}-Q_{b}^{5}-5 \varepsilon Q_{b}^{4}\right) .
\end{aligned}
$$

We use the equation of $\varepsilon$ under the following form

$$
\begin{aligned}
\varepsilon_{s}-\frac{\lambda_{s}}{\lambda} \Lambda \varepsilon & =\left(-\varepsilon_{y y}+\varepsilon-\left(\varepsilon+Q_{b}\right)^{5}+Q_{b}^{5}\right)_{y} \\
& +\left(\frac{\lambda_{s}}{\lambda}+b\right) \Lambda Q_{b}+\left(\frac{x_{s}}{\lambda}-1\right)\left(Q_{b}+\varepsilon\right)_{y}+\Phi_{b}+\Psi_{b}
\end{aligned}
$$

where $\Phi_{b}=-b_{s}\left(\chi_{b}+\gamma y\left(\chi_{b}\right)_{y}\right) P$ and $-\Psi_{b}=\left(Q_{b}^{\prime \prime}-Q_{b}+Q_{b}^{5}\right)^{\prime}+b \Lambda Q_{b}$. 
step 2 Control of $f_{1}$.

$$
\begin{aligned}
\mathrm{f}_{1} & =\frac{1}{2} \int\left[\varepsilon_{y}^{2} \tilde{\phi}_{B}^{\prime}+\varepsilon^{2} \phi_{B}^{\prime}-\frac{1}{3}\left(\left(\varepsilon+Q_{b}\right)^{6}-Q_{b}^{6}-6 \varepsilon Q_{b}^{5}\right) \tilde{\phi}_{B}^{\prime}\right] \\
& +2 \int\left(-\varepsilon_{y y}+\varepsilon-\left(\left(\varepsilon+Q_{b}\right)^{5}-Q_{b}^{5}\right)\right)_{y}\left(-\left(\tilde{\phi}_{B} \varepsilon_{y}\right)_{y}+\varepsilon \phi_{B}-\tilde{\phi}_{B}\left[\left(Q_{b}+\varepsilon\right)^{5}-Q_{b}^{5}\right]\right) \\
& +2\left(\frac{\lambda_{s}}{\lambda}+b\right) \int \Lambda Q_{b}\left(-\left(\tilde{\phi}_{B} \varepsilon_{y}\right)_{y}+\varepsilon \phi_{B}-\tilde{\phi}_{B}\left(\left(\varepsilon+Q_{b}\right)^{5}-Q_{b}^{5}\right)\right) \\
& +2\left(\frac{x_{s}}{\lambda}-1\right) \int\left(Q_{b}+\varepsilon\right)_{y}\left(-\left(\tilde{\phi}_{B} \varepsilon_{y}\right)_{y}+\varepsilon \phi_{B}-\tilde{\phi}_{B}\left(\left(\varepsilon+Q_{b}\right)^{5}-Q_{b}^{5}\right)\right) \\
& +2 \int \Phi_{b}\left(-\left(\tilde{\phi}_{B} \varepsilon_{y}\right)_{y}+\varepsilon \phi_{B}-\tilde{\phi}_{B}\left(\left(\varepsilon+Q_{b}\right)^{5}-Q_{b}^{5}\right)\right) \\
& +2 \int \Psi_{b}\left(-\left(\tilde{\phi}_{B} \varepsilon_{y}\right)_{y}+\varepsilon \phi_{B}-\tilde{\phi}_{B}\left(\left(\varepsilon+Q_{b}\right)^{5}-Q_{b}^{5}\right)\right) \\
& =\mathrm{f}_{1,1}+\mathrm{f}_{1,2}+\mathrm{f}_{1,3}+\mathrm{f}_{1,4}+\mathrm{f}_{1,5} .
\end{aligned}
$$

As in [33, we obtain after some computations,

$$
\begin{aligned}
f_{1,1} & =-\int\left[3 \tilde{\phi}_{B}^{\prime} \varepsilon_{y y}^{2}+\left(3 \phi_{B}^{\prime}+\frac{1}{2} \tilde{\phi}_{B}^{\prime}-\tilde{\phi}_{B}^{\prime \prime \prime}\right) \varepsilon_{y}^{2}+\left(\frac{1}{2} \phi_{B}^{\prime}-\phi_{B}^{\prime \prime \prime}\right) \varepsilon^{2}\right] \\
& -\frac{1}{6} \int\left(\left(\varepsilon+Q_{b}\right)^{6}-Q_{b}^{6}-6 \varepsilon Q_{b}^{5}\right) \tilde{\phi}_{B}^{\prime} \\
& -2 \int\left[\frac{\left(\varepsilon+Q_{b}\right)^{6}}{6}-\frac{Q_{b}^{6}}{6}-Q_{b}^{5} \varepsilon-\left(\left(\varepsilon+Q_{b}\right)^{5}-Q_{b}^{5}\right) \varepsilon\right]\left(\phi_{B}^{\prime}-\tilde{\phi}_{B}^{\prime}\right) \\
& +2 \int\left[\left(\varepsilon+Q_{b}\right)^{5}-Q_{b}^{5}-5 Q_{b}^{4} \varepsilon\right]\left(Q_{b}\right)_{y}\left(\tilde{\phi}_{B}-\phi_{B}\right) \\
& +10 \int \tilde{\phi}_{B}^{\prime} \varepsilon_{y}\left\{\left(Q_{b}\right)_{y}\left[\left(Q_{b}+\varepsilon\right)^{4}-Q_{b}^{4}\right]+\left(Q_{b}+\varepsilon\right)^{4} \varepsilon_{y}\right\} \\
& +\int \tilde{\phi}_{B}^{\prime}\left[-2 \varepsilon_{y y}+2 \varepsilon-\left(\left(\varepsilon+Q_{b}\right)^{5}-Q_{b}^{5}\right)\right]\left[\left(\varepsilon+Q_{b}\right)^{5}-Q_{b}^{5}\right]
\end{aligned}
$$

Using the following estimates (see [33] for more details),

$$
\begin{aligned}
& \tilde{\phi}_{B}^{\prime \prime \prime} \lesssim \frac{1}{B^{2}} \phi_{B}^{\prime}, \quad \phi_{B}^{\prime \prime \prime} \lesssim \frac{1}{B^{2}} \phi_{B}^{\prime}, \quad \text { for all } y \in \mathbb{R}, \\
& \left|Q_{b}(y)\right|+\left|\left(Q_{b}\right)_{y}(y)\right| \lesssim e^{-|y|}+|b|, \quad \text { for all } y \in \mathbb{R}, \\
& \int \varepsilon^{6} \phi_{B}^{\prime} \lesssim \delta\left(\alpha^{*}\right) \int\left(\varepsilon_{y}^{2}+\varepsilon^{2}\right) \phi_{B}^{\prime}, \\
& \int \varepsilon_{y}^{2} \varepsilon^{4} \tilde{\phi}_{B}^{\prime} \leq \delta\left(\alpha^{*}\right)\left(\int \varepsilon_{y y}^{2} \tilde{\phi}_{B}^{\prime}+\int\left(\varepsilon_{y}^{2}+\varepsilon^{2}\right) \phi_{B}^{\prime}\right),
\end{aligned}
$$

and the bound on the $L^{2}$ norm of $\varepsilon$ (see Lemma 6.2), we obtain for $B$ large and $\alpha^{*}$ small:

$$
\begin{aligned}
f_{1,1} & \leq-\int \tilde{\phi}_{B}^{\prime} \varepsilon_{y y}^{2}-\frac{1}{4} \int\left(\varepsilon_{y}^{2}+\varepsilon^{2}\right) \phi_{B}^{\prime}+C \int\left(\varepsilon_{y}^{2}+\varepsilon^{2}\right) e^{-\frac{|y|}{10}}+C|b|^{4} \\
& \leq-\int \tilde{\phi}_{B}^{\prime} \varepsilon_{y y}^{2}-\frac{1}{4} \int\left(\varepsilon_{y}^{2}+\varepsilon^{2}\right) \phi_{B}^{\prime}+C \int\left(\varepsilon_{y}^{2}+\varepsilon^{2}\right) \frac{\varphi_{B}}{1+y_{+}^{2}}+C|b|^{4} .
\end{aligned}
$$


Next,

$$
\begin{aligned}
f_{1,2} & =2\left(\frac{\lambda_{s}}{\lambda}+b\right) \int \Lambda Q(L \varepsilon)-2\left(\frac{\lambda_{s}}{\lambda}+b\right) \int \varepsilon\left(1-\phi_{B}\right) \Lambda Q \\
& \left.+2 b\left(\frac{\lambda_{s}}{\lambda}+b\right) \int \Lambda\left(\chi_{b} P\right)\left(-\left(\tilde{\phi}_{B} \varepsilon_{y}\right)_{y}+\varepsilon \phi_{B}-\tilde{\phi}_{B}\left[\left(Q_{b}+\varepsilon\right)^{5}-Q_{b}^{5}\right)\right]\right) \\
& +2\left(\frac{\lambda_{s}}{\lambda}+b\right) \int \Lambda Q\left(-\left(\tilde{\phi}_{B}\right)_{y} \varepsilon_{y}-\left(1-\tilde{\phi}_{B}\right) \varepsilon_{y y}+\left(1-\tilde{\phi}_{B}\right)\left[\left(Q_{b}+\varepsilon\right)^{5}-Q_{b}^{5}\right]\right) \\
& +2\left(\frac{\lambda_{s}}{\lambda}+b\right) \int \Lambda Q\left[\left(Q_{b}+\varepsilon\right)^{5}-Q_{b}^{5}-5 Q^{4} \varepsilon\right] .
\end{aligned}
$$

The main term $\int \Lambda Q(L \varepsilon)$ is zero by the orthogonality conditions on $\varepsilon$ and the other terms are controled as in [33] using (2.29), (B.2), (B.3) and (B.5), to obtain

$$
\left|f_{1,2}\right| \leq \frac{1}{100} \int\left(\varepsilon_{y}^{2}+\varepsilon^{2}\right) \phi_{B}^{\prime}+C \int\left(\varepsilon_{y}^{2}+\varepsilon^{2}\right) \frac{\varphi_{B}}{1+y_{+}^{2}}+C|b|^{4} .
$$

The next term is

$$
\begin{aligned}
f_{1,3} & =2\left(\frac{x_{s}}{\lambda}-1\right) \int \frac{1}{6} \tilde{\phi}_{B}^{\prime}\left[\left(Q_{b}+\varepsilon\right)^{6}-Q_{b}^{6}-6 Q_{b}^{5} \varepsilon\right] \\
& +2\left(\frac{x_{s}}{\lambda}-1\right) \int\left(b \chi_{b} P+\varepsilon\right)_{y}\left[-\tilde{\phi}_{B}^{\prime} \varepsilon_{y}-\tilde{\phi}_{B} \varepsilon_{y y}+\varepsilon \phi_{B}\right] \\
& +2\left(\frac{x_{s}}{\lambda}-1\right) \int Q^{\prime}\left[L \varepsilon-\tilde{\phi}_{B}^{\prime} \varepsilon_{y}+\left(1-\tilde{\phi}_{B}\right) \varepsilon_{y y}-\varepsilon\left(1-\phi_{B}\right)\right] \\
& +10\left(\frac{x_{s}}{\lambda}-1\right) \int \varepsilon \tilde{\phi}_{B}\left(Q_{b}^{4}\left(Q_{b}\right)_{y}-Q^{4} Q_{y}\right)
\end{aligned}
$$

Using $L Q^{\prime}=0$ and arguing similarly as before, we obtain

$$
\left|f_{1,3}\right| \leq \frac{1}{100} \int\left(\varepsilon_{y}^{2}+\varepsilon^{2}\right) \phi_{B}^{\prime}+C \int\left(\varepsilon_{y}^{2}+\varepsilon^{2}\right) \frac{\varphi_{B}}{1+y_{+}^{2}}+C|b|^{4} .
$$

step 3 Control of $f_{2}$.

First, by (6.14), we have

$$
-\frac{\left(\lambda_{0}\right)_{s}}{\lambda_{0}} \int \varepsilon^{2} \phi_{B}<0
$$

Next, by the definition of $\lambda_{0}$ in Lemma 2.10, we have

$$
\left|1-\frac{\lambda^{2}}{\lambda_{0}^{2}}\right| \lesssim \mathcal{N}^{\frac{1}{2}} \lesssim \delta\left(\alpha^{*}\right)
$$

and thus, proceeding for $\int \varepsilon_{s} \varepsilon \phi_{B}$ as in the previous step, we find

$$
\left|1-\frac{\lambda^{2}}{\lambda_{0}^{2}}\right|\left|\int \varepsilon_{s} \varepsilon \phi_{B}\right| \leq \frac{1}{100} \int\left(\varepsilon_{y}^{2}+\varepsilon^{2}\right) \phi_{B}^{\prime}+C \int\left(\varepsilon_{y}^{2}+\varepsilon^{2}\right) \frac{\varphi_{B}}{1+y_{+}^{2}}+C|b|^{4} .
$$


step 4 Control of $f_{3}$. From computations in [33,

$$
\begin{aligned}
f_{3} & =\frac{\lambda_{s}}{\lambda} \int\left[2 \tilde{\phi}_{B}-y \tilde{\phi}_{B}^{\prime}\right] \varepsilon_{y}^{2}-\frac{\lambda_{s}}{\lambda} \int y \tilde{\phi}_{B}^{\prime} \varepsilon^{2} \\
& -\frac{1}{3} \frac{\lambda_{s}}{\lambda} \int\left[2 \tilde{\phi}_{B}-y \tilde{\phi}_{B}^{\prime}\right]\left[\left(\varepsilon+Q_{b}\right)^{6}-Q_{b}^{6}-6 Q_{b}^{5} \varepsilon\right] \\
& +2 \frac{\lambda_{s}}{\lambda} \int \tilde{\phi}_{B} \Lambda Q_{b}\left(\left(\varepsilon+Q_{b}\right)^{5}-Q_{b}^{5}-5 Q_{b}^{4} \varepsilon\right) \\
& -2 \frac{\lambda_{s}}{\lambda} \int\left[\varepsilon_{y}^{2} \tilde{\phi}_{B}-\frac{1}{3}\left(\left(\varepsilon+Q_{b}\right)^{6}-Q_{b}^{6}-6 \varepsilon Q_{b}^{5}\right) \tilde{\phi}_{B}\right] .
\end{aligned}
$$

After simplification of the last line with terms in the first and second lines, we obtain

$$
\begin{aligned}
f_{3} & =-\frac{\lambda_{s}}{\lambda} \int y \tilde{\phi}_{B}^{\prime}\left[\varepsilon_{y}^{2}+\varepsilon^{2}-\frac{1}{3}\left(\left(\varepsilon+Q_{b}\right)^{6}-Q_{b}^{6}-6 Q_{b}^{5} \varepsilon\right)\right] \\
& +2 \frac{\lambda_{s}}{\lambda} \int \tilde{\phi}_{B} \Lambda Q_{b}\left(\left(\varepsilon+Q_{b}\right)^{5}-Q_{b}^{5}-5 Q_{b}^{4} \varepsilon\right) .
\end{aligned}
$$

For this term we observe, from the definition of $\phi_{B}$ and $\tilde{\phi}_{B}$,

$$
\int|y| \tilde{\phi}_{B}^{\prime}\left(\varepsilon_{y}^{2}+\varepsilon^{2}+|\varepsilon|^{6}\right) \lesssim \int\left(\varepsilon_{y}^{2}+\varepsilon^{2}\right) \phi_{B}^{\prime}
$$

and $\left|\frac{\lambda_{s}}{\lambda}\right| \lesssim \delta\left(\alpha^{*}\right)$. The other terms in the expression of $f_{3}$ are treated as before, so that we obtain:

$$
\left|f_{3}\right| \leq \frac{1}{100} \int\left(\varepsilon_{y}^{2}+\varepsilon^{2}\right) \phi_{B}^{\prime}+C \int\left(\varepsilon_{y}^{2}+\varepsilon^{2}\right) \frac{\varphi_{B}}{1+y_{+}^{2}}+C|b|^{4}
$$

step 5 Control of $f_{4}$. Arguing exactly as in [33] (using (2.30), we obtain

$$
\left|f_{4}\right| \leq \frac{1}{100} \int\left(\varepsilon_{y}^{2}+\varepsilon^{2}\right) \phi_{B}^{\prime}+C \int\left(\varepsilon_{y}^{2}+\varepsilon^{2}\right) \frac{\varphi_{B}}{1+y_{+}^{2}}+C|b|^{4} .
$$

Gathering these estimates, we get (6.25).

step 6 Proof of (6.26). This is a standard fact by localization arguments (see e.g. Appendix A of [28]).

\section{References}

[1] V. Banica, R. Carles and T. Duyckaerts, Minimal blow-up solutions to the mass-critical inhomogeneous focusing NLS equation, Comm. Partial Differential Equations 36 (2011), no. 3, $487-531$.

[2] H. Berestycki and T. Cazenave, Instabilité des états stationnaires dans les équations de Schrödinger et de Klein-Gordon non linéaires. (French. English summary) [Instability of stationary states in nonlinear Schrödinger and Klein-Gordon equations] C. R. Acad. Sci. Paris Sér. I Math. 293 (1981), no. 9, 489-492.

[3] J. Bourgain and W. Wang, Construction of blowup solutions for the nonlinear Schrödinger equation with critical nonlinearity, Ann. Scuola Norm. Sup. Pisa Cl. Sci. (4) 25 (1997), 197215 (1998).

[4] N. Burq, P. Gérard and N. Tzvetkov, Two singular dynamics of the nonlinear Schrödinger equation on a plane domain, Geom. Funct. Anal. 13 (2003), 1-19.

[5] R. Côte, Construction of solutions to the $L^{2}$-critical $\mathrm{KdV}$ equation with a given asymptotic behaviour, Duke Math. J. 138 (2007), 487-531.

[6] R. Côte, Y. Martel and F. Merle, Construction of multi-soliton solutions for the $L^{2}$ supercritical gKdV and NLS equations, Rev. Mat. Iberoamericana, 27 (2011), 273-302.

[7] R. Côte and H. Zaag, Construction of a multi-soliton blow-up solution to the semilinear wave equation in one space dimension, Comm. Pure Appl. Math. 66 (2013) no. 10, 1541-1581. 
[8] T. Duyckaerts, C. Kenig and F. Merle, Universality of blow-up profile for small radial type II blow-up solutions of energy-critical wave equation, J. Eur. Math. Soc. 13 (2011), no. 3, 533-599.

[9] T. Duyckaerts, C. Kenig and F. Merle, Universality of the blow-up profile for small type II blowup solutions of energy-critical wave equation: the non-radial case, J. Eur. Math. Soc. 14 (2012), no. 5, 1389-1454.

[10] T. Duyckaerts and F. Merle, Dynamics of threshold solutions for energy-critical wave equation. Int. Math. Res. Pap. IMRP 2007, Art. ID rpn002, 67 pp. (2008).

[11] T. Duyckaerts and F. Merle, Dynamic of threshold solutions for energy-critical NLS, Geom. Funct. Anal. 18 (2009), 1787-1840.

[12] T. Duyckaerts and S. Roudenko, Threshold solutions for the focusing 3D cubic Schrödinger equation, Rev. Mat. Iberoam. 26 (2010), 1-56.

[13] T. Kato, On the Cauchy problem for the (generalized) Korteweg-de Vries equation. Studies in applied mathematics, 93-128, Adv. Math. Suppl. Stud., 8, Academic Press, New York, 1983.

[14] C.E. Kenig and F. Merle, Global well-posedness, scattering and blow-up for the energy-critical, focusing, nonlinear Schrödinger equation in the radial case. Invent. Math. 166 (2006) 645-675.

[15] C.E. Kenig, G. Ponce and L. Vega, Well-posedness and scattering results for the generalized Korteweg-de Vries equation via the contraction principle, Comm. Pure Appl. Math. 46, (1993) $527-620$.

[16] C.E. Kenig, G. Ponce and L. Vega, On the concentration of blow up solutions for the generalized KdV equation critical in $L^{2}$. Nonlinear wave equations (Providence, RI, 1998), 131-156, Contemp. Math., 263, Amer. Math. Soc., Providence, RI, 2000.

[17] C.E. Kenig and F. Merle, Global well-posedness, scattering and blow-up for the energy-critical, focusing, non-linear Schrödinger equation in the radial case, Invent. Math. 166 (2006), 645675.

[18] R. Killip, S. Kwon, S. Shao and M. Visan, On the mass-critical generalized KdV equation, Discrete Contin. Dyn. Syst. 32 (2012), 191-221.

[19] J. Krieger, Y. Martel and P. Raphaël, Two-soliton solutions to the three-dimensional gravitational Hartree equation, Comm. Pure Appl. Math. 62 (2009), 1501-1550.

[20] J. Krieger, E. Lenzmann and P. Raphaël, Nondispersive solutions to the $L^{2}$-critical half-wave equation. Arch. Ration. Mech. Anal. 209 (2013), no. 1, 61-129.

[21] J. Krieger, K. Nakanishi and W. Schlag, Global dynamics away from the ground state for the energy-critical nonlinear wave equation, Amer. J. Math. 135 (2013), no. 4, 935, Äì65.

[22] J. Krieger and W. Schlag, Non-generic blow-up solutions for the critical focusing NLS in 1-D, J. Eur. Math. Soc. 11 (2009), 1-125.

[23] J. Krieger, W. Schlag and D. Tataru, Renormalization and blow up for charge one equivariant critical wave maps, Invent. Math. 171 (2008), 543-615.

[24] J. Krieger, W. Schlag and D. Tataru, Slow blow-up solutions for the $H^{1}\left(\mathbb{R}^{3}\right)$ critical focusing semilinear wave equation, Duke Math. J. 147 (2009), 1-53.

[25] Y. Martel, Asymptotic $N$-soliton-like solutions of the subcritical and critical generalized Korteweg-de Vries equations, Amer. J. Math. 127 (2005), 1103-1140.

[26] Y. Martel and F. Merle, A Liouville theorem for the critical generalized Korteweg-de Vries equation, J. Math. Pures Appl. 79 (2000), 339-425.

[27] Y. Martel and F. Merle, Instability of solitons for the critical generalized Korteweg-de Vries equation. Geom. Funct. Anal. 11 (2001), 74-123.

[28] Y. Martel and F. Merle, Stability of blow up profile and lower bounds for blow up rate for the critical generalized KdV equation, Ann. of Math. 155 (2002), 235-280.

[29] Y. Martel and F. Merle, Blow up in finite time and dynamics of blow up solutions for the $L^{2}$-critical generalized KdV equation, J. Amer. Math. Soc. 15 (2002), 617-664.

[30] Y. Martel and F. Merle, Nonexistence of blow-up solution with minimal $L^{2}$-mass for the critical gKdV equation, Duke Math. J. 115 (2002), 385-408.

[31] Y. Martel and F. Merle, Multi solitary waves for nonlinear Schrödinger equations. Ann. Inst. H. Poincaré Anal. Non Linéaire 23 (2006), 849-864.

[32] Y. Martel and F. Merle, Description of the collision of two solitons for the quartic gKdV equation, Annals of Math. 174 (2011), 757-857.

[33] Y. Martel, F. Merle and P. Raphaël, Blow up for the critical generalized Korteweg-de Vries equation. I: Dynamics near the soliton, Acta Math. 212 (2014), no. 1, 59-140.

[34] Y. Martel, F. Merle and P. Raphaël, Blow up for the critical gKdV equation. III: Exotic regimes. To appear in Annali della Scuola Normale Superiore di Pisa 
[35] Y. Martel, F. Merle and T.-P. Tsai, Stability in $H^{1}$ of the sum of $K$ solitary waves for some nonlinear Schrödinger equations, Duke Math. J. 133 (2006), 405-466.

[36] F. Merle, Construction of solutions with exactly $k$ blow-up points for the Schrödinger equation with critical nonlinearity, Comm. Math. Phys. 129 (1990), 223-240.

[37] F. Merle, Determination of blow-up solutions with minimal mass for nonlinear Schrödinger equations with critical power, Duke Math. J. 69 (1993), 427-454.

[38] F. Merle, Nonexistence of minimal blow-up solutions of equations $i u_{t}=-\Delta u-k(x)|u|^{\frac{4}{N}} u$ in $\mathbb{R}^{N}$, Ann. Inst. H. Poincaré Phys. Théor. 64 (1996), 33-85.

[39] F. Merle, Existence of blow-up solutions in the energy space for the critical generalized KdV equation. J. Amer. Math. Soc. 14 (2001), 555-578.

[40] F. Merle and P. Raphaël, Sharp upper bound on the blow up rate for the critical nonlinear Schrödinger equation, Geom. Func. Anal. 13 (2003), 591-642.

[41] F. Merle and P. Raphaël, On universality of blow-up profile for $L^{2}$ critical nonlinear Schrödinger equation. Invent. Math. 156 (2004), 565-672.

[42] F. Merle and P. Raphaël, The blow up dynamics and upper bound on the blow up rate for the critical nonlinear Schrödinger equation, Ann. of Math. 161 (2005), 157-222.

[43] F. Merle and P. Raphaël, Profiles and quantization of the blow up mass for critical nonlinear Schrödinger equation, Commun. Math. Phys. 253 (2005), 675-704.

[44] F. Merle and P. Raphaël, On a sharp lower bound on the blow-up rate for the $L^{2}$ critical nonlinear Schrödinger equation, J. Amer. Math. Soc. 19 (2006), 37-90.

[45] F. Merle, P. Raphaël and J. Szeftel, The instability of Bourgain-Wang solutions for the $L^{2}$ critical NLS, Amer. J. Math. 135 (2013), no. 4, 967-1017.

[46] F. Merle, P. Raphaël and I. Rodnianski, Blow up dynamics for smooth data equivariant solutions to the energy critical Schrodinger map problem, Invent. Math. 193 (2013), no. 2, $249-365$.

[47] K. Nakanishi and W. Schlag, Global dynamics above the ground state energy for the focusing nonlinear Klein-Gordon equation, J. Differential Equations 250 (2011), 2299-2333.

[48] K. Nakanishi and W. Schlag, Global dynamics above the ground state energy for the cubic NLS equation in 3D, Calc. Var. Partial Differential Equations 44 (2012), no. 1-2, 1-45.

[49] P. Raphaël, Stability of the log-log bound for blow up solutions to the critical non linear Schrödinger equation, Math. Ann. 331 (2005), 577-609.

[50] P. Raphaël, Stability and blow up for the nonlinear Schrodinger equation, Lecture notes for the Clay summer school on evolution equations, ETH, Zurich (2008), http://www.math.univtoulouse.fr/ raphael/Teaching.html

[51] P. Raphaël and I. Rodnianski, Stable blow up dynamics for the critical co-rotational Wave Maps and equivariant Yang-Mills problems, Publ. Math. Inst. Hautes Etudes Sci. 115 (2012), $1-122$.

[52] P. Raphaël and R. Schweyer, Stable blow up dynamics for the 1-corotational harmonic heat flow, Comm. Pure Appl. Math. 66 (2013), no. 3, 414-480.

[53] P. Raphaël and J. Szeftel, Existence and uniqueness of minimal blow up solutions to an inhomogeneous mass critical NLS, J. Amer. Math. Soc. 24 (2011), no. 2, 471-546.

[54] S. Shao, The linear profile decomposition for the Airy equation and the existence of maximizers for the Airy Strichartz inequality, Anal. PDE 2 (2009), 83-117.

[55] M.I. Weinstein, Nonlinear Schrödinger equations and sharp interpolation estimates, Comm. Math. Phys. 87 (1983), 567-576.

Université de Versailles St-Quentin and Institut Universitaire de France, LMV

CNRS UMR8100. CurRent address : Ecole polytechnique, CMLS UMR7640 E-mail address: yvan.martel@polytechnique.edu

Université de Cergy Pontoise and Institut des Hautes Études Scientifiques, AGM CNRS UMR8088

E-mail address: merle@math.u-cergy.fr

Université Paul Sabatier and Institut Universitaire de France, imt CNRS UMr 5219. Current address : Université de Nice Sophia-Antipolis, Laboratoire J.A. DieudonnÉ CNRS UMR7351

E-mail address: pierre.raphael@unice.fr 